\title{
DISTORTIONS IN PREDICTED MOTION: PITCH AND DIRECTION INFLUENCE IMAGINED SPEED FOR A VISUAL OBJECT DURING OCCLUSION
}

BY

ADELE CHERISE HOGAN

\begin{abstract}
A thesis
submitted to the Victoria University of Wellington

in fulfilment of the requirements for the degree of

Master of Science in

Cognitive and Behavioural Neuroscience
\end{abstract}

Victoria University of

Wellington 2015 




\begin{abstract}
Visual motion prediction is essential for making key judgements about objects in the environment. These judgements are typically investigated using a time-to-contact (TTC) task, in which an object travels along a straight trajectory and disappears behind an occluder. Participants make a response coinciding with the moment the object would have contacted a visual landmark. The assumption is that the motion continues behind the occluder. This task is used to measure how we perceive and predict the arrival-time of objects. The addition of sound to TTC tasks generally enhances visual judgements. One characteristic which may affect how sound influences visual motion judgements is pitch. A rising pitch is associated with speeded motion and a falling pitch with slowed motion. Pitch change could therefore lead to biases in visual motion judgements; however, this has not yet been investigated. Furthermore, TTC tasks can utilise horizontal or vertical motion. In vertical motion, an additional variable that may be critical for TTC estimations is gravity. It is postulated that humans possess an internal model of gravity that allows us to make accurate predictions for downward motion. This model assumes faster downward than upward motion. However, this model can be wrongfully applied in constant speed tasks, producing faster speed estimations for downward stimuli when there is no acceleration. Therefore, vertical motion could lead to additional biases in visual motion judgements.

This thesis investigated whether pitch and gravity could affect the imagined speed of an object under occlusion. Specifically, a rising pitch was hypothesised to produce speeded predicted motion and falling pitch, slowed predicted motion. I investigated the influence of pitch change in vertical and horizontal planes. I also investigated two different aspects of pitch change, since dynamic pitch is a novel addition to TTC paradigms. Experiment 1A explored gradual pitch change and Experiment 1B used sudden pitch change. The hypothesised pitch effects were observed for a gradual, but not a sudden pitch change. However, a gravity effect was observed across both Experiments $1 \mathrm{~A}$ and $1 \mathrm{~B}$, suggesting the presence of sound does not moderate this effect.

I also examined the cortical substrates of the audio-visual TTC task components by using transcranial magnetic stimulation (TMS) in Experiment 2. The superior temporal sulcus (STS) was targeted in this experiment, as it has been implicated in audio-visual integration. TMS causes neuronal inhibition, and as such, can be used to determine whether an area is involved in a task. If the STS is responsible for audio-
\end{abstract}


visual integration in a TTC task, then TMS to the STS should disrupt the pitch effects evidenced in Experiment 1A. That is, a change in pitch should have no effect on TTC judgements compared to a constant tone. This result was evident only for rising tones, suggesting the involvement of the STS in the generating speeded predicted motion. The pitch effects observed in Experiment 1A and Experiment 2 implicate pitch in the production of biases in motion imagery for visual motion judgements, particularly for visual stimuli under occlusion. 


\section{Acknowledgements}

Firstly, I would like to thank my supervisor, Dr. Gina Grimshaw. Thank you for all the time and energy you have invested in this thesis, and for adopting me graciously into your lab family even though I am not an 'emotional' person. You have been an excellent supervisor and I really appreciate the support and encouragement you provided. I also wish to thank Dr. Steven Prime for supervision in the initial stages of my project, and continuing to provide knowledge as a collaborator from overseas.

Secondly, I would like to thank the members of the CAN lab for providing stimulating conversation, participant numbers, and a general enthusiastic and encouraging environment. Special thanks to Michael Tooley for help with running participants and data queries.

Finally, I would like to thank my office colleagues and friends, Carrie and Catherine, for lending their knowledge, and providing much needed laughter, company, and moral support throughout; and to my family, for always supporting my ambitions and providing me with the means and encouragement to pursue them. 


\section{Table of Contents}

Abstract

Acknowledgements iii

List of Figures vii

List of Tables xi

Introduction 1

Performance in TTC Tasks 2

Spatial Representations of Pitch 4

Pitch-Speed Effects 5

Gravity Effects 6

Cortical Sites of Audio-visual Integration 8

Exploring the Cortical Mechanisms of the STS 10

The Current Experiments 12

Experiment 1A: Gradual Pitch Change at Occluder 14

Method 16

Participants 16

Stimuli and Apparatus 16

Procedure 17

Results and Discussion 18

Horizontal Trials 20

TTC Performance: All Pitch Conditions. 20

TTC Performance: Relative to Silent Baseline. 22

Vertical Trials 26

TTC Performance: All Pitch Conditions. 26

TTC Performance: Relative to Silent Baseline. 28 Summary. 33

Experiment 1B: Sudden Pitch Change at Occluder 34 
Method 35

Participants 35

Stimuli and Apparatus 35

Procedure 35

Results and Discussion 35

Horizontal Trials 35

TTC Performance: All Pitch Conditions. 36

TTC Performance: Relative to Silent Baseline. 38

Vertical Trials 39

TTC Performance: All Pitch Conditions. 39

TTC Performance: Relative to Silent Baseline. 42

Summary. 42

Experiment 2: Influence of TMS to the STS on Predicted Motion 43

Method 45

Participants 45

Stimuli and Apparatus 46

Brain Site Localisation 46

TBS Protocol 49

Procedure 50

Results 52

TTC Performance: All Pitch Conditions 52

Baseline Performance. 52

All TMS conditions. 53

TTC Performance: Relative to Silent Baseline 54

Baseline Performance. 55

All TMS Conditions. 55

General Discussion 57

Interpreting the Behavioural Results 58 
Factors Subserving Successful Integration. 59

Interpreting the TMS Results 61

Localisation of Stimulation Site. 62

Coil Orientation. 65

Motor Threshold Determination. 66

Future Directions 68

Conclusions 70

References 72

Appendix A: Mean (SD) TTC Deviation for Experiment 1A 85

Appendix B: Mean (SD) TTC Deviation for Experiment 1B 87

Appendix C: Mean (SD) TTC Deviation for Experiment 2 89 


\section{List of Figures}

Figure 1. Depiction of a typical lateral TTC task. The visual stimulus (disk) travels horizontally, in a straight line of motion across a screen and moves behind an occluder (grey area). The occluder can be visible and mask-like as in this diagram, or invisible - the stimulus disappears from view at a certain point in time. The participant's task is to indicate when the occluded disk would have reached the landmark. The arrow represents the direction of motion, horizontal dashed lines represent an invisible boundary which contains the stimulus, and the vertical line represents a visual landmark. Diagram is a representation only.

Figure 2. Functional architecture of the superior temporal sulcus by hemisphere, from a meta-analysis of fMRI studies conducted by Hein and Knight (2008). MoPro = motion processing; SpeechPro = speech processing; $\mathrm{ToM}=$ theory of mind; $\mathrm{AV}$ $=$ audio-visual integration; and FacePro $=$ face processing .

Figure 3. Example of A) a typical horizontal trial; and B) a typical vertical trial (not to scale). Upon key press, the visual stimulus (the white disc) moved along the track towards the black rectangle and became occluded from view. Participants had to make another key press when they estimated the visual stimulus to have reached the white line. Sequence of events:

1. Spacebar initiates stimulus movement; at the same time a $500 \mathrm{~Hz}$ tone is played.

2. Visual stimulus moves along a constant trajectory, until key press. Pitch stays the same or rises/fall in pitch by $21 \mathrm{~Hz} / \mathrm{s}$.

3. ISI for $0.5 \mathrm{~s}$.

4. New randomised trial appears.

Figure 4. Deviations in TTC estimates for visual stimuli for constant, rising, falling and silent conditions as a function of stimulus speed for horizontal trials in Experiment 1A. The error bars represent the standard error.

Figure 5. Deviations in TTC estimates for visual stimuli for slow, medium and fast speeds as a function of landmark position for horizontal trials in Experiment 1A. The error bars represent the standard error. 22

Figure 6. Deviations in TTC estimates for visual stimuli for constant, rising, falling and silent conditions as a function of landmark position for horizontal trials in Experiment 1A. The error bars represent the standard error. 
Figure 7. Relative deviations in TTC estimates for visual stimuli for constant, rising, and falling conditions as a function of stimulus speed for horizontal trials in Experiment 1A. The error bars represent the standard error.

Figure 8. Relative deviations in TTC estimates for visual stimuli for constant, rising, and falling conditions as a function of landmark for horizontal trials in Experiment 1A. The error bars represent the standard error.

Figure 9. Deviations in TTC for visual stimuli at near, middle and far landmark positions for upward and downward stimulus motion in Experiment 1A for vertical trials. Error bars represent the standard error.

Figure 10. Relative deviations in TTC estimates for visual stimuli in the constant, rising, falling and silent conditions at slow, medium and fast stimulus speeds, for upward and downward motion in Experiment 1A for vertical trials. The error bars represent the standard error.

Figure 11. Deviations in TTC for visual stimuli in constant, rising, falling and silent trials by landmark position at slow, medium and fast stimulus speeds in Experiment 1A for vertical trials. Error bars represent the standard error. 28

Figure 12. Relative deviations in TTC for visual stimuli in constant, rising, and falling trials by stimulus speed for upward and downward movement in Experiment $1 \mathrm{~A}$ for vertical trials. Error bars represent the standard error.

Figure 13. Relative deviation in TTC estimates for visual stimuli in the constant, rising and falling pitch conditions by landmark position at slow, medium and fast speeds in Experiment 1A for vertical trials. Error bars represent the standard error.

Figure 14. Relative deviation in TTC estimates for visual stimuli in the constant, rising and falling pitch conditions by landmark position for upward and downward motion in Experiment 1A for vertical trials. Error bars represent the standard error.

Figure 15. Deviations in TTC estimates for visual stimuli in slow, medium and fast speed conditions at the near, middle and far landmarks in Experiment 1B for horizontal trials. Error bars represent the standard error.

Figure 16. Deviations in TTC for visual stimuli by rightward or leftward motion, at near, middle and far landmark positions in Experiment 1B for horizontal trials. Error bars represent the standard error. 
Figure 17. Relative deviations in TTC for visual stimuli in constant, rising and falling pitch conditions by near, middle and far landmark positions in Experiment 1B for horizontal trials. Error bars represent the standard error.

Figure 18. Deviation in TTC for upward and downward moving stimuli at the near, middle and far landmark positions in Experiment 1B for vertical trials. Error bars represent the standard error. 40

Figure 19. Deviations in TTC for visual stimuli in the constant, rising, falling, and silent conditions at slow, medium and fast stimulus speeds in Experiment 1B for vertical trials. Error bars represent the standard error.

Figure 20. Deviations in TTC for visual stimuli in slow, medium and fast speed conditions at the near, middle and far landmarks in Experiment $1 \mathrm{~B}$ for vertical trials. Error bars represent the standard error.

Figure 21. Lateral view of the cerebrum showing the left superior temporal sulcus (in blue). Adapted from Bear, Connors and Paradiso (2006).

Figure 22. Trial procedure in Experiment 2. White dot flashes green briefly before moving in a straight line of motion behind the occluder. Participants press the spacebar key when they estimate the white dot has reached the landmark (white line). Stimuli are not to scale.

Sequence of events:

1. White dot waits at one end of a track for $0.5 \mathrm{~s}$.

2. Dot flashes green for $0.5 \mathrm{~s}$ to signal trial is about to start.

3. After $0.5 \mathrm{~s}$, white dot moves to opposite end of track. $500 \mathrm{~Hz}$ tone paired with dot, changes at occluder: no change/ rising or falling by $18 \mathrm{~Hz} / \mathrm{s}$.

4. Visual mask presented for $0.5 \mathrm{~s}$.

5. New randomised trial appears.

Figure 23. Mean TTC deviation for constant, rising, falling and silent trials in the no TMS condition for upward and downward stimuli for A) Experiment 2; and B) Experiment 1A (Values are for the slow stimulus speed and far landmark position only). Error bars reflect the standard error of the mean. 53

Figure 24. Deviations in TTC estimation for visual stimuli in the constant, rising, falling and silent pitch conditions by TMS condition. Error bars reflect the standard error. 
Figure 25. Relative TTC deviation for constant, rising, and falling and trials in the no TMS condition for upward and downward stimuli, relative to silent trials for A) Experiment 2; and B) Experiment 1A (Values are for the slow stimulus speed and far landmark position only). Error bars reflect the standard error of the mean.

Figure 26. Relative deviations in TTC estimation for visual stimuli in the constant, rising and falling pitch conditions by TMS condition. Error bars reflect the standard error. 


\section{List of Tables}

Table 1. Actual TTC of visual stimulus (in seconds) as a function of landmark position and stimulus speed for experiments $1 A$ and $1 B$.

Table 2. Original Talairach coordinates from Hein and Knight (2008), transformed into MNI coordinates for Experiment 2.

Table 3. MNI coordinates by participant $(\mathrm{mm})$ for the two target sites: the left and right STS, and motor threshold (MT) and intensity of TBS stimulation in Experiment 2. 

Distortions in Predicted Motion: Pitch and Direction Influence Imagined Speed for a Visual Object during Occlusion

In our day-to-day lives, we receive a constant stream of information from the environment that we use to make judgements. One judgement we are required to make regularly is the time it will take for a moving object to reach us. This judgement is useful in everyday activities such as catching a ball or navigating a busy road. If we take a road scene for example, a moving car is defined by visual and auditory spatialtemporal information. This information is useful in determining the cars current location and predicting how long it will take to reach a new location. These computations are essential to determine whether we can enter traffic while driving, or cross the street as a pedestrian.

One factor that influences our prediction of an object's future location is obviously its speed (Wuerger, Meyer, Hofbauer, Zetzsche, \& Shill, 2010; Battaglini, Campana, \& Casco, 2013). In addition, other factors such as accompanying sound or the object's direction can also be integrated into that judgement (Gordon \& Rosenblum, 2005; Hofbauer, Wuerger, Meyer, Roehrbein, Schill, \& Zetzsche, 2004; Hubbard \& Courtney, 2009; Wuerger et al., 2010; Zago, Iosa, Maffei, \& Lacquaniti, 2010; Zago \& Lacquaniti, 2005a). In this thesis, I will explore how the auditory pitch of a moving object and its direction of travel can influence predictions about its movement. I will further explore the neural mechanisms that allow us to integrate information from these different modalities into our judgements about moving objects.

An experimental paradigm that is used to gauge how well observers are able to perceive and predict visual motion is the prediction-motion task (Tresilian, 1995; see Figure 1). In a typical prediction-motion task, a visual stimulus moves along an assigned trajectory and becomes occluded from view at a certain time-point. Participants make a response coinciding with the moment the stimulus would have reached a visual landmark, assuming motion continues behind the occluder. This temporal estimation is often labelled time-to-contact (TTC; Lee, 1976; also termed time-to-arrival, time-to-collision and time-to-passage). Methods other than TTC can be used in prediction-motion tasks (for example, relative judgements; Tresilian, 1995), however TTC paradigms are the primary method of investigation and main focus of this thesis. 


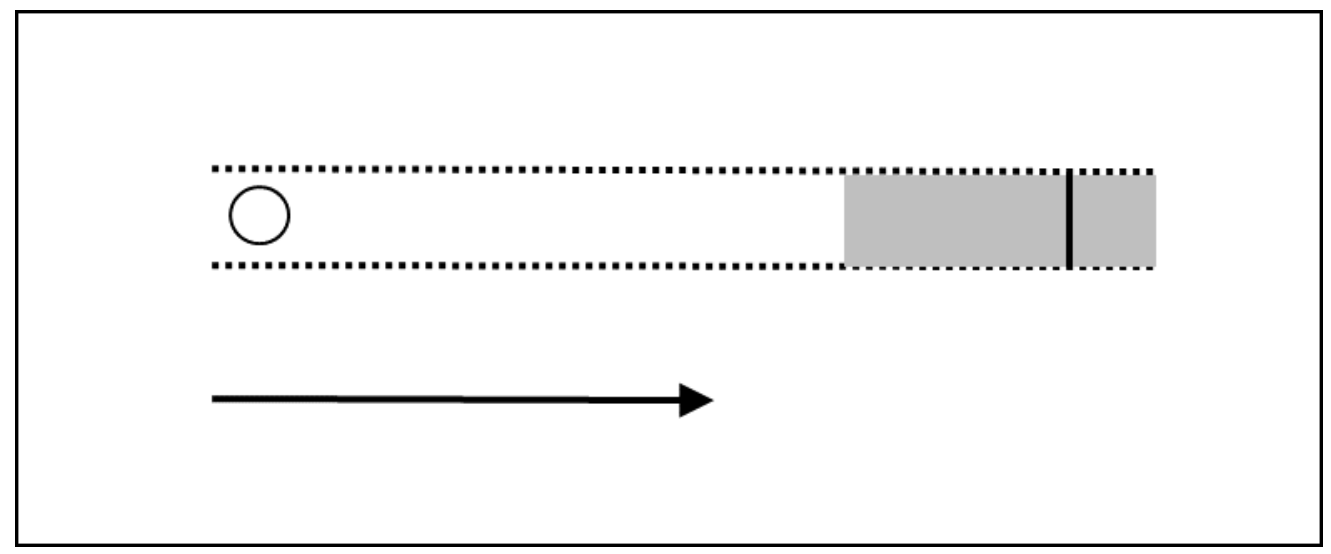

Figure 1. Depiction of a typical lateral TTC task. The visual stimulus (disk) travels horizontally, in a straight line of motion across a screen and moves behind an occluder (grey area). The occluder itself can be visible and mask-like as in this diagram, or invisible: the stimulus simply disappears from view at a certain point in time. The participant's task is to indicate when the occluded disk would have reached the landmark. The arrow represents the direction of motion, horizontal dashed lines represent an invisible boundary which bounds the stimulus trajectory, and the vertical line represents a visual landmark. Diagram is a representation only.

\section{Performance in TTC Tasks}

A variety of manipulations have been explored in TTC paradigms, such as varied stimulus speeds, viewing times, occlusion times, and landmark positions (DeLucia \& Liddell, 1998; Gray \& Thornton, 2001; Peterken, Brown, \& Bowman, 1991; Schiff \& Oldak, 1990; Zago et al., 2010). These methodological manipulations have elicited contradictory results; some indicating humans underestimate in TTC situations (Gordon \& Rosenblum, 2005; Gray \& Regan, 1998), while others show overestimations (Gray \& Regan, 2004; Renolds, 1968). An underestimation occurs when the stimulus is perceived as moving faster than it actually is, eliciting a premature TTC response (i.e., the stimulus would not yet have reached the landmark). Conversely, an overestimation occurs when the visual stimulus is judged as moving slower than it actually is, producing a delayed TTC response (i.e., the stimulus would have overshot the visual landmark by the time the response is made). In this review, I will focus on four factors affecting TTC estimates: (a) stimulus speed, (b) visual occlusion, (c) stimulus direction, and (d) the addition of sound.

Firstly, the actual stimulus speed affects TTC estimates; a stimulus which travels too fast or slow results in inaccurate estimates (Wuerger et al., 2010). The 
former usually produces underestimations, and the latter produces overestimations (Battaglini et al., 2013; Wuerger et al., 2010).

Secondly, the stimulus occlusion period also influences TTC estimates. In TTC paradigms, the occlusion period is arguably the key phase, as it is during this stage that mental imagery becomes important (Battaglini et al., 2013). Information about stimulus velocity is extrapolated when the stimulus is visible, and stored in working memory to be retrieved during occlusion (Battaglini et al., 2013; Kaas, Weigelt, Roebroeck, Kohler, \& Muckli, 2010). Both the stimulus viewing time-amount of time the stimulus is visible prior to occlusion-and occlusion time itself affect TTC estimates (DeLucia, Kaiser, Bush, Meyer, \& Sweet, 2003). TTC accuracy increases slightly with increasing viewing time, as more time is provided for speed extrapolations (Gordon \& Rosenblum, 2005). However, the occlusion period has the most influence; accuracy decreases with increasing occlusion time due to memory decay (Gordon \& Rosenblum, 2005; Peterken et al., 1991). This information suggests that it becomes increasingly difficult to maintain the correct stimulus speed using mental imagery as time elapses.

Thirdly, TTC estimations are influenced by the direction of stimulus motion (Zago et al., 2010). TTC paradigms can involve lateral (allocentric) or looming (egocentric) visual motion (Calabro, Beardsley, \& Vaina, 2011; Coull, Vidal, Goulon, Nazarian, \& Craig, 2008). For the sake of simplicity, unless otherwise specified, all subsequent TTC tasks referred to in this thesis will involve lateral motion. Furthermore, typical TTC tasks utilise horizontal motion (Gray \& Thornton, 2001; Hancock \& Manster, 1997; Peterken et al., 1991; Reynolds, 1968; Wuerger et al., 2010); however performance has also been investigated using vertical motion (Hubbard \& Courtney, 2009; Zago \& Lacquaniti, 2005a; Zago \& Lacquaniti, 2005b). In comparison to horizontal motion, vertical motion can complicate estimates, as downward motion produces speeded predicted motion due to gravity (Zago \& Lacquaniti, 2005a).

Finally, sound also influences TTC judgements. One might assume observers would use a combination of auditory and visual motion cues to produce a reliable prediction about a moving object in the real world. However, it is only in the last decade that researchers have begun to use a combination of visual and auditory stimuli in TTC tasks (Gordon \& Rosenblum, 2005; Hofbauer et al., 2004; Wuerger et al., 2010). This combination typically improves TTC accuracy (Gordon \& Rosenblum, 2005; Hofbauer et al., 2004; Wuerger et al., 2010). However, audio-visual TTC tasks have only investigated whether TTC performance is improved with a temporally 
congruent sound (Gordon \& Rosenblum, 2005; Hofbauer et al., 2004; Wuerger et al., 2010); as of yet, no bimodal TTC task has investigated how sound can distort visual motion estimates.

I have introduced four factors which influence TTC estimates in predictionmotion tasks: stimulus speed, visual occlusion, stimulus direction and the addition of sound. In this thesis, I focus on stimulus direction and sound as factors that are integrated into our judgements about moving objects. Specifically, I consider how knowledge of physics (related to gravity) and the changing pitch that accompanies a moving object can alter TTC judgements. In Experiment 1, I use behavioural methods to determine how sound and direction can affect predicted motion judgements, and in Experiment 2 I use transcranial magnetic stimulation (TMS) to further our understanding of the neural systems that support such integration. First, I outline how a particular characteristic of sound - pitch — could affect motion imagery due to multilevel relationships with other variables. Second, I introduce stimulus direction as an additional factor in producing altered TTC judgements.

\section{Spatial Representations of Pitch}

One important aspect of any sound is pitch, the psychological correlate of frequency (Stevens, Volkmann, \& Newman, 1937). However, pitch is not just about frequency, it also has crossmodal representations in space (Hidaka, Teramoto, Keetels, \& Vroomen, 2013). These spatial mappings are evident in the Spatial-Musical Association of Response Codes effect, in which responses for high-pitched tones are faster when using the 'up' key in speeded reaction tasks, and responses for low-pitched tones are faster when using the 'down' key (Rusconi, Kwan, Giordano, Umilta, \& Butterworth, 2006). Furthermore, people are faster to identify shapes that appear high in the visual field after a high-pitched tone, and faster to identify shapes low in the visual field following a low-pitched tone (Godfrey, 2011). In spatial cueing tasks such as these, the magnitude of the cueing effect depends on the pitch difference; larger effects are found for larger pitch differences between tones (Chiou \& Rich, 2012). These mappings can move beyond simple tones, as ascending pitch can direct attention to the upper visual field and descending pitch to the lower visual field (Takeshima \& Gyoba, 2013).

It is evident pitch exerts vertical spatial mappings, but evidence for horizontal mappings are less established. There is evidence to suggest pitch is mapped horizontally in musicians; specifically, left spatial mappings for low-pitched tones, and right for 
high-pitched tones (Lidji, Kolinsky, Lochy, \& Morais, 2007). This pitch mapping reflects the tonal layout of keyboard instruments such as a piano. In non-musicians, in order to achieve the same mappings, a task needs to be used in which pitch is explicitly processed (Chiou \& Rich, 2012; Lidji et al., 2007). Spatial mappings are important to keep in mind when using sound in TTC tasks, as the pitch could influence the results by directing attention to different visual-field locations.

\section{Pitch-Speed Effects}

Pitch is also associated with speed. Evidence for this relationship comes from a real-world example - The Doppler Effect - in which the sound produced by a constant moving object drops in frequency as it approaches and recedes from a stationary viewer (Doppler; as cited in McBeath \& Neuhoff, 2002). Furthermore, the magnitude of the frequency drop depends on the speed of the sound-source (McBeath \& Neuhoff, 2002). However, many people experience a rise in frequency as the sound approaches (Neuhoff \& McBeath, 1996). This perceptual experience is termed The Doppler Illusion, as there is no actual frequency rise. The illusion suggests that people hold a preconceived notion that objects approaching at speed are accompanied by a rise in pitch. However, there is no evidence for a bidirectional relationship; it is unknown whether increasing the pitch of a sound can speed up the predicted motion of a moving object in situations in which the speed actually remains constant. This is an area that warrants investigating. If pitch can alter speed estimates in visual motion, it would suggest multisensory integration - integration of information from both senses in parallel—of the auditory pitch and visual information to form a distortion in motion imagery (Schmiedchen et al., 2012).

Another reason for a possible inherent relationship between pitch and speed is that high-pitched tones are perceptually 'faster' than low-pitched tones. Collier and Hubbard (1998) had participants rate sequences of tones differing in pitch (261.6, 523.2, and 1046.4 Hz) and tempo (slow, medium, or fast) by how fast they sounded, using Likert scales. Of particular interest, people rated high-pitched sequences of tones as faster and speeding up more than low-pitched sequences; and ascending sequences of tones as faster than descending sequences.

However, this relationship between pitch and speed may not be symmetrical. Eitan and Granot (2004) asked participants to visualise the movement of a cartoon figure while listening to melodies with different pitch properties. A rise in pitch was associated with an increase in the speed of the cartoon, however a decrease in pitch was 
not associated with a decrease in speed. Even children associate a rise in pitch with an increase in speed, but do not associate a drop in pitch with decreasing speed (Eitan \& Tubul, 2010). With regards to the current study, it is not clear how changes in pitch might affect estimates of the speed of objects. Sound in an unchanging context has been shown to affect the predicted speed in a visual apparent motion task (Manabe \& Riquimaroux, 2000). However, no studies have investigated how dynamic changes in pitch could lead to biases in actual visual motion judgements.

\section{Gravity Effects}

In addition to pitch, biases in TTC estimates can be produced by the direction of visual motion. TTC tasks can investigate motion in the horizontal or vertical plane. Vertical motion introduces a new factor to consider: gravity. It is postulated that humans have an internal model of gravity which we use to estimate when a falling object will reach the ground (Zago \& Lacquaniti, 2005b). This model assumes the laws of gravity; that downward travelling objects will accelerate, and therefore travel faster than objects with upward motion (McIntyre, Zago, Bethoz, \& Lacquaniti, 2001). There is evidence to suggest this model is invoked when performing vertical TTC tasks, even though the object itself appears as a computer generation, and is not subject to gravity (McIntyre et al., 2001; Zago et al., 2010; Zago \& Lacquaniti, 2005a; Zago \& Lacquaniti, 2005b; Zago, McIntyre, Senot, \& Lacquaniti, 2008). As a result, TTC estimates are fairly accurate when visual stimuli ascend or descend under the laws of gravity, but become increasingly biased when motion does not adhere to these rules (Zago et al., 2010).

The gravity simulation can be invoked even in the absence of gravity. McIntyre et al. (2001) conducted a TTC task in which astronauts caught a downward moving ball in a space shuttle under zero-gravity, or while still on earth under normal gravitational conditions. The ball could move at three initial speeds $(0.7,1.7$ and $2.7 \mathrm{~m} / \mathrm{s})$, and electromyography (EMG) recordings were used as an index of muscle activity in the bicep. Performance was fairly accurate (within 200ms) on earth under normal gravitational conditions. However, in zero-gravity, EMG recordings showed an anticipatory response to the ball in line with the predicted response in a gravity-present environment, suggesting humans expect downward moving objects to accelerate under gravitational laws.

Furthermore, the invocation of the gravity model may act outside conscious control. Evidence for unconscious invocation of the internal model is presented in an 
experiment conducted by Zago and Lacquaniti (2005a). These authors investigated whether there are multiple internal models to suit performance in different environments, or whether one internal model is adapted to fit the situation. A visual target moved downward on a screen, and disappeared from view prior to reaching the bottom. At the same time, a rubber ball was dropped behind the screen; its velocity was controlled by an electromagnet to maintain synchronous position with the virtual target. Participants punched the previously hidden ball when it dropped from below the screen. Starting target speeds were varied and targets could descend under gravity, or move at constant descent (zero-gravity). Performance was accurate in gravity-present trials, but participants responded prematurely when the object moved at a constant speed.

Furthermore, accuracy improved with training but remained impaired in comparison to gravity-present trials. This outcome suggests there is one internal model for gravitybound objects which is adapted to fit performance for objects unbound by gravity.

Finally, vertical stimulus motion was investigated by Hubbard and Courtney (2009), with an important addition: sound. The study of Hubbard and Courtney reflects the integration of gravity, pitch and speed effects, using a prediction-motion task. Two blocks of trials were presented in which a visual stimulus moved vertically (upward or downward), paired with a tone which could rise or fall in pitch. Furthermore, stimulus pairings could be congruent—stimulus moved downward and tone fell in pitch—or incongruent; stimulus moved downward and tone rose in pitch. In one block of trials, visual displacement was probed, and the other probed auditory displacement. Instead of using TTC estimates, the authors probed the final spatial location of the visual stimulus, or the final pitch for the auditory stimulus. There was no occlusion period in this task. Participants indicated whether the probe was presented at the same, or a different location to the final inducing stimulus, and determined whether the direction of motion in the other stimulus modality was ascending or descending. For visual stimuli, a same response meant the probe appeared in the same location as the final position of the visual stimulus and for auditory stimulus a same response indicated the tone played was of the same pitch as the final segment of the sound (opposed to a higher or lower tone). Overestimations were largest for visual stimuli accompanied by downward motion in the visual probe block, and auditory stimuli which fell in pitch in the auditory probe block. In this situation, an overestimation means participants were more likely to give a 'same' response when the probe location or sound was more advanced than the target. Therefore, an overestimation indicated the stimuli were judged as moving faster, as they 
reached a further location in the same amount of time. These effects were also additive, as the largest overestimations were evidenced for downward movement combined with a falling pitch. The results of this study suggest an effect of congruency; if pitch and stimulus direction are congruent, predicted motion is faster. However, the congruent combination including downward motion speeds predicted motion more than the combination including upward motion.

Overestimations for visual stimuli in Hubbard and Courtney (2009) provide support for an effect of gravity in prediction-motion tasks. However, this gravity effect is hard to quantify without a horizontal control condition. How pitch affects horizontal motion estimates compared to vertical is critical in interpreting spatial mappings and the magnitude of the gravity effect. The pitch-speed relationship denotes a rising pitch should speed motion estimates and a falling pitch should slow motion estimates. Contradictory to predictions laid out by the pitch-speed relationship, overestimations were evidenced for falling-pitched, but not rising-pitched tones. These results present interesting connotations for pitch versus gravity effects, and how these are integrated; the specifics of which need more exploration.

\section{Cortical Sites of Audio-visual Integration}

Thus far, the focus of this review has been on possible relations between visual motion, gravity, pitch and speed from a purely behavioural standpoint. However, multisensory integration can be better understood by identifying neural mechansims that underpin it. Neuroimaging techniques can be used to determine audio-visual integration areas in the brain by determining areas that are most active when both auditory and visual information are present (Beauchamp, 2005). Specifically, functional magnetic resonance imaging (fMRI) can detect changes in the blood oxygen level in the brain at a voxel by voxel basis, detecting and mapping changes related to neuronal activity (Logothetis \& Pfeuffer, 2004). This method allows for a strong correlational relationship between brain function and cognition. Therefore, if an area is activated by bimodal stimuli, then these sites may play a key role in integration. A way to quantify this activation is by attaining a superadditive response during an audio-visual task. Superadditivity occurs when the neuronal output is greater for bimodal stimuli than for the combined signals of either modality alone; in this case, there is a stronger signal for audio-visual stimuli than the combined responses for auditory and visual modalities alone (Calvert, Hansen, Iversen, \& Brammer, 2001). We can then directly stimulate those same regions to determine how they causally affect motion prediction. 
Superadditive responses in the blood oxygen level dependent (BOLD) signal for temporally congruent stimuli during an audio-visual task have been shown in several brain regions using fMRI (Calvert et al., 2001). These regions include the superior colliculi, right inferior frontal gyrus, right lateral sulcus, ventromedial frontal gyrus, insula, right superior parietal lobule, right inferior parietal sulcus, left superior occipital gyrus and left superior temporal sulcus (Calvert et al., 2001). The likelihood of each of these regions in binding audio-visual information in a TTC can be determined by systematically investigating the type of stimuli preferentially processed by these regions.

Parietal regions such as the intraparietal sulcus deal with the binding of mostly visuo-tactile information (Saito, Okada, Morita, Yonekura, \& Sadato, 2003). Lateral occipital areas preferentially process shape specific visual and tactile information (Beauchamp, 2005). In addition, a multitude of frontal regions-including the right inferior frontal gyrus - are responsible for audio-visual integration (Chikazoe, Konishi, Asari, Jimura, \& Miyashita, 2007).

The intraparietal sulcus plays a critical role in detecting spatially correspondent multisensory stimuli (Ghazanfar \& Schroeder, 2006). As such, the intraparietal sulcus may not be a key area in subserving audio-visual integration in a TTC task that focuses on auditory manipulations, as visual information is thought to provide more weighting in spatial aspects of tasks (Welch \& Warren, 1980). An example of spatial dominance for vision over audition in the behavioural domain is present in a study in which baby barn owls were fitted with ocular prisms; this created visual displacement by $11^{\circ}, 23^{\circ}$, or $34^{\circ}$, while leaving auditory localisation intact (Knudsen \& Knudsen, 1989). Baby owls were exposed to noise bursts, or a light emitting diode, while speed and accuracy of orienting to the stimuli was recorded. The prisms caused not only visual, but also auditory displacement; there was a shift in the localisation of the sound in line with the spatial location of the visual stimulus. Additionally, when prisms were removed after 102 or 103 days of training, owls oriented their head to the visual source perfectly, but continued to orient to the right of the sound (by $\sim 8^{\circ}$ for $11^{\circ}$ prisms, $\sim 18^{\circ}$ for $23^{\circ}$ prisms, and $\sim 14^{\circ}$ for $34^{\circ}$ prisms). These results demonstrate when vision and audition are spatially disparate, visual cues prevail over auditory cues.

The superior temporal sulcus deals with temporally specific information related to events (Ghazanfar \& Schroeder, 2006). For example, a flash of light accompanied by two or more beeps is perceived as multiple flashes of light (Shams et al., 2002). 
However, these illusory flashes are only observed when the beeps are presented within $100 \mathrm{~ms}$ of the flash (Shams et al., 2002), demonstrating the importance of temporal correspondence in tasks with auditory manipulations. Given that audition dominates temporal aspects of crossmodal tasks (Shams et al., 2002; Wada, Kitagawa, \& Noguchi, 2003; Watanabe \& Shimojo, 2001), pitch manipulations to a TTC task would rely on temporally, rather than spatially correspondent stimuli. Therefore, the superior temporal sulcus (STS) seems to be an area that could likely be involved in integrating the auditory and visual dimensions of the task. There has been a lot of interest in the STS in the last decade; it has been shown to be implicated in not only audio-visual integration, but also diverse functions such as theory of mind, biological motion, speech processing and face processing (Allison, Puce \& McCarthy, 2000; Grossman, Battelli, \& PascualLeone, 2005; Hein \& Knight, 2008; McGurk \& MacDonald, 1976; Saygin, 2007; Watson et al., 2014, Werner \& Noppeney, 2009).

\section{Exploring the Cortical Mechanisms of the STS}

In a meta-analysis using fMRI, Hein and Knight (2008) mapped areas of activation in the STS to identify five functional domains: theory of mind, audio-visual processing, motion processing, speech processing and face processing (shown in Figure 2). Of particular interest in this thesis is that, in both hemispheres, audio-visual integration seems to be localised to the posterior region of the STS. This meta-analysis reveals that although there is general clustering of activation points for studies within the same category,- - audio-visual sites clustered together and speech processing sites

clustered together - there is still a lot of variability in the locus of each category within the STS. Specifically, there are audio-visual activation points spanning a large section of the STS.

One reason for the lack of specificity in sites targeted in audio-visual studies is the high variability in tasks and stimuli. For example, Beauchamp, Lee, Argall, and Martin (2004) used fMRI to determine whether the posterior STS is involved in integrating information about complex audio-visual stimuli. Participants were presented with pictures of man-made objects, such as a hammer, or an animal, such as a cat. Auditory stimuli were the sounds associated with the use of that tool, or sounds the animal could make, such as "bang, bang" and "meow" respectively. Stimulus sets also included meaningless stimuli such as scrambled pictures, or meaningless sounds, such as ripple sounds. Pictures and sounds could be paired so that a semantically meaningful or meaningless combination was produced, and in some trials pictures or sounds were 
presented in isolation. Results showed an enhanced BOLD signal in the STS for meaningful combinations compared to combinations that were semantically incongruent and devoid of meaning. In addition, there was an increased BOLD signal for combined auditory and visual stimuli compared to unimodal stimuli, suggesting the STS preferentially processes audio-visual information.

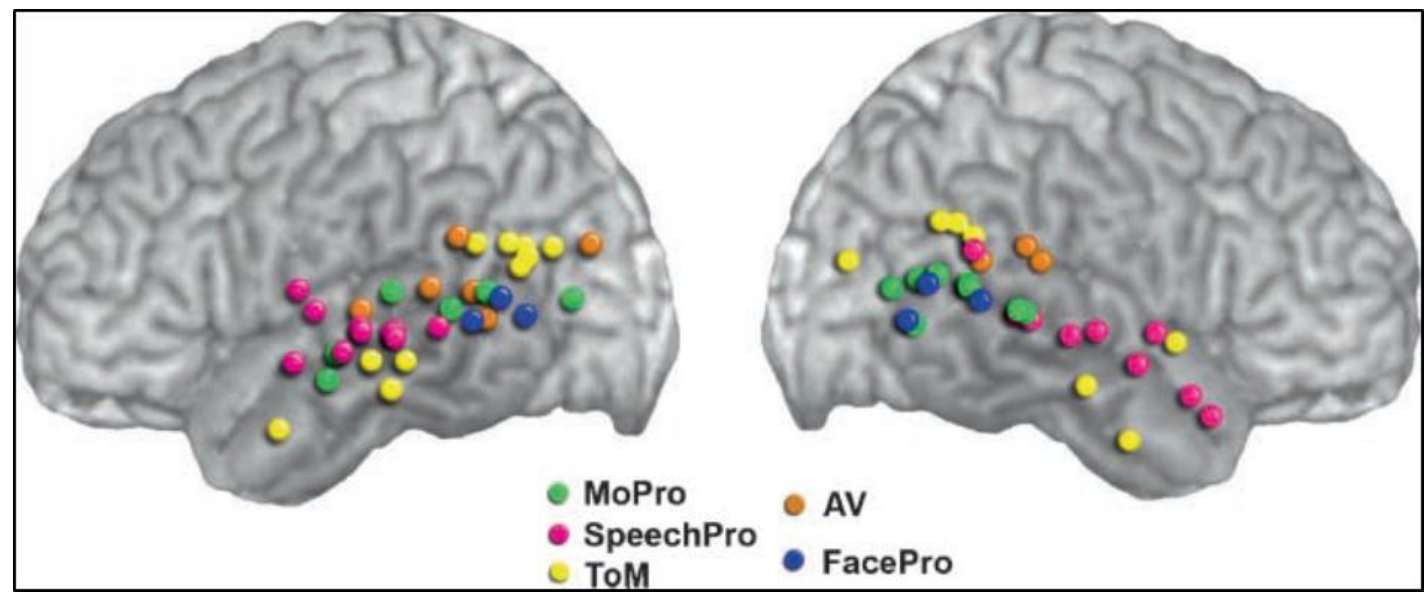

Figure 2. Functional architecture of the superior temporal sulcus by hemisphere, from a meta-analysis of fMRI studies conducted by Hein and Knight (2008). MoPro = motion processing; SpeechPro = speech processing; ToM = theory of mind; AV = audio-visual integration; and FacePro = face processing.

In comparison, Noesselt et al. (2007) also used an audio-visual fMRI experiment, but with a very different task. Participants fixated on a central point, while irrelevant visual stimuli were presented in the corner of the screen (either a red cross or a green square). During audio-visual trials, a sound burst was played along with the presentation of the visual stimulus. In these trials, the auditory and visual information could be presented simultaneously, or at different time-points (100ms disparity). At random time intervals, the central fixation would brighten, signalling a response was required. An enhanced BOLD signal was observed in the STS when irrelevant stimuli were audio-visual and temporally correspondent compared to unimodal stimuli. Furthermore, a reduced BOLD signal was found for audio-visual stimuli that were presented out of synchrony.

Both Noesselt et al. (2007) and Beauchamp et al. (2004) found increased signalling for stimuli that were semantically or temporally congruent, suggesting a role for the STS in binding auditory and visual signals from a single event. In addition, Noesselt et al. and Beauchamp et al. demonstrated similar neural outcomes despite vastly different tasks and stimuli. However, similar neural outcomes does not necessarily mean the STS is primarily responsible for the behavioural effects. Despite 
having good spatial and temporal resolution, fMRI is only able to detect areas involved in a task, which do not necessarily determine if these areas are crucial to the task (Logothetis \& Pfeuffer, 2004). FMRI relies on the assumption that there is a relationship between cerebral blood flow and brain activity, and as such this technique cannot be used to infer causal relationships between brain regions (Logothetis \& Pfeuffer, 2004).

A technique that can be used to infer causation is transcranial magnetic stimulation (TMS; Sandrini, Umilta, \& Rusconi, 2011). TMS operates on the principle of electromagnetic induction whereby the TMS coil generates a brief electric current which in turn generates a magnetic field, fluctuating in strength (Pascual-Leone, Walsh, \& Rothwell, 2000). The magnetic field creates a current in the cortical area of stimulation, which interferes with local neuronal activity (Walsh \& Cowey, 2000). This interference is created by the induction of neural noise into the cortex below, sending the neurons into a state of disorder (Walsh \& Cowey, 2000). Therefore, if TMS to a given area causes a disruption in task performance, the area can be deemed necessary for the task. This technique could be used to answer the mounting questions about how pitch and direction affect performance in TTC paradigms and the neuroanatomical substrates of the integration of auditory and gravity-based effects into motion judgements. TMS can also be used to determine whether integration sites are specific to a particular modality. For example, through dissociations it may be possible to separate the effects of pitch and gravity. Given that the STS is primarily an audio-visual integration site, disruptions to the STS might be expected to disrupt pitch effects, but not gravity effects.

\section{The Current Experiments}

It is known that sound can affect judgements about the motion of an object (Prime \& Harris, 2010; Teramoto, Hidaka, Gyoba, \& Suzuki, 2010; and Wuerger et al., 2010). Previous audio-visual TTC tasks have found concurrent sound generally enhances visual TTC performance (Gordon \& Rosenblum, 2005; Hofbauer et al., 2004; Wuerger et al., 2010). However, no studies have dynamically altered the pitch of an accompanying sound to determine whether changes in pitch produce changes in predicted motion. Given the relationship between pitch and speed (see, The Doppler Effect; Doppler; as cited in McBeath \& Neuhoff, 2002), it is possible pitch could produce biases in speed estimates in a TTC task. There is a gap in the current literature for determining how pitch affects TTC estimates; this thesis targets this gap. When 
sound is added to visual motion, the pitch becomes important. Pitch has been shown to express vertical spatial mappings (Chiou \& Rich, 2012; Godfrey, 2011), although horizontal mappings are not well established (Chiou \& Rich, 2012; Lidji et al., 2007). Furthermore, investigations on pitch affecting visual motion estimates have only been addressed using vertical prediction-motion (Hubbard \& Courtney, 2009). Literature on downward vertical motion has demonstrated that gravity affects visual motion estimates, even if stimuli are not subject to gravity (McIntyre et al., 2001; Zago et al., 2010; Zago \& Lacquaniti, 2005a).

In order to address the lack of literature examining pitch effects in TTC paradigms, this thesis investigated the effects of pitch on motion estimates, using horizontal and vertical motion to test the effects of direction of movement. A TTC task was devised in which a visual stimulus moved at a constant speed within a narrow track. Speed was kept constant, because acceleration has been shown to alter TTC judgements (Bootsma \& Oudejans, 1993), and also because the aim was to determine how pitch affected predictions for a stimulus travelling at a constant speed. The stimulus moved within a track of the same width, as pitch has been shown to produce vertical displacement, even in horizontal prediction-motion tasks (see Experiment 2 in Hubbard \& Courtney, 2009). Due to the thin existing literature in the area, I also manipulated the landmark position and the visual stimulus speed to see how these parameters might interact with the pitch and direction manipulations. By this reasoning, Experiments 1A and 1B were exploratory in nature, with the aim of describing the effect of pitch and direction on motion prediction, and identifying the parameters at which these effects were strongest. Three landmark positions were used, as well as three different stimulus velocities.

In addition, as few studies exist which pair visual motion with pitch change, the behavioural experiments were designed to assess the circumstances under which pitch would bias TTC estimates. To this end, two pitch manipulations were examined in separate experiments. In Experiment 1A, the pitch change was gradual (ascending or descending) and began at the transition point where the stimulus became occluded. In Experiment 1B, the pitch change was sudden (a jump in pitch compared to a steady rise or fall). Two control conditions were also included. In one, the moving stimulus was accompanied by a constant pitch, and in the other, the moving stimulus appeared without sound (silent condition). 
The aim of Experiment 1 was to identify the optimal experimental parameters that captured both pitch and gravity effects. Those parameters were then used in Experiment 2, in a pilot study in which I used TMS to explore the role of the STS in the integration of pitch and gravity with visual motion. The STS is an area recognised as being involved in different audio-visual tasks (Calvert et al., 2001; Beauchamp, Nath, \& Pasalar, 2010; Hocking \& Price, 2008). However, no studies have investigated whether the STS is responsible for integration in TTC tasks, and consequently, no research has been conducted into the cortical loci of TTC task performance. A logical way to target this gap would be to examine task performance following inhibition of neuronal activity in the STS with TMS. Multiple TMS paradigms have produced impaired performance in other audio-visual tasks when targeting the STS (Calvert et al., 2001; Beauchamp et al., 2010; Hocking \& Price, 2008), making it a viable target site. Experiment 2 was conducted to examine whether the STS is responsible for this binding. If the STS is responsible for binding the auditory and visual components of the moving stimulus, then inhibitory TMS to this site will disrupt the audio-visual binding process.

Disruption would be evident by no effect of pitch on visual motion judgements, reflecting performance during constant and silent conditions. However, TMS was not expected to affect any gravity-induced effects, so the gravity effect should remain. TMS was applied to the right and left posterior STS, and compared to performance in two control conditions; TMS to the vertex and no TMS.

\section{Experiment 1A: Gradual Pitch Change at Occluder}

Experiment $1 \mathrm{~A}$ was conducted to elucidate the role pitch plays in visual motion judgements in a TTC task, as no previous studies have examined how a pitch manipulation can affect these judgements for a visual stimulus under occlusion. This is an exploratory investigation, and as such there were manipulations to pitch, and also stimulus speed and landmark position. I also manipulated whether stimulus movement occurred in a horizontal or vertical plane. The aim was to see how these parameters influence TTC estimates, and identify conditions that show maximal effects of pitch on predicted motion. The parameters showing positive effects could then be used in Experiment 2 in which TMS was used to disrupt integration.

Participants were engaged in a TTC task, in which a moving dot stimulus disappeared behind an occluder (a black rectangle). Participants were asked to make a response at the time they judged the moving dot to have come into contact with a marked point (the landmark). The TTC was taken as the point at which the centre of the 
visual stimulus (its point of origin) would have come into contact with the centre of a landmark, located at one of three positions on the occluding rectangle. The stimulus could move horizontally (rightward, leftward) or vertically (downward, upward). Directionality was investigated in separate, counterbalanced blocks, whereas other variables were manipulated within blocks. On sound trials, a $500 \mathrm{~Hz}$ tone was paired with the visual stimulus, starting at the same time as the onset of movement. When the visual stimulus disappeared behind the occluder, the auditory stimulus could change frequency (rise or fall) or remain constant. Because sound can alter visual judgements (Prime \& Harris, 2010; Teramoto et al., 2010; and Wuerger et al., 2010), it was expected that occlusion would only increase these effects. In particular, during horizontal trials when the visual stimulus is occluded, a rise in pitch should speed up predicted motion (Collier \& Hubbard, 1998). This alteration in predicted speed should be evidenced by a premature TTC response (underestimation), in which participants judge the stimulus to have contacted the landmark point before it actually would have. However, a falling pitch should evoke the sensation of a slower stimulus and therefore a delayed response (overestimation), evidenced by participants pressing the required key after the stimulus would have already contacted the landmark.

However, under vertical movement, different results may be observed. There are two reasons for this. Firstly, pitch is mapped vertically in the visual field, with high pitched tones mapped in the upper visual field and low tones mapped in the lower visual field (Chiou \& Rich, 2012). Therefore, a rising pitch may produce the illusion of upward stimulus motion, and a falling pitch the illusion of downward motion. Thus, although the direction of stimulus movement (leftward or rightward) may not matter in horizontal trials, it may be critical in vertical trials. Secondly, there is another process to consider when using vertical stimulus movement: gravity. For vertical stimuli, there should be underestimations when stimuli are moving downward because of faster speed estimates due to an imagined pull of gravity, as suggested by the internal gravity model (Zago et al., 2010; Zago \& Lacquaniti, 2005a). Furthermore, if this is the case, then upward moving stimuli should react in the opposite fashion; TTC estimates should be slowed as imagined gravity is pulling downward.

If the information we know about pitch and gravity (and their accompanying spatial effects) is combined, then it is possible the estimated TTC will be altered depending on the combination of gravity and pitch effects. 


\section{Method}

\section{Participants}

Participants were 20 (14 female; mean age 25.24 years) adults; all were students or staff at Victoria University of Wellington. All had normal or corrected-to-normal vision and normal hearing. Eighteen were right handed. Approval was granted by the Human Ethics Committee of the School of Psychology, Victoria University of Wellington to carry out this experiment. Participants read an information sheet and gave written consent before experiment participation.

\section{Stimuli and Apparatus}

Experimental stimuli were presented using a Dell PC, on a Samsung $51 \mathrm{~cm} \times 29$ $\mathrm{cm}$ monitor with a refresh rate of $120 \mathrm{~Hz}$. The experiment ran on a 64 bit operating system equipped with Windows 7. The experimental paradigm was constructed using Psychopy (Peirce, 2007) version 1.80.03. Head movements were minimised by using a full-head chin-rest, positioned $57 \mathrm{~cm}$ from the screen.

Dot stimuli were $1 \mathrm{~cm}$ in diameter, pertaining to $1^{\circ}$ of visual angle. These stimuli travelled at speeds of $5.5,8.25$, or $11 \mathrm{~cm} / \mathrm{s}$. These stimulus speeds were based on those of Prime and Harris (2010), who used 5.5 and $11 \mathrm{~cm} / \mathrm{s}$ in their experiment. However, it was preferable to examine time to contact with three different speeds, which could translate into slow, medium and fast movement. Therefore, an additional speed was included in this study.

The visual stimuli travelled within a $27 \mathrm{~cm}$ long track, positioned at the centre of the screen. It was necessary to have the track the same width as the visual stimulus to minimise the effect of pitch on vertical spatial mapping, as high pitched tones have been shown to be mapped onto high spatial positions and low tones mapped low spatially (Chiou \& Rich, 2012). This track ensured participants did not infer an upward or downward stimulus trajectory during horizontal trials (Rusconi et al., 2006). A $17 \mathrm{~cm}$ $\times 1 \mathrm{~cm}$ visual occluder was positioned $5 \mathrm{~cm}$ from the screen's centre. Landmarks could appear at $0.75,5$, or $9.25 \mathrm{~cm}$ from the centre of the screen (at $4.25,8.5$, and $12.75 \mathrm{~cm}$ along the occluder).

Auditory tones were created using Audacity version 2.0.5 (Audacity, 2008). The baseline sound stimulus was $500 \mathrm{~Hz}$, and $4.7 \mathrm{~s}$ in duration. At either $0.86,1.15$ or 1.72 seconds (depending on the speed condition and consistent with the onset of occlusion), the sound could change from $500 \mathrm{~Hz}$ and either steadily increase (rising pitch condition) or decrease in frequency (falling pitch condition) by $21 \mathrm{~Hz} / \mathrm{s}$. In the rising 
pitch condition, the target frequency was $600 \mathrm{~Hz}$, and in the falling, $400 \mathrm{~Hz}$. However, the final frequency was rarely reached as the tone ceased when a response was made. Auditory stimuli were played through Phillips over-ear circumaural headphones.

\section{Procedure}

Participants were seated in a dimly lit room, with their heads secured in a chinrest. A prediction-motion task was used, in which TTC deviation was the dependent variable. On horizontal trials, a dot was presented on either the left or right side of the screen, within a 'track' (two parallel, horizontal lines). A key press initiated the movement, at which time the dot moved from its starting point at one edge of the screen, to the opposite side of the screen until it reached a masking occluder and disappeared from view. A white 'finish line' was located at one of three possible locations on the masking occluder. Participants were required to press the spacebar when they judged the dot to have reached this landmark, assuming that the dot continued moving at the same speed and trajectory behind the mask as it did before occlusion. The stimulus speed was always constant within a single trial and the visual stimulus always moved in a straight line of motion (see Figure 3 for an example).

Participants were instructed to ignore sound when it was present, and base their judgements on the visual stimulus. Furthermore, on each trial the visual stimulus could move at one of three different speeds to one of three landmark positions. This created nine actual TTC times, as shown in Table 1.

The vertical trials were identical to the horizontal trials, except the direction of motion was within the vertical plane. The stimulus could start at the top or bottom of the screen, meaning the stimulus direction could be upward or downward. Prior to the experiment, participants were presented with 36 silent practice trials, 18 with horizontal and 18 with vertical motion. No feedback was given, to prevent participants from calibrating their judgements.

Each block consisted of 360 trials; with 720 trials presented over the duration of the experiment $(2$ stimulus planes [horizontal, vertical] $\times 2$ starting points [left, right/ up, down] $\times 3$ landmark positions [near, middle, far] $\times 3$ stimulus speeds [slow, medium, fast] $\times 4$ pitch conditions [constant, rising, falling, silent]). There were five repetitions of each condition. Block order was counterbalanced between participants, and the other conditions were fully randomised within each block. 

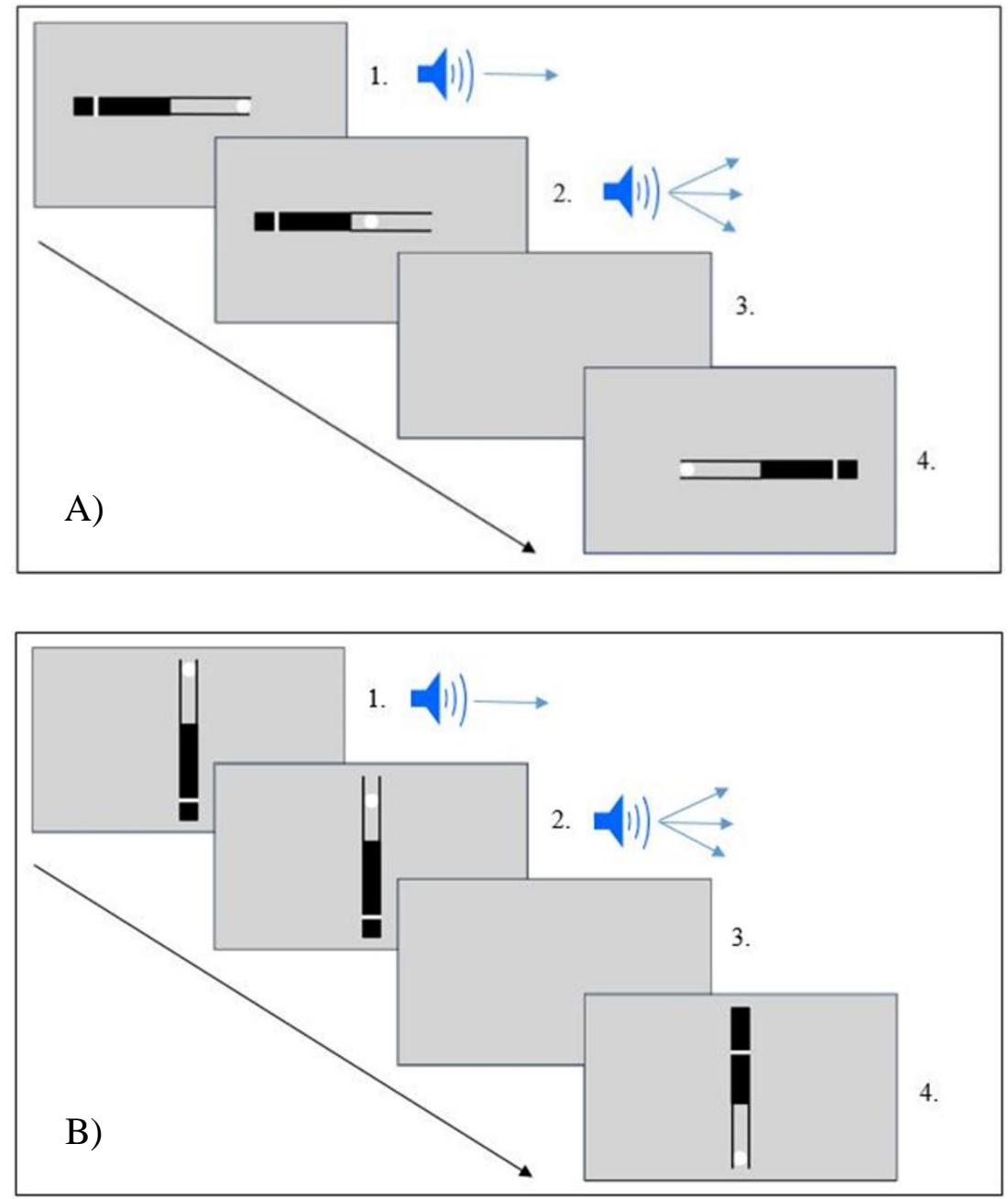

Figure 3. Example of A) a typical horizontal trial; and B) a typical vertical trial (not to scale). Upon key press, the visual stimulus (the white disc) moved along the track towards the black rectangle and became occluded from view. Participants had to make another key press when they estimated the visual stimulus to have reached the white line.

Sequence of events:

1. Spacebar initiates stimulus movement; at the same time a $500 \mathrm{~Hz}$ tone is played. 2. Visual stimulus moves along a constant trajectory, until key press. Pitch stays the same or rises/falls in pitch by $21 \mathrm{~Hz} / \mathrm{s}$.

3. ISI for $0.5 \mathrm{~s}$.

4. New randomised trial appears. 
Table 1

Actual TTC of visual stimulus (in seconds) as a function of landmark position and stimulus speed for Experiments $1 A$ and $1 B$.

Landmark Position

\begin{tabular}{lccc}
\cline { 2 - 4 } Stimulus Speed & Near & Middle & Far \\
\hline Slow & 2.50 & 3.27 & 4.04 \\
Medium & 1.66 & 2.18 & 2.69 \\
Fast & 1.25 & 1.63 & 2.02 \\
\hline
\end{tabular}

\section{Results and Discussion}

The actual time to contact (TTCa) was calculated as the time-point at which the centre of the visual stimulus would have coincided with the centre of the landmark, had it continued to move at a constant speed while occluded (see Table 1). Mean observed TTC values (TTCo) were recorded for each participant for all conditions. These means were used to calculate TTC error values (TTCe), in which the actual TTC (TTCa) was taken away from the observed TTC (TTCo). By this method, positive values indicate overestimations in seconds (i.e. the visual stimulus is estimated to be moving slower than it actually is [TTCo > TTCa]), and negative values represent underestimations (i.e. the visual stimulus is estimated to be moving faster than it actually is [TTCo $<$ TTCa]). Perfect accuracy would produce no difference between the actual TTC and the estimated TTC (the error would be zero). Using TTC error values gives a more indicative view of performance compared to actual TTC values in seconds, as it enables us to see how far responses deviate from the actual TTC (Hofbauer et al., 2004).

Trials in which responses were made before the occlusion of the visual stimulus were deemed anticipatory and removed from the analysis. Furthermore, trials in which participants made a response 1.5 seconds before or after the actual TTC were deemed to be indicative of distraction from the task and were removed from the analysis ${ }^{1}$. Participants for whom $10 \%$ or more of trials were deleted were excluded from the

\footnotetext{
${ }^{1}$ Gordon \& Rosenblum (2005) removed trials if TTC was inaccurate by more than 2.5 seconds. A stricter approach was adopted here.
} 
analysis. Additionally, participants for whom four or more trials were deleted in a single condition were also removed from the analysis. No participants were excluded based on these criteria in Experiment 1A. Analyses are therefore based on all 20 participants. The average rate of rejection was $0.64 \%$ of trials per participant in the horizontal condition $(S D=1.29 \%$, range $=0-5.56 \%)$ and $1.56 \%$ in the vertical condition $(S D=2.34 \%$, range $=0-7.78 \%)$.

Mean TTCe for each condition are presented in Appendices A and B. Although this experiment featured a within-subject design, the horizontal and vertical trials were analysed separately, as it was expected that pitch could have qualitatively different effects on horizontal and vertical stimulus movement. Degrees of freedom were adjusted using the Greenhouse-Geisser correction when violations of sphericity occurred. All post-hoc t-tests were paired-samples, with an adjusted alpha level of $\alpha=$ .016 , unless otherwise specified.

\section{Horizontal Trials}

TTC Performance: All Pitch Conditions. Performance in the TTC task in pitch trials (rising, falling, or constant) compared to silent trials was of primary interest in this study. Specifically, the aims of the analysis were to determine: (1) whether pitch changes altered the speed of predicted motion over and above that induced by a constant pitch, and (2) whether the effect of pitch differed depending on object speed or landmark location. Analysis therefore focused on main effects and interactions involving the pitch conditions.

TTC performance was first compared across all pitch conditions (constant, rising, falling, silent), to determine the general pattern of deviations from true TTC in terms of direction (underestimations/overestimations) and magnitude of error. As this was not the main analysis, interactions involving pitch will be described in detail in a secondary ANOVA which used silent trials as a baseline measure of performance. This ANOVA will be used to focus on other factor interactions.

A 2 (stimulus direction: left, right) $\times 3$ (landmark position: near, middle, far) $\times 3$ (stimulus speed: slow, medium, fast) $\times 4$ (pitch condition: constant, rising, falling, silent) repeated measures analysis of variance (ANOVA) was conducted to determine TTC deviation (in seconds) for all pitch conditions (including the silent condition).

All conditions produced overestimations relative to the actual TTC (See table A1 in Appendix A). There were main effects of pitch, $F(3,57)=8.31, p<.001, \eta p^{2}$ $=.30$, and speed, $F(1.12,21.22)=22.19, p<.001, \eta p^{2}=.54$, subsumed by a Pitch $\times$ 
Speed interaction, $F(3.70,70.30)=5.67, p=.001, \eta p^{2}=.23$, as shown in Figure 4. This interaction will be explored in the secondary ANOVA.

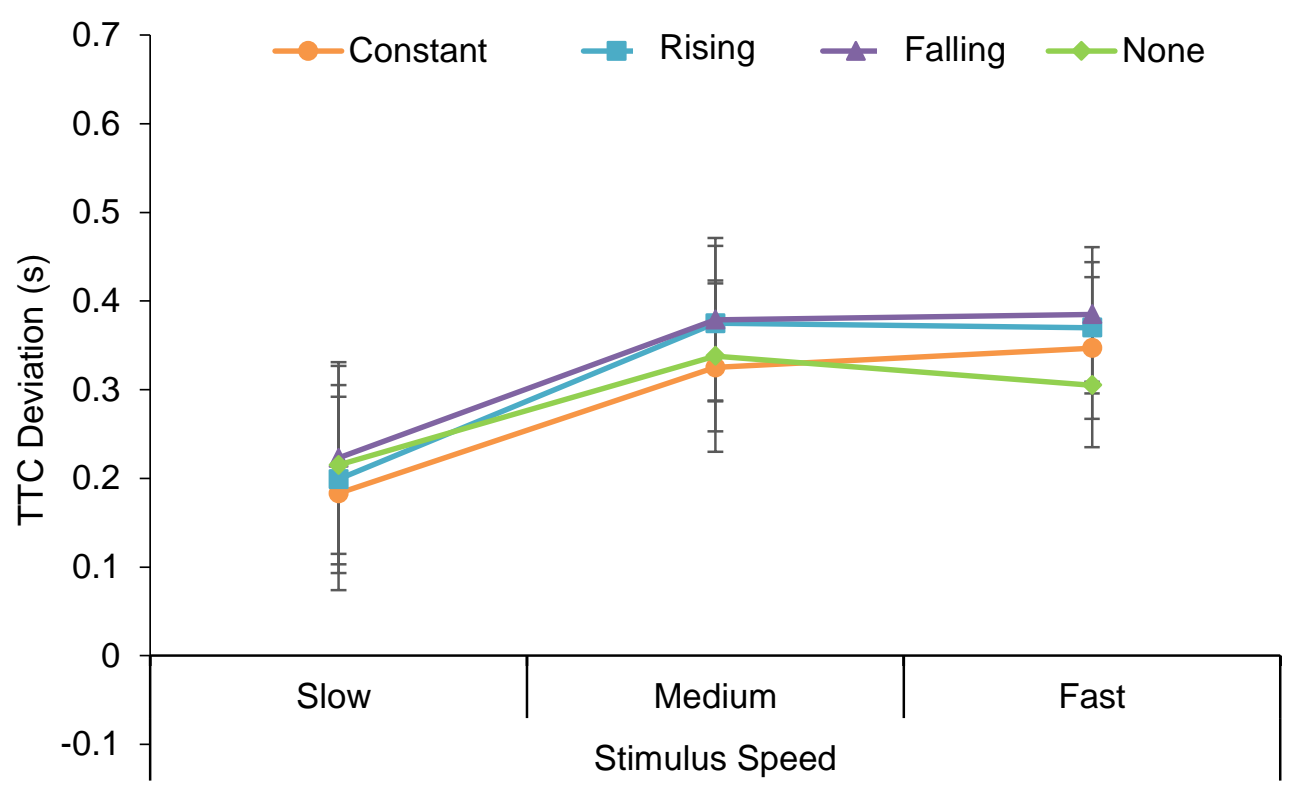

Figure 4. Deviations in TTC estimates for visual stimuli for constant, rising, falling and silent conditions as a function of stimulus speed for horizontal trials in Experiment 1A. The error bars represent the standard error.

There was no main effect of landmark, $F(1.17,22.20)=.41, p=.559, \eta p^{2}=.02$. However, there was a Landmark $\times$ Speed interaction, $F(1.98,37.60)=41.14, p<.001$, $\eta p^{2}=.68$, shown in Figure 5. One-way repeated measures ANOVAs split by landmark showed no effect of speed at the near landmark, $F(1.30,24.72)=1.04, p=.339, \eta p^{2}$ $=.05$, however there was at the middle, $F(1.15,21.88)=16.18, p<.001, \eta p^{2}=.46$, and far landmark positions, $F(1.19,22.57)=38.79, p<.001, \eta p^{2}=.67$. As shown in Figure 5 , at the middle landmark, a slow speed produced signifi cantly smaller overestimations compared to fast, $t(19)=-4.02, p=.001, d=-0.90$, and medium speeds, $t(19)=-4.59, p$ $<.001, d=-1.03$, which did not differ from each other, $t(19)=1.99, p=.062,0.44$. At the far landmark, slow speed also produced smaller overestimations compared to fast, $t(19)=-6.22, p<.001, d=-1.39$, and medium speeds, $t(19)=-7.33, p<.001, d=-1.64$. Medium speed also produced smaller overestimations than fast speed, $t(19)=2.64, p$ $=.016, d=0.59$. 
There was also a Landmark $\times$ Pitch interaction, $F(6,114)=2.98, p=.010, \eta p^{2}$ $=.14$, shown in Figure 6. This interaction will also be explored in the secondary ANOVA. All other interactions were nonsignificant.

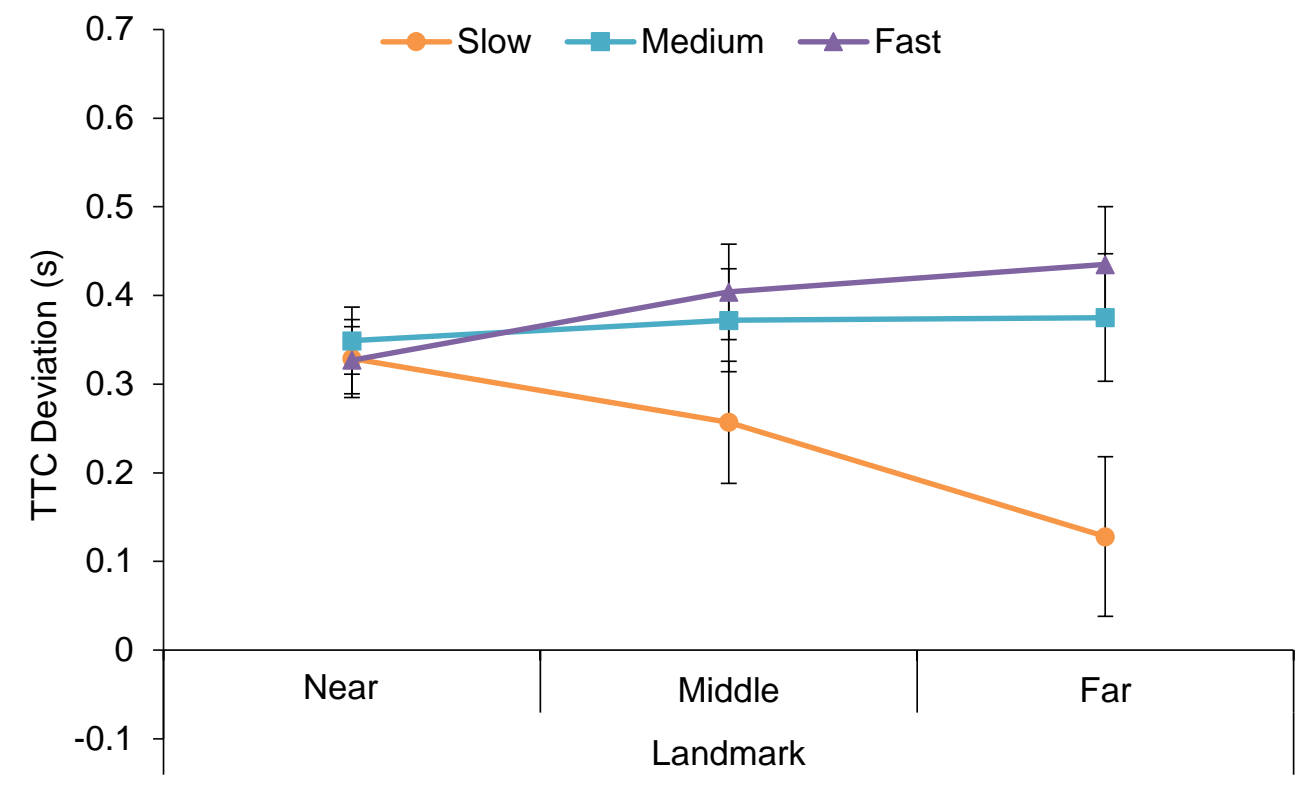

Figure 5. Deviations in TTC estimates for visual stimuli for slow, medium and fast speeds as a function of landmark position for horizontal trials in Experiment 1A. The error bars represent the standard error.

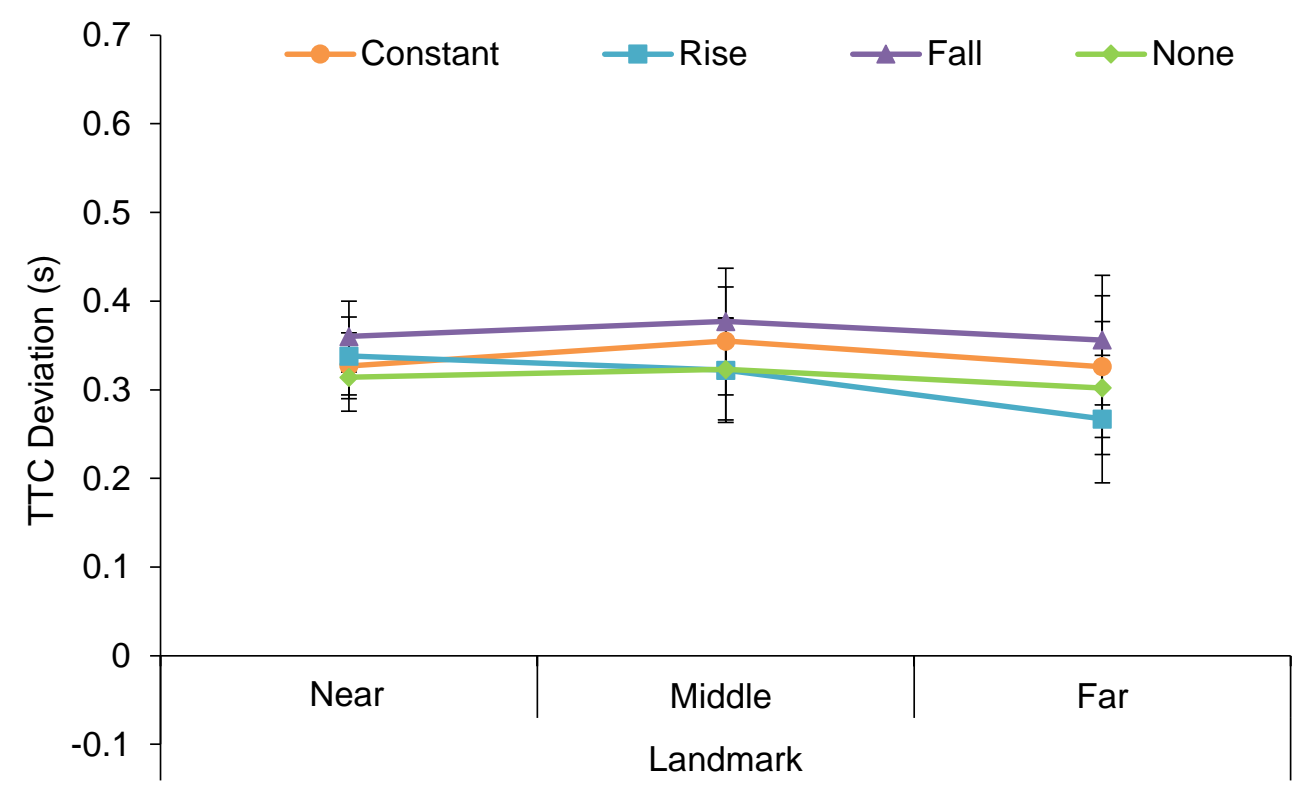

Figure 6. Deviations in TTC estimates for visual stimuli for constant, rising, falling and silent conditions as a function of landmark position for horizontal trials in Experiment 1A. The error bars represent the standard error.

TTC Performance: Relative to Silent Baseline. The first analysis revealed consistent overestimations in TTC values. However, the research question focuses on 
the effects of pitch on predicted motion. Therefore, to investigate how pitch alters TTC motion imagery relative to silent trials, the overall overestimation bias was removed by subtracting the TTC deviation in the silent trials from deviation values for the remaining three pitch conditions (constant, rising, and falling). This method gave TTC deviations relative to the silent condition. Positive values now indicate that predicted motion was overestimated relative to the silent condition (that is, pitch produced slower predicted motion than no pitch) and negative values indicate that predicted motion was underestimated relative to baseline (that is, pitch produced faster predicted motion than no pitch). Analysis here focused on two questions. First, did TTC differ when accompanied by a rising and a falling pitch, and second, did a rising or a falling pitch affect TTC relative to a constant pitch.

A 2 (stimulus direction: leftward, rightward) $\times 3$ (landmark position: near, middle, far) $\times 3$ (stimulus speed: slow, medium, fast) $\times 3$ (pitch condition: constant, rising, falling) repeated measures ANOVA was used to compare relative deviations in TTC. Importantly, there was no main effect of direction in the horizontal condition, $F(1,19)=0.26, p=.259, \eta p^{2}=.07$.

There was a main effect of pitch, $F(2,38)=9.34, p=.001, \eta p^{2}=.33$, and a main effect of speed, $F(2,38)=10.08, p<.001, \eta p^{2}=.35$, subsumed by a Pitch $\times$ Speed interaction, $F(4,76)=3.93, p=.006, \eta p^{2}=.17$. This interaction was driven by underestimations in TTC for rising pitch at the slow speed, and overestimations for falling pitch at the fast speed. To explore this interaction, one-way repeated measures ANOVAs were conducted for the slow, $F(2,38)=5.65, p=.007, \eta p^{2}=.23$, and fast stimulus speeds, $F(2,38)=9.68, p<.001, \eta p^{2}=.34$. As shown in Figure 7, although a rising pitch produced underestimations relative to a falling pitch at all speeds, they differed with respect to their effects relative to the constant pitch. At slow speeds, a rising pitch produced underestimations compared to a falling, $t(19)=-2.73, p=.013, d$ $=-0.61$, and a constant pitch, $t(19)=-3.57, p=.002, d=-0.80$, which did not differ, $t(19)=-.24, p=.810, d=-0.05$, suggesting the pitch effect is driven by the rising pitch which produced speeded predicted motion relative to a constant pitch. However, at the fast speed, a falling pitch produced overestimations compared to a rising, $t(19)=2.83, p$ $=.011, d=0.63$, and a constant pitch, $t(19)=4.84, p<.001, d=1.08$, which did not differ, $t(19)=1.07, p=.297, d=0.24$, suggesting the pitch effects were driven by the falling pitch at this speed, indicating slowed predicted motion relative to a constant pitch. 


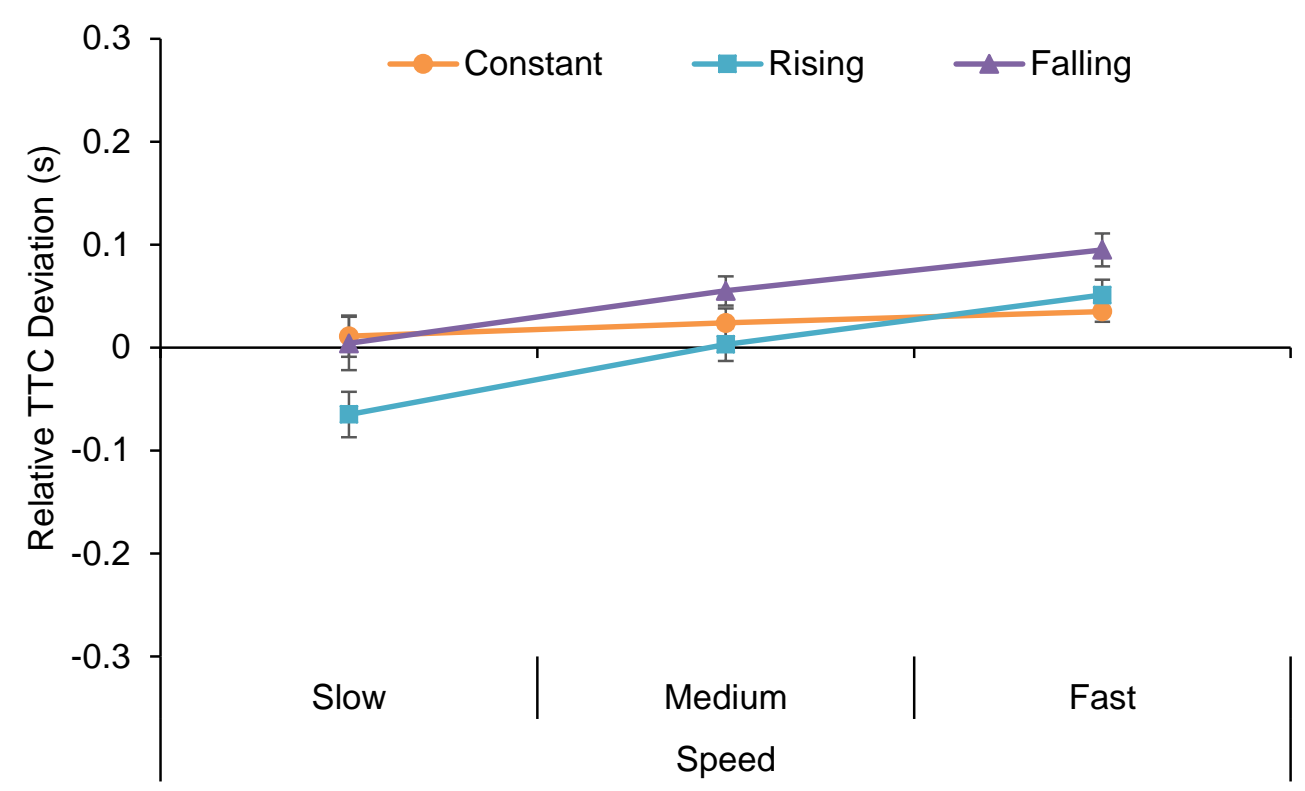

Figure 7. Relative deviations in TTC estimates for visual stimuli for constant, rising, and falling conditions as a function of stimulus speed for horizontal trials in Experiment 1A. The error bars represent the standard error.

Inherent pitch-speed associations tell us objects that emit a rising pitch are expected to be moving faster than objects emitting a falling pitch (Collier \& Hubbard, 1998). That effect is evident here, as a rising pitch always produced underestimations relative to a falling pitch. However, the speed of the object influenced whether the pitch produced an overestimation or underestimation relative to a constant pitch. It could be that a changing pitch is ignored if it does not add any extra information. That is, at the slow stimulus speed, a falling pitch is the expected accompaniment and is not processed further than to confirm that the object is moving slowly. However, a rising pitch is incongruent with the original speed, causing a bias in the predicted motion of the object. The same explanation could be used at the fast stimulus speed. In this situation, the rising pitch matches the stimulus speed; both indicate the object is fast-moving. Therefore, it is the falling pitch that causes a speed bias, because it provides additional information that does not adhere to expectations. There is evidence to suggest one sensory modality is given preference over the other when performance in one modality is degraded or becomes unavailable (Heron, Whitaker, \& McGraw, 2004). This evidence provides support for pitch biasing the stored speed information, suggesting that when visual information stops being available, pitch information can change the remembered speed of the visual stimulus. 
Pitch also interacted with landmark position, $F(4,76)=4.62, p=.002, \eta p^{2}=.20$, indicating consistent TTC estimations across landmark positions for a constant and falling-pitch, but decreasing TTC estimations across landmarks for a rising pitch, resulting in underestimations at the far landmark, as shown in Figure 8. One-way repeated-measures ANOVAs split by landmark indicated the main effect of pitch to be significant at each of the near, $F(2,38)=3.51, p=.040, \eta p^{2}=.16$, middle, $F(2,38)=$ $6.50, p=.004, \eta p^{2}=.26$, and far landmark positions, $F(2,38)=9.14, p=.001, \eta p^{2}=.33$.

However, pitch effects were greatest at the far landmark. A falling pitch produced overestimations comparing to a rising pitch at the middle, $t(19)=3.36, p$

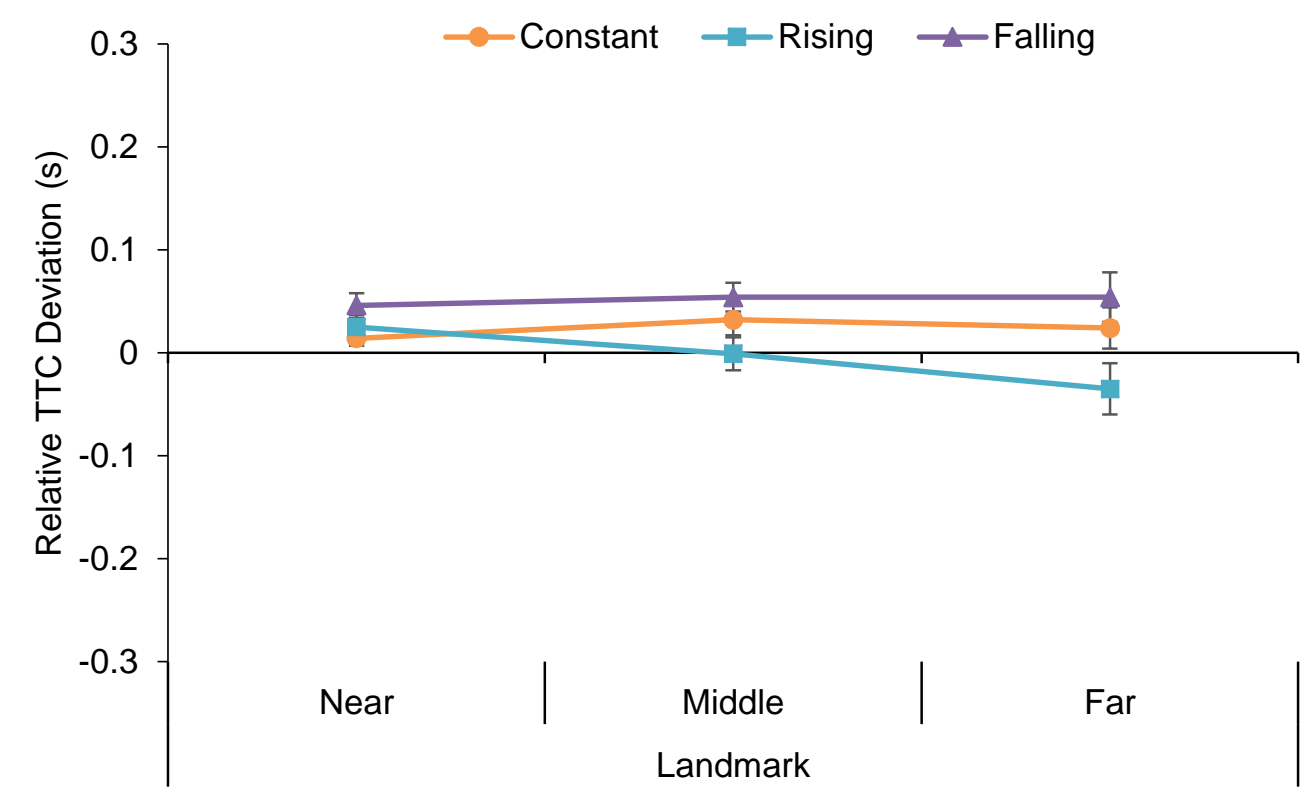

Figure 8. Relative deviations in TTC estimates for visual stimuli for constant, rising, and falling conditions as a function of landmark for horizontal trials in Experiment 1A. The error bars represent the standard error.

$=.003, d=0.75$, and far landmarks, $t(19)=3.88, p=.001, d=0.87$, showing the predicted pitch effect, but not at the near landmark, $t(19)=1.99, p=.062, d=0.44$.

However at the near landmark, a falling pitch produced overestimations compared to a constant pitch, $t(19)=2.66, p=.015, d=0.60$, whereas a rising pitch did not, $t(19)=$ -.76, $p=.455, d=0.17$. At the middle landmark, neither a falling, $t(19)=1.53, p$ $=.144, d=0.34$, nor a rising pitch differed from a constant pitch, $t(19)=-2.15, p$ $=.044, d=-0.48$. At the far landmark, a falling pitch did not produce significant overestimations compared to a constant pitch, $t(19)=1.38, p=.183, d=0.31$, but a rising pitch did, $t(19)=3.20, p=.005, d=0.71$. This result indicates that the pitch 
effect is primarily driven by the rising pitch, producing speeded predicted motion relative to a constant pitch.

To summarise, the effect of pitch on predicted motion was largely as predicted. A rising pitch produced speeded predicted motion relative to a falling pitch across most conditions, although the direction of the effects relative to a constant pitch differed as a function of speed and landmark position. Overall, pitch and speed effects were greatest at the farthest landmark. The interaction between pitch and speed showed that a rising pitch speeded predicted motion at the slow stimulus speed and a falling pitch slowed predicted motion at the fast stimulus speed. Findings in the horizontal condition are therefore consistent with the hypothesis that predicted motion integrates auditory and visual components, producing distortions in imagined speed.

\section{Vertical Trials}

TTC Performance: All Pitch Conditions. As with the horizontal trials, TTC performance was first compared across all pitch conditions (constant, rising, falling, silent), to determine the general pattern of deviations. Again, overlapping interactions involving pitch are described in detail in the secondary ANOVA which used silent trials as a baseline measure of performance.

A 2 (stimulus direction: upward, downward) $\times 3$ (landmark position: near, middle, far) $\times 3$ (stimulus speed: slow, medium, fast) $\times 4$ (pitch condition: constant, rising, falling, silent) repeated measures ANOVA was also used to compare TTC deviations (in seconds) for TTC estimates.

All conditions produced overestimations (see Table A2 of the appendix for mean TTC deviations for each condition). There was a main effect of direction, $F(1,19)$ $=24.20, p<.001, \eta p^{2}=.56$, in which downward moving stimuli $(M=0.31, S D=0.29)$ were judged as moving faster than upward moving stimuli $(M=0.39, S D=0.29)$. This effect is supported by the view of an internal gravity model (Zago et al., 2010, McIntyre et al., 2001). There was a Direction $\times$ Landmark interaction, $F(1.46,27.80)=9.51, p$ $=.002, \eta p^{2}=.33$. As indicated in Figure 9, at the near landmark, upward motion produced greater overestimations in TTC than downward motion, $t(19)=2.47, p=$ $.023, d=0.55$. The same pattern was observed at the middle, $t(19)=4.78, p<.001, d=$ 
1.07, and far landmarks, $t(19)=4.38, p<.001, d=0.98$, however the effect size was much larger at these landmarks $(\alpha=0.05)$.

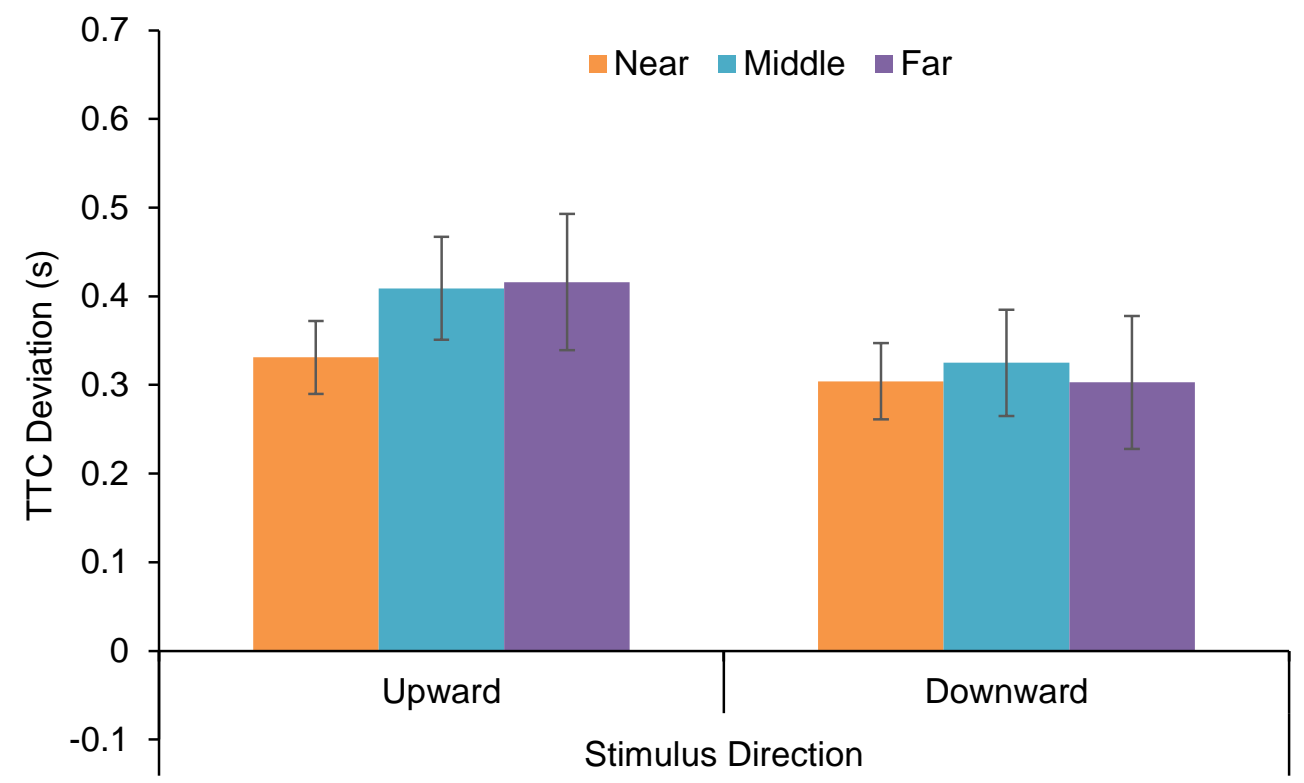

Figure 9. Deviations in TTC for visual stimuli at near, middle and far landmark positions for upward and downward stimulus motion in Experiment 1A for vertical trials. Error bars represent the standard error.

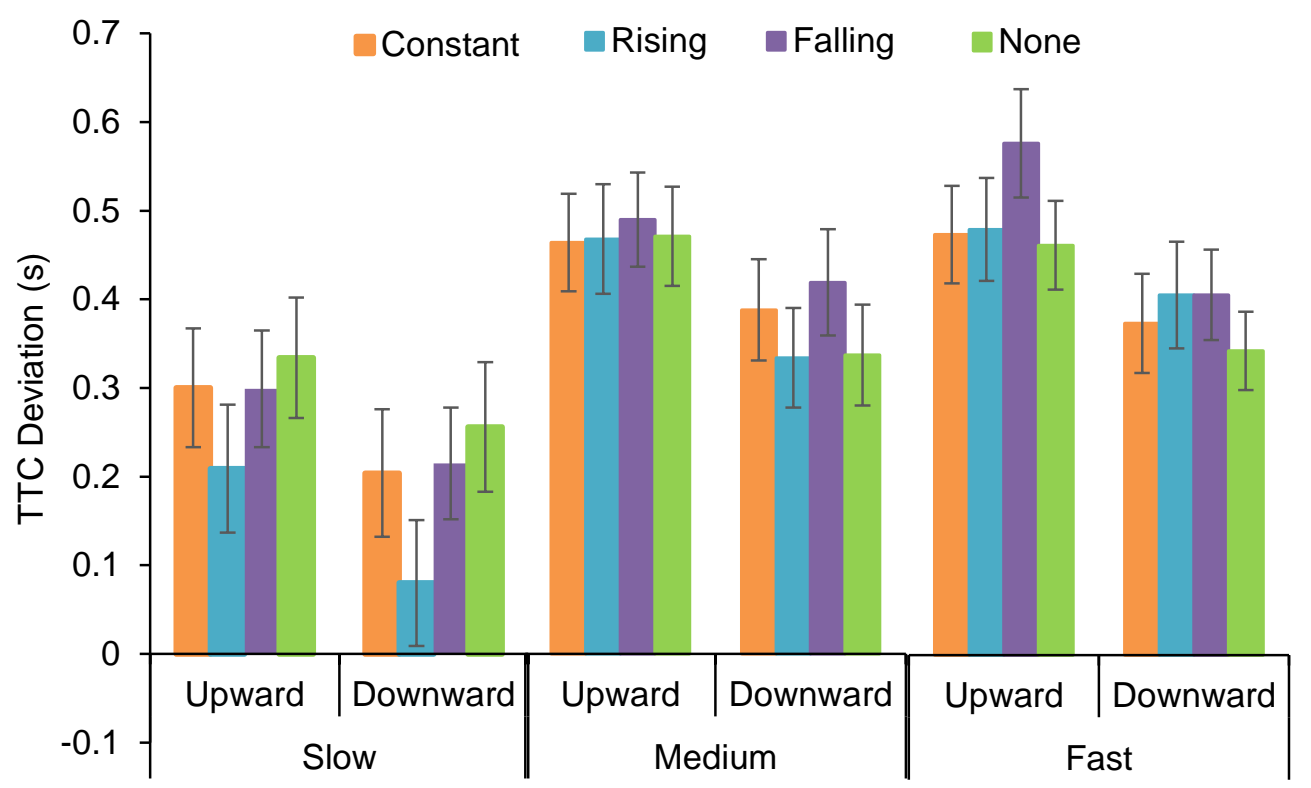

Figure 10. Relative deviations in TTC estimates for visual stimuli in the constant, rising, falling and silent conditions at slow, medium and fast stimulus speeds, for upward and downward motion in Experiment 1A for vertical trials. The error bars represent the standard error.

There were main effects of pitch, $F(3,57)=8.59, p<.001, \eta p^{2}=.31$, and speed, $F(1.14,21.73)=30.00, p<.001, \eta p^{2}=.61$, contained within a Pitch $\times$ Speed interaction, $F(3.46,65.81)=13.58, p<.001, \eta p^{2}=.42$, and a Direction $\times$ Pitch $\times$ Speed 
interaction, $F(3.08,58.42)=3.29, p=.026, \eta p^{2}=.15$, shown in Figure 10. These interactions will be examined in the secondary ANOVA.

There was no main effect of landmark position, $F(1.13,21.41)=1.14, p=.307$, $\eta p^{2}=.06$. There were however, Landmark $\times$ Pitch, $F(6,114)=3.68, p=.002, \eta p^{2}=.16$, and Landmark $\times$ Speed interactions, $F(2.11,39.99)=39.76, p<.001, \eta p^{2}=.68$. These were contained within a Landmark $\times$ Pitch $\times$ Speed interaction, $F(6.19,117.65)=4.37$, $p<.001, \eta p^{2}=.19$, shown in Figure 11. This interaction will also be explored in depth in the secondary ANOVA.

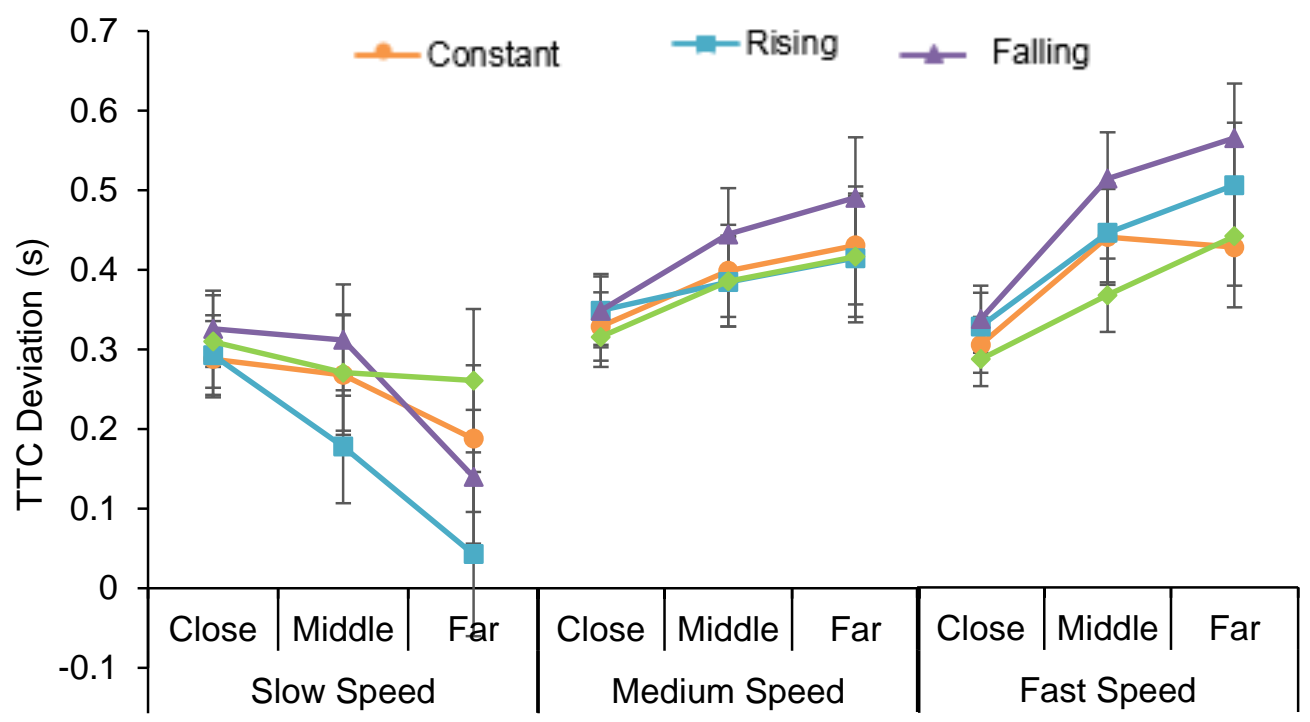

Figure 11. Deviations in TTC for visual stimuli in constant, rising, falling and silent trials by landmark position at slow, medium and fast stimulus speeds in Experiment $1 \mathrm{~A}$ for vertical trials. Error bars represent the standard error.

TTC Performance: Relative to Silent Baseline. The first analysis showed mean TTC deviations were positive for vertical motion (as with horizontal) indicating overestimations overall. Data was transformed to produce TTC deviations relative to the silent condition in the same manner as for horizontal trials. Positive values therefore reflect overestimations (relative to silent baseline), and negative values reflect underestimations. Again, analyses focused on two questions; did TTC differ as a function of risi Near'sus falling pitch, Near ow did pitch effe Near mpare to a constant pitch.

A 2 (stimulus direction: upward, downward) $\times 3$ (landmark position: near, middle, far) $\times 3$ (stimulus speed: slow, medium, fast) $\times 3$ (pitch condition: constant, rising, falling) repeated measures ANOVA was used to compare deviations in TTC relative to silent trials. There were main effects of pitch, $F(2,38)=11.85, p<.001, \eta p^{2}$ 
$=.38$, and speed, $F(2,38)=22.77, p<.001, \eta p^{2}=.55$, that were qualified by a Pitch $\times$ Speed interaction, $F(2.52,47.85)=8.16, p<.001, \eta p^{2}=.30$.

There was no main effect of direction in this analysis, $F(1,19)=0.02, p=.90$, $\eta p^{2}=.001$, due to subtracting the silent trials. However, there was a significant Direction $\times$ Pitch interaction, $F(2,38)=3.26, p=.049, \eta p^{2}=.15$, indicating that the stimulus direction affected the magnitude of the pitch effect. The Direction $\times$ Pitch, and Pitch $\times$ Speed interactions described were subsumed within a Direction $\times$ Pitch $\times$ Speed interaction, $F(2.27,43.17)=3.94, p=.026, \eta p^{2}=.17$, which was driven by underestimations for a rising pitch at the slow speed and overestimations for a falling pitch at the slow speed. To further explore the three-way interaction, Direction $\times$ Pitch ANOVAs were conducted at the slow and fast condition separately. There was a trending Direction $\times$ Pitch interaction at the slow speed, $F(2,38)=2.96, \mathrm{p}=.064, \eta p^{2}$ $=.14$, and a significant Direction $\times$ Pitch interaction at the fast speed, $F(2,38)=4.42, p$ $=.019, \eta p^{2}=.19$.

Following up the two Direction $\times$ Pitch $\times$ Speed interactions, at the slow stimulus speed, a rising pitch produced underestimations compared to a falling pitch, $t(19)=-5.06, p<.001, d=-1.13$, and a constant pitch, $\mathrm{t}(19)=-4.05, p=.001, d=-0.91$, which did not differ from each other, $t(19)=.60, p=.558, d=0.13$, as seen in Figure 12.

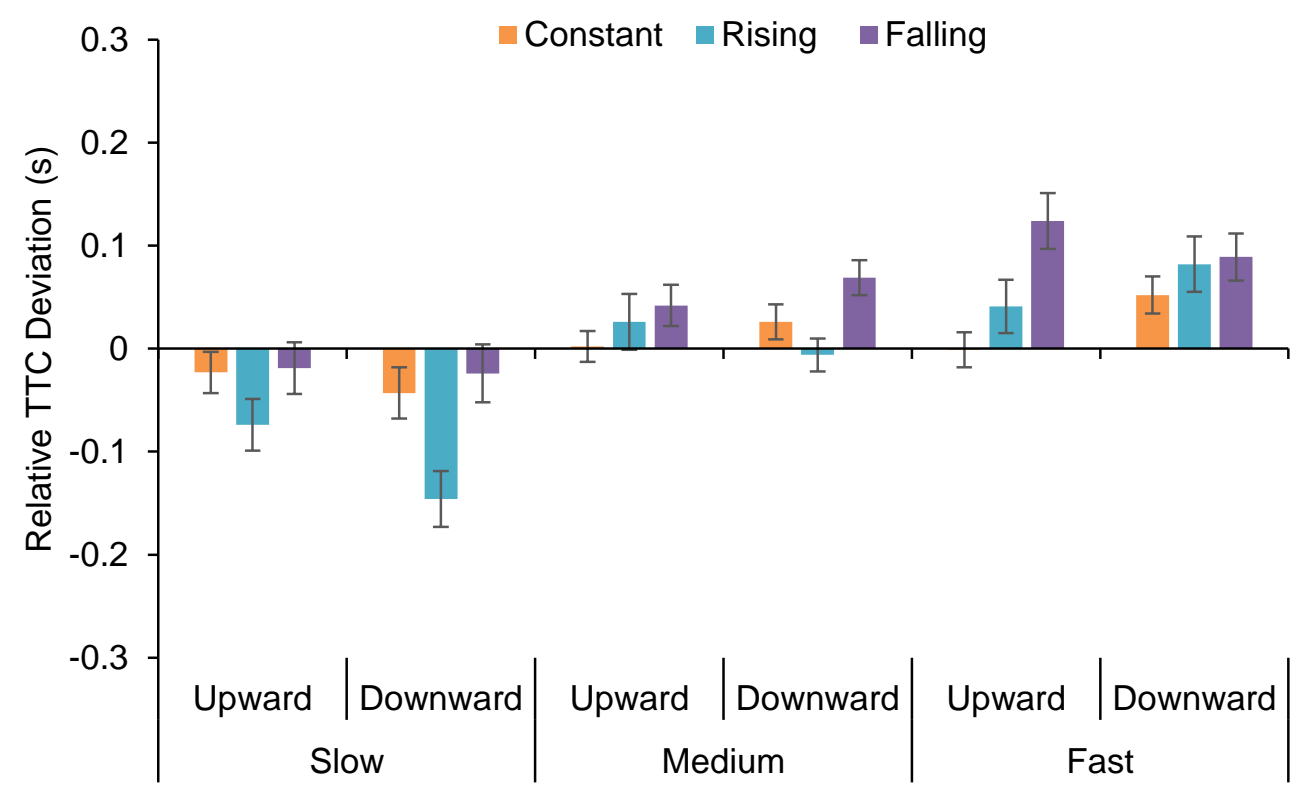

Figure 12. Relative deviations in TTC for visual stimuli in constant, rising, and falling trials by stimulus speed for upward and downward movement in Experiment 1A for vertical trials. Error bars represent the standard error. 
This suggests a pitch effect which is driven by the rising pitch, producing speeded predicted motion. At the fast stimulus speed, a falling pitch produced overestimations compared to a rising pitch, $t(19)=2.97, p=.008, d=0.66$, and a constant pitch, $t(19)=4.64, p<.001, d=1.04$, which did not differ from each other, $t(19)=-1.80, p=.088, d=-0.40$. This suggests the pitch effect is driven by the falling pitch, which produced slowed predicted motion. These effects are similar to those seen in the horizontal condition, in that a rising pitch primarily affected predicted motion of slow-moving objects, and a falling pitch primarily affected predicted motion of fastmoving objects. These pitch effects were further qualified by direction. At the slow stimulus speed, a rising pitch produced larger underestimations for downward than upward movement, and at the fast stimulus speed, a falling pitch produced larger overestimations for upward compared to downward moving stimuli, consistent with a gravity effect. However, in paired-samples t-tests $(\alpha=0.05)$, the direction effect did not reach statistical significance for TTC estimates for either rising, $t(19)=2.01, p=.059$, $d=0.45$, or a falling pitch, $t(19)=1.07, p=.297, d=0.24$.

There was also a main effect of landmark, $F(2,38)=6.06, p=.005, \eta p^{2}=.24$, a Landmark $\times$ Pitch interaction, $F(4,76)=2.95, p=.026, \eta p^{2}=.13$, and a Landmark $\times$ Speed interaction, $F(2.73,51.79)=4.56, p=.008, \eta p^{2}=.19$. These two-way interactions were subsumed within a Landmark $\times$ Pitch $\times$ Speed interaction, $F(8,152)=$ 4.22, $p<.001, \eta p^{2}=.18$. This interaction was driven by underestimations for a rising pitch at the far landmark for slow speed stimuli, and overestimations for a falling pitch at the far landmark for fast speed stimuli. To further explore this interaction, three Landmark $\times$ Pitch ANOVAs were conducted split by speed, finding signficant interactions between the two at the slow, $F(4,76)=5.19, p=.001, \eta p^{2}=.21$, and fast speeds, $F(4,76)=3.05, p=.022, \eta p^{2}=.14$, but not at the middle speed, $F(4,76)=1.81$, $p=.135, \eta p^{2}=.09$. 
Figure 13 shows at the slow stimulus speed, a falling pitch produced overestimations compared to a rising pitch at the middle, $t(19)=4.98, p<.001, d=$ 1.11 , and far landmarks, $t(19)=2.84, p=.011, d=0.63$, showing the general pitch effect. However there was no significant difference between a falling and a rising pitch at the near landmark, $t(19)=2.00, p=.060, d=0.45$. However, at the near landmark, a falling pitch produced overestimations compared to a constant pitch, $t(19)=2.71, p$ $=.014, d=0.61$, suggesting slowed predicted motion. There was no difference between TTC for a constant and a rising pitch at this landmark, $t(19)=-.26, p=.800, d=-0.06$. At the middle landmark and far landmarks, a rising pitch produced underestimations compared to a constant pitch $(t(19)=-3.19, p=.005, d=-0.71$, and $t(19)=-3.84, p$ $=.001, d=-0.86$ respectively), but there was no difference between TTC for a constant and a falling pitch $(t(19)=1.32, p=.203, d=0.30$, and $t(19)=-1.35, p=.194, d=-$ 0.30 respectively), indicating the pitch effects are driven by the rising pitch, which produced speeded predicted motion.

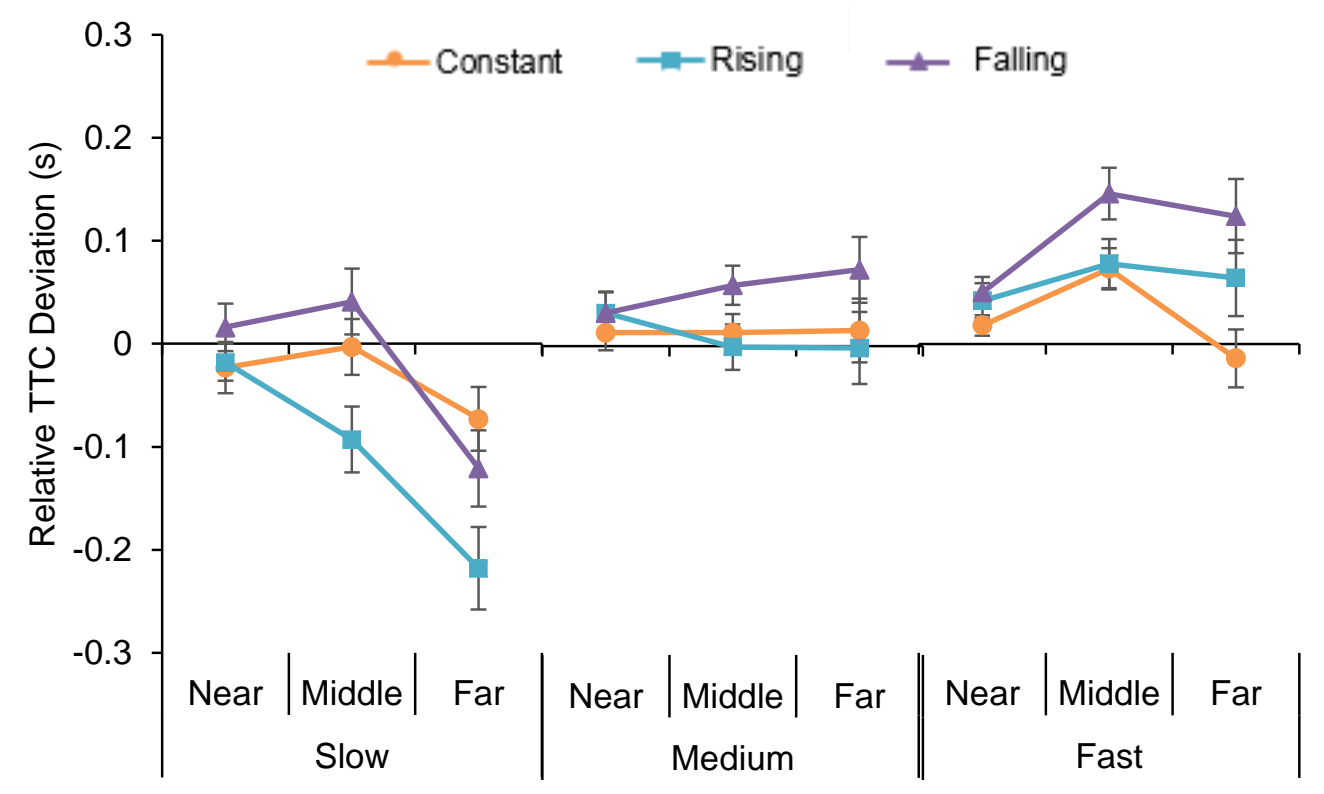

Figure 13. Relative deviation in TTC estimates for visual stimuli in the constant, rising and falling pitch conditions by landmark position at slow, medium and fast speeds in Experiment 1A for vertical trials. Error bars represent the standard error.

At the fast stimulus speed, there were no significant differences between a rising and falling pitch at the near, $t(19)=-.82, p=.425, d=-0.18$, middle, $t(19)=-$ $2.29, p=.034, d=-0.51$, or far landmarks, $t(19)=-1.90, p=.073, d=-0.42$, showing no general pitch effects. However, a falling pitch produced overestimations compared to a constant pitch at the middle, $t(19)=3.27, p=.004, d=0.72$, and far landmarks, 
$t(19)=4.04, p=.001, d=0.90$, but a rising pitch did not produce underestimations compared to a constant pitch, $(t(19)=2.16, p=.044, d=0.48$, and $t(19)=.20, p=.845$, $d=0.04$ respectively), suggesting a speed effect in which falling pitch slowed predicted motion. There was no speed effect at the near landmark position as predicted motion was not affected by falling, $t(19)=1.85, p=.080, d=0.41$, or a rising pitch compared to a constant pitch, $t(19)=1.45, p=.163, d=0.32$.

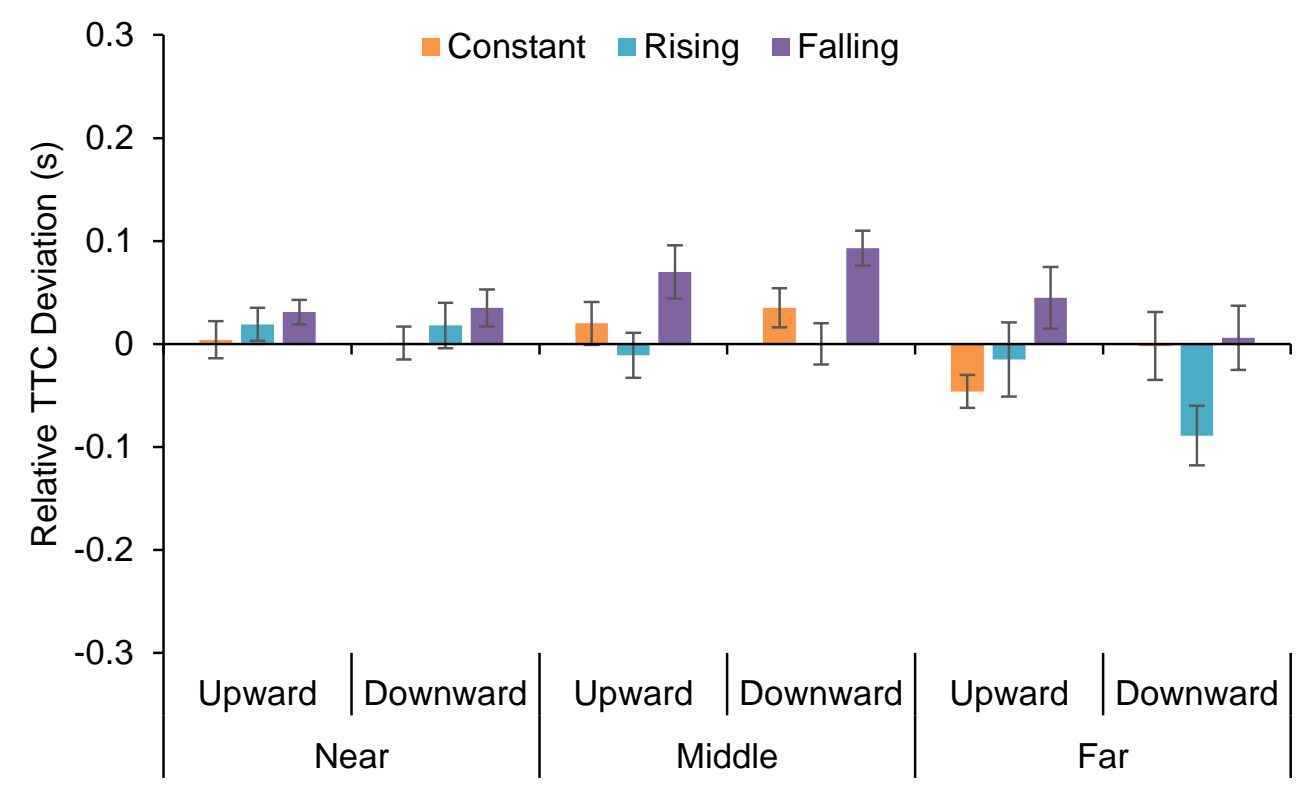

Figure 14. Relative deviation in TTC estimates for visual stimuli in the constant, rising and falling pitch conditions by landmark position for upward and downward motion in Experiment 1A for vertical trials. Error bars represent the standard error.

The ANOVA also revealed a three way interaction between stimulus direction, landmark position and pitch condition, $F(4,76)=3.88, p=.006, \eta p^{2}=.17$, driven by the far landmark position. Three Direction $\times$ Pitch ANOVAs were conducted, showing no interaction at the near, $F(2,38)=.03, p=.970, \eta p^{2}=.00$, or middle landmarks, $F(2$, $38)=.11, p=.894, \eta p^{2}=.01$, but there was at the far landmark, $F(2,38)=9.88, p$ $<.001, \eta p^{2}=.34$.

As shown in Figure 14, at the near and middle landmarks there were generally overestimations regardless of direction, which were largest for a falling pitch. At the far landmark, a rising pitch produced underestimations compared to a falling pitch for downward motion, $t(19)=-3.17, p=.005, d=-0.71$, indicating a pitch effect. This difference was not observed for upward motion, $t(19)=-2.39, p=.027, d=-0.53$. However, for upward motion, a falling pitch produced overestimations compared to a constant pitch, $t(19)=3.59, p=.002, d=0.80$, which did not differ from a rising pitch, 
$t(19)=-1.00, p=.329, d=-0.22$, indicating slowed predicted motion. The converse was observed for downward motion; a rising pitch produced underestimations compared to a constant pitch, $t(19)=-3.40, p=.003, d=-0.76$, which did not differ from a falling pitch, $t(19)=.27, p=.794, d=0.06$, indicating the pitch effect was again driven by the rising pitch which produced speeded predicted motion.

Summary. I hypothesised that stimuli travelling along the horizontal plane accompanied by a rising pitch would elicit a premature TTC response (an underestimation), due to speeded predicted motion, and those accompanied by a falling pitch would elicit a delayed response (an overestimation) due to slowed predicted motion. Participants routinely overestimated the TTC of the visual stimulus, but in most conditions, a rising pitch produced faster predicted motion than a falling pitch. These effects were greatest at the far landmark position. Furthermore, the actual stimulus speed influenced the imagined stimulus speed. Compared to a constant pitch, the pitch effects were exaggerated for a rising pitch at the slow stimulus speed, and for a falling pitch at the fast stimulus speed. This indicates a slower developing but more robust effect for a rising pitch. The asymmetric effect of pitch is supported Eitan and Granot (2004), who found a rise in pitch produced increased speed judgements, however a decrease in pitch did not produce slowed speed judgements.

For stimuli travelling in the vertical plane, it was hypothesised that there may be gravity effects in addition to pitch effects, producing slower predicted motion for upward travelling stimuli and faster predicted motion for downward moving stimuli. The main effect for direction was significant in an analysis including all pitch conditions, but not when TTC estimates were transformed relative to silent TTC trials. This was not unexpected, as it suggests the pitch effects were of the same magnitude for upward and downward motion, and therefore that pitch and gravity have additive effects on predicted motion.

The aim of this experiment was first to determine whether pitch and direction of motion influenced predicted motion. The second aim was to identify which pitch parameters produce robust differences in TTC estimations. The difference between the TTC estimates for a rising and a falling pitch indicated a general effect of pitch. Further analyses compared each of these pitches to a constant pitch, indicating how pitch affects predicted motion. Clear effects of pitch were observed in both horizontal and vertical dimensions, consistent with a rising pitch speeding predicted motion, and a falling pitch slowing predicted motion. A direction effect was observed in the vertical, but not 
horizontal, condition, regardless of pitch. The direction effect is consistent with the activation of an internal model of gravity in the vertical condition (Zago \& Lacquaniti, 2005a). In both dimensions, pitch effects were moderated by object speed and landmark position. Overall, pitch effects were modulated by landmark position, showing the greatest effects at the far landmark position. In both stimulus planes, all stimulus speeds produced differences between a rising and falling pitch. However, relative to a constant pitch, the slow stimulus speed produced a rising pitch effect in which predicted motion was sped up, and the fast speed produced a falling pitch effect in which the predicted motion was slowed down. These diverging results indicate pitch is more likely to produce motion imagery biases when the stimulus speed produces conflicting information. In other words, when the speed and pitch information is congruent-for example a fast stimulus speed and a rising pitch - the pitch information is ignored as it does not conflict with the motion imagery. However, when the speed and pitch information is incongruent - fast stimulus speed and falling pitch - the auditory information is given more weighting as it is determined to be the more reliable, or stronger signal of the two (Welch \& Warren, 1980).

\section{Experiment 1B: Sudden Pitch Change at Occluder}

This thesis examines the influence the physical qualities of pitch have on the predicted motion of a moving visual stimulus. Experiment 1A presented pitch which featured a smooth transition from its starting frequency to its final frequency. This is similar to real world sound situations, for example the Doppler Effect, in which pitch changes dynamically as it approaches or recedes from an observer (Doppler; as cited in McBeath \& Neuhoff, 2002). However, it is interesting to determine what might happen if the pitch change did not occur gradually, for example if the pitch were to suddenly jump in frequency to one that is higher or lower. This would enable us to broaden our understanding of the factors that contribute to how well auditory and visual information is integrated in the brain. If the integration depends purely on the temporal aspect, then producing a pitch change at the time of visual occlusion should influence the visual percept (Wanatabe \& Shimojo, 2001). However, if audio-visual integration in a TTC task depends on the nature of the stimulus itself, the pitch will not be integrated with the visual information. Specifically, it may be that dynamic pitch change is needed to support audio-visual integration, as this mirrors real world situations. If dynamic change is needed, the pitch should not be integrated with the visual information, 
resulting in a breakdown of the seamless pairing of the auditory and visual stimuli, disrupting the pitch effects.

\section{Method}

\section{Participants}

Twenty people participated in this study ( 12 female; mean age 24.8 years). Participants were students or staff at Victoria University of Wellington and all had normal or corrected-to-normal vision and normal hearing. Seventeen were right handed. Approval was granted by the Human Ethics Committee of the School of Psychology, Victoria University of Wellington to carry out this experiment. Participants read an information sheet and gave written consent before experiment participation.

\section{Stimuli and Apparatus}

The apparatus and stimuli used in this experiment were identical to Experiment 1A, apart from the auditory stimuli. The frequency range of $500-400 \mathrm{~Hz}$ or $500-600 \mathrm{~Hz}$ was used as before (depending on the trial condition), but this time the pitch change was sudden rather than a gradual change. Again, the timing of the pitch change coincided with the moment the target reached the occluder. Although the participants were told to ignore the sound and judge the TTC on the visual stimulus alone, there was a concern that the participants might assume the stimulus had contacted the landmark point if the sound file terminated. To eliminate this possibility, the length of the sound file was changed to $5.7 \mathrm{~s}$ as opposed to a shorter $4.7 \mathrm{~s}$ length used in the prior experiment. The rate of pitch change was therefore $18 \mathrm{~Hz} / \mathrm{s}$.

\section{Procedure}

The procedure was the same as Experiment 1A.

\section{Results and Discussion}

Exclusion criteria remained the same as for Experiment 1A. Six participants met the criteria for exclusion in this experiment and were removed from the analysis. Of these, three participants did not respond within the correct time window on $10 \%$ or more of trials $(M=25.02 \%, S D=20.67 \%$, range $=13.39-48.89 \%)$. The remaining three participants had four or more trials deleted from one condition and were excluded from the analysis. Of the remaining 14 participants, an average of $1.43 \%$ of trials were deleted in the horizontal condition $(S D=2.26 \%$, range $=0-7.22 \%)$ and $2.74 \%$ in the vertical condition $(S D=3.87 \%$, range $=0-13.89 \%)$.

\section{Horizontal Trials}


TTC Performance: All Pitch Conditions. As with Experiment 1A, TTC deviations were first considered in the context of all pitch conditions, allowing the pattern of deviations compared to the actual TTC to be examined.

A 2 (stimulus direction: leftward, rightward) $\times 3$ (landmark position: near, middle, far) $\times 3$ (stimulus speed: slow, medium, fast) $\times 4$ (pitch condition: constant, rising, falling, silent) repeated measures ANOVA was used to compare deviation (in seconds) in TTC estimates.

All TTC estimates were overestimations (See table B1 in Appendix B). There was a main effect of pitch, $F(3,39)=3.24, p=.032, \eta p^{2}=.20$, in which accuracy was higher for constant and silent conditions $(M=0.34, S D=0.35$; and $M=0.31, S D=0.34$ respectively); rising $(M=0.35, S D=0.34)$, and a falling pitch both showed greater overestimations $(M=0.36, S D=0.34)$. Surprisingly, there was a main effect of stimulus direction, $F(1,13)=5.33, p=.038, \eta p^{2}=.29$, in which rightward stimuli produced lower overestimations $(M=0.32, S D=0.35)$ than leftward stimuli $(M=0.36$, $S D=0.33)$. There was an effect of stimulus speed, $F(1.11,14.47)=8.19, p=.011, \eta p^{2}$ $=.39$, in which greater overestimations were seen with increases in speed (slow $M=$ $0.25, S D=0.41$; medium $M=0.37, S D=0.32$; and fast $M=0.40, S D=0.29$ ).

There was no main effect of landmark position, $F(1.12,14.58)=0.29, p=.625$, $\eta p^{2}=.02$. There was however, a Landmark $\times$ Speed interaction, $F(1.50,19.43)=16.31$, $p<.001, \eta p^{2}=.56$. As seen in Figure 15, this interaction mirrored the pattern of results found in Experiment 1A, in which stimulus speed produced the largest TTC differences at the far landmark position. One-way repeated measures ANOVAs split by landmark showed no effect of speed at the near landmark, $F(2,26)=1.78, p=.189, \eta p^{2}=.12$, however there was at the middle, $F(1.18,15.34)=7.65, p=.011, \eta p^{2}=.37$, and far landmarks, $F(1.10,14.29)=11.92, p=.003, \eta p^{2}=.48$. At the middle landmark, a slow stimulus produced smaller TTC overestimations compared to medium, $t(13)=-4.10, p$ $=.002, d=-1.07$, and fast speeds, $t(13)=-2.78, p=.016, d=-0.74$, which did not differ from each other, $t(13)=-1.15, p=.271, d=-0.31$. At the far landmark, slow speed also produced significant underestimations compared to fast, $t(19)=-6.22, p<.001, d=-$ 1.39 , and medium speeds, $t(19)=-7.33, p<.001, d=-1.64$, and these differed from each other, $t(19)=2.64, p=.016, d=0.59$.

Finally, there was a Direction $\times$ Landmark interaction, $F(2,26)=3.60, p=.042$, $\eta p^{2}=.22$, in which there were larger overestimations for leftward compared to rightward motion at the middle and far landmarks. Paired-samples t-tests $(\alpha=0.05)$ 
were conducted to explore this interaction. As shown in Figure 16, there was no difference in TTC deviation between rightward and leftward moving stimuli at the near, $t(13)=-.17, p=.870, d=-0.04$, or middle landmarks, $t(13)=-1.63, p=.128, d=-0.43$. At the far landmark, rightward stimuli produced smaller TTC overestimations than leftward stimuli, $t(13)=-2.67, p=.019, d=-0.71$.

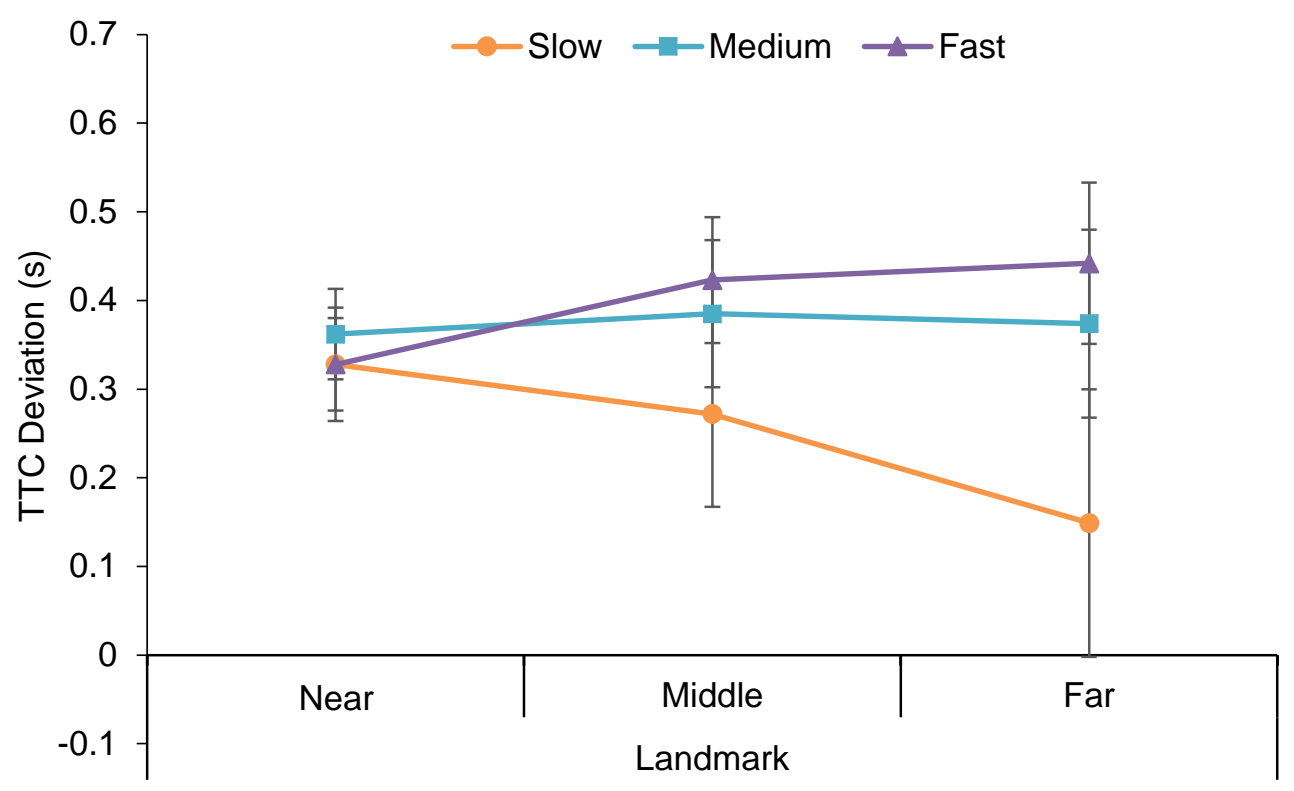

Figure 15. Deviations in TTC estimates for visual stimuli in slow, medium and fast speed conditions at the near, middle and far landmarks in Experiment 1B for horizontal trials. Error bars represent the standard error.

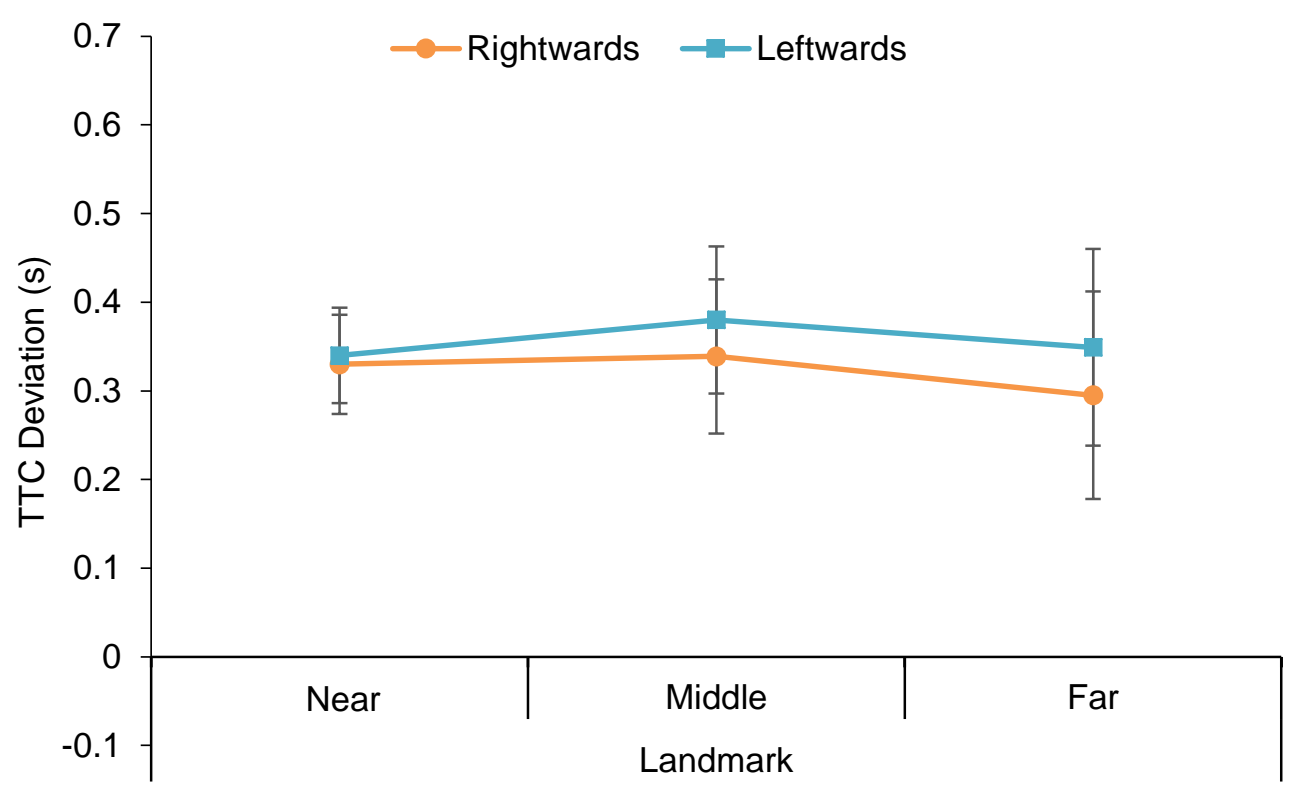

Figure 16. Deviations in TTC for visual stimuli by rightward or leftward motion, at near, middle and far landmark positions in Experiment 1B for horizontal trials. Error bars represent the standard error. 
TTC Performance: Relative to Silent Baseline. As with Experiment 1A, mean TTC deviations were overestimations (refer to Table B1 of the appendix), so the data was transformed in the same manner as Experiment 1A to produce TTC deviations relative to the silent condition. A 2 (stimulus direction: leftward, rightward) $\times 3$ (landmark position: near, middle, far) $\times 3$ (stimulus speed: slow, medium, fast) $\times 3$ (pitch condition: constant, rising, falling) repeated measures ANOVA was used to compare relative deviations in TTC estimates across conditions.

Interestingly, there was no main effect of pitch condition in this analysis, $F(1.42,18.46)=1.09, p=.350, \eta p^{2}=.24$. This result suggests that the sudden nature of the pitch change disrupted its pairing with the visual stimulus, preventing its integration in the TTC event. Support for this explanation stems from studies suggesting that if stimuli are similar enough the information will be processed in parallel; however, if stimuli are disparate, they will be interpreted as separate inputs and judged as two separate events (Freeman \& Driver, 2008; Körding et al., 2007; Maeda, Kanai, \& Shimojo, 2004; Schmiedchen et al., 2012; Shams, et al., 2002; Takeshima \& Gyoba, 2013; Watanabe \& Shimojo, 2001).

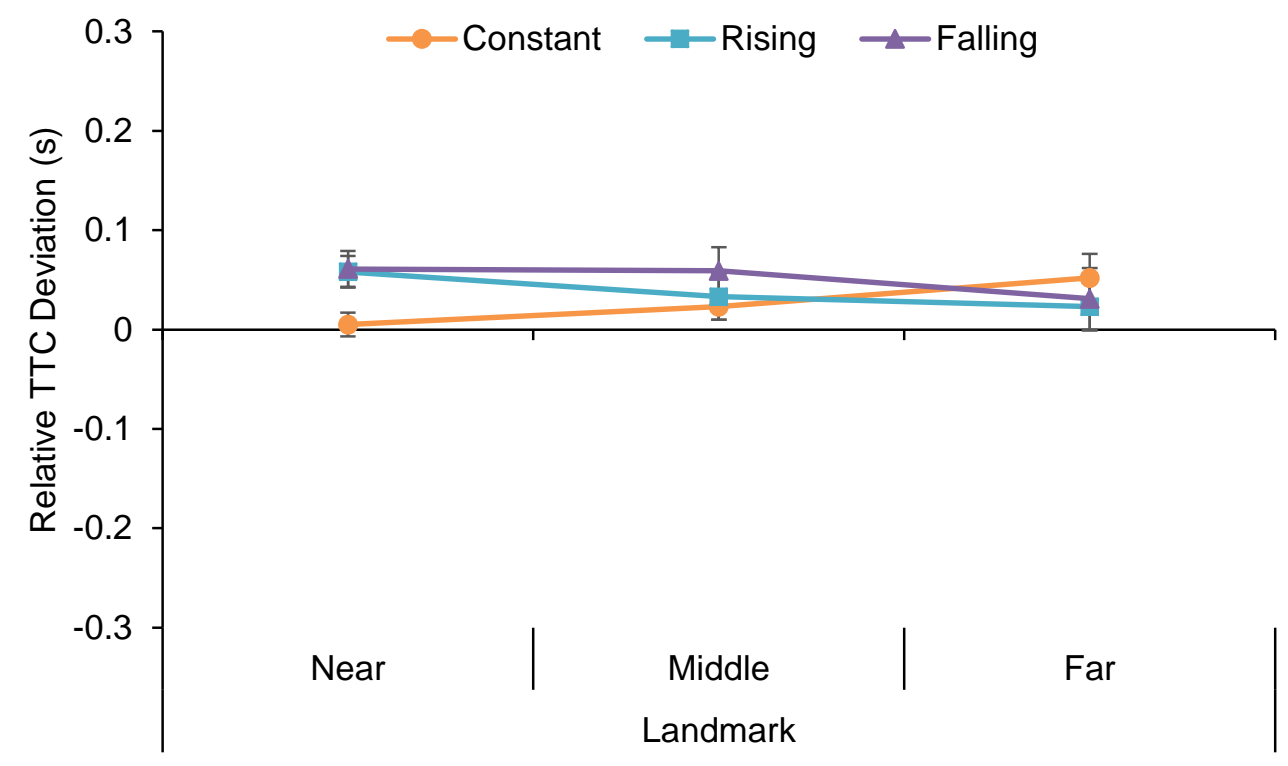

Figure 17. Relative deviations in TTC for visual stimuli in constant, rising and falling pitch conditions by near, middle and far landmark positions in Experiment 1B for horizontal trials. Error bars represent the standard error.

There was however a Landmark $\times$ Pitch interaction, $F(2.06,26.80)=3.89, p$ $=.032, \eta p^{2}=.23$, indicated in Figure 17, in which pitch effects were prominent at the near landmark. One-way repeated measures ANOVAs split by landmark indicated a main effect of pitch at the near landmark, $F(2,26)=11.89, p=.004, \eta p^{2}=.48$, but not 
at the middle, $F(2,26)=1.70, p=.202, \eta p^{2}=.12$, or far landmarks, $F(1.38,17.96)$ $=.60, p=.502, \eta p^{2}=.04$.

This is the opposite of the result observed in Experiment 1A. As Figure 17 indicates, a constant pitch produced underestimations relative to both rising, $t(13)=-$ $3.56, p=.004, d=-0.95$, and a falling pitch, $t(13)=-3.45, p=.004, d=-0.92$, which did not differ from each other, $t(13)=-.35, p=.734, d=-0.09$, suggesting a nonspecific effect of dynamic pitch. All other main effects and interactions were nonsignificant.

To summarise, in Experiment 1B, the horizontal condition showed very little in comparison to the horizontal trials of Experiment 1A. Specifically, there was no evidence of the predicted integration of pitch and speed, whereby a rising pitch should speed predicted motion, and a falling pitch should slow predicted motion. Contrary to hypotheses, there was a main effect of direction. Also, the interaction with landmark condition showed an effect of pitch at the near landmark, which was not consistent with the expected pitch effect. Specifically, the effect arose because both increases and decreases in pitch caused overestimations relative to a constant pitch. Furthermore, they did not differ from each other. It is clear that the nature of pitch change did not contribute to biases in predicted motion in this experiment.

\section{Vertical Trials}

TTC Performance: All Pitch Conditions. As with TTC performance in the horizontal plane, an ANOVA was first conducted including all pitch conditions. A 2 (stimulus direction: upward, downward) $\times 3$ (landmark position: near, middle, far) $\times 3$ (stimulus speed: slow, medium, fast) $\times 4$ (pitch condition: constant, rising, falling, silent) repeated measures ANOVA was used to compare TTC deviations (in seconds).

Again, all TTC estimates were overestimations ((See table B2 in Appendix B). There was a main effect of direction, $F(1,13)=13.51, p=.003, \eta p^{2}=.51$, in which downward moving stimuli $(M=0.27, S D=0.36)$ produced smaller overestimations than upward moving stimuli $(M=0.34, S D=0.36)$, again supporting the internal gravity hypothesis (Zago et al., 2010; Zago \& Lacquaniti, 2005a; Zago \& Lacquaniti, 2005b). 
As with Experiment 1A, there was no main effect of landmark, $F(1.18,15.35)$ $=.24, p=.674, \eta p^{2}=.02$. However, there was a Direction $\times$ Landmark interaction, $F(2$, 26) $=4.43, p=.022, \eta^{2}=.25$, as shown in Figure 18, in which downward moving stimuli produced smaller TTC deviations than upward moving stimuli at the near, $t(13)$ $=-2.96, p=.011, d=-0.79$, middle, $t(13)=-4.18, p=.001, d=-1.12$, and far landmarks, $t(13)=-2.40, p=.032, d=-0.64(\alpha=0.05)$. The gravity effect grew larger by landmark; however, so did TTC variance.

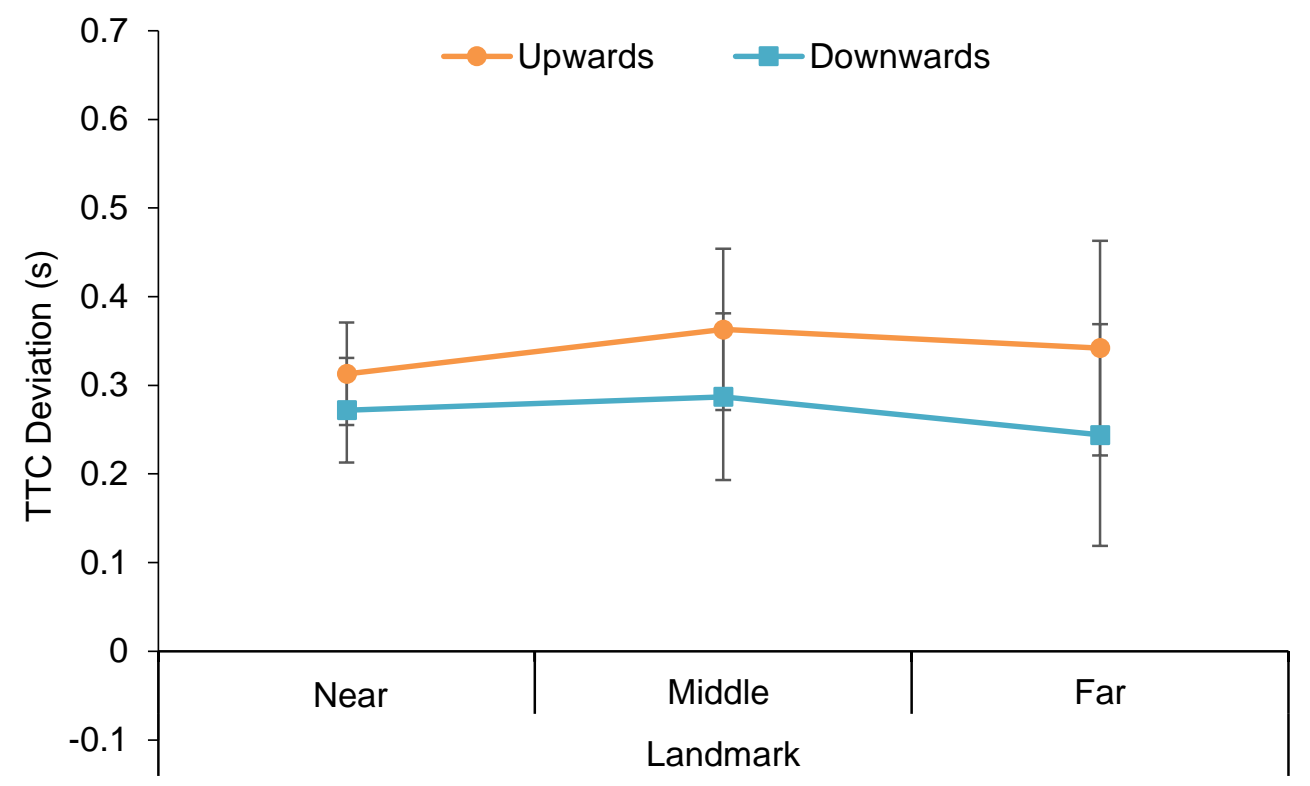

Figure 18. Deviation in TTC for upward and downward moving stimuli at the near, middle and far landmark positions in Experiment 1B for vertical trials. Error bars represent the standard error.

There was a main effect of pitch in this directional plane, $F(3,39)=3.80, p$ $=.018, \eta p^{2}=.23$, and a main effect of speed, $F(1.07,13.96)=10.58, p=.005, \eta p^{2}=.45$, contained within a Pitch $\times$ Speed interaction, $F(6,78)=3.01, p=.011, \eta p^{2}=.19$. Oneway repeated measures ANOVAs split by speed indicated no main effect of pitch at the slow stimulus speed, $F(3,39)=1.31, p=.285, \eta p^{2}=.09$, but effects of pitch at the medium, $F(3,39)=4.30, p=.010, \eta p^{2}=.25$, and fast stimulus speeds, $F(3,39)=5.04$, $p=.005, \eta p^{2}=.28$. As Figure 19 shows, there were no effects of pitch at the slow stimulus speed. At the medium speed, a rising and a falling pitch both produced greater overestimations than a constant pitch and silent trials, suggesting a general effect of pitch, however these differences did not reach statistical significance in paired-samples t-tests $(\alpha=.008 ; p \mathrm{~s}=.024$ or greater). At the fast stimulus speed, all the sound conditions produced larger overestimations than the silent condition, however the only 
statistical difference was between the falling and silent condition, $t(13)=3.27, p=.006$, $d=0.87(\alpha=.008$; all other $p s=0.24$ or greater $)$, indicating no pitch effect.

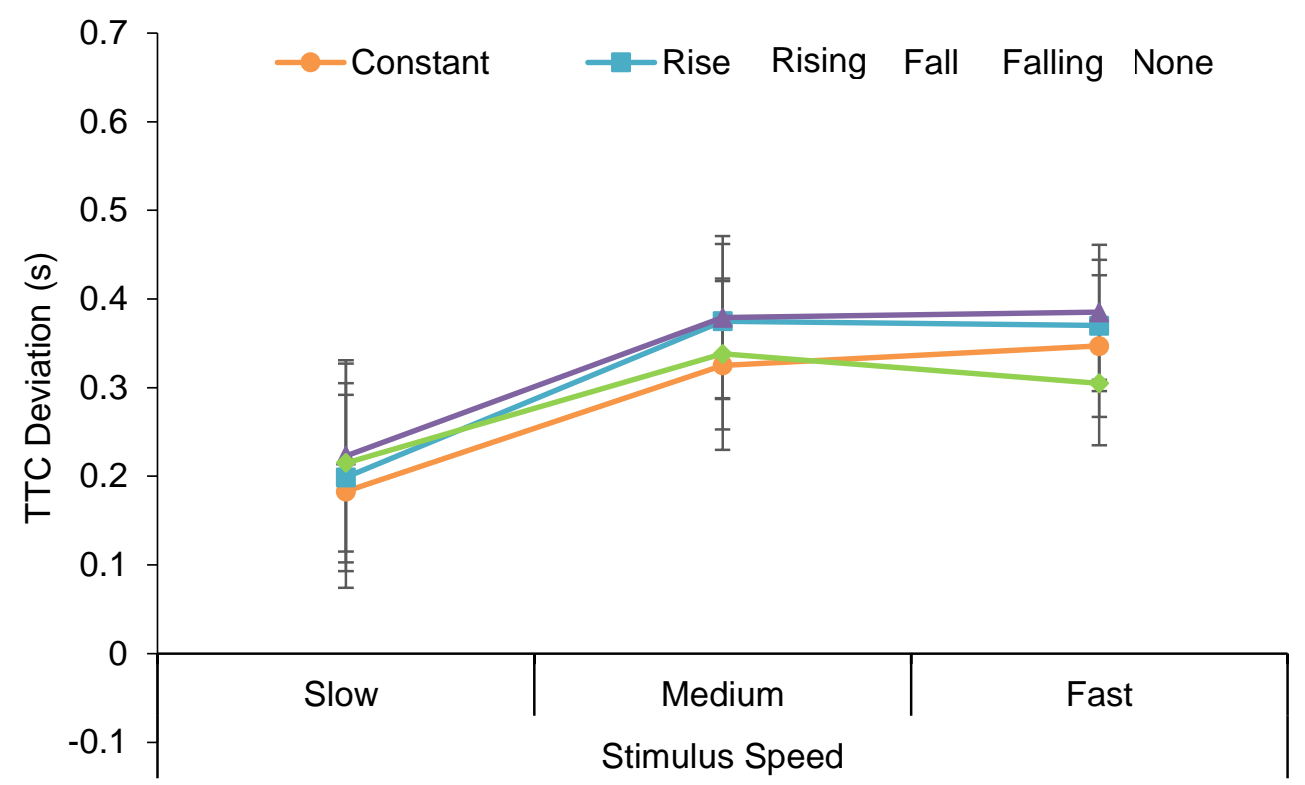

Figure 19. Deviations in TTC for visual stimuli in the constant, rising, falling, and silent conditions at slow, medium and fast stimulus speeds in Experiment 1B for vertical trials. Error bars represent the standard error.

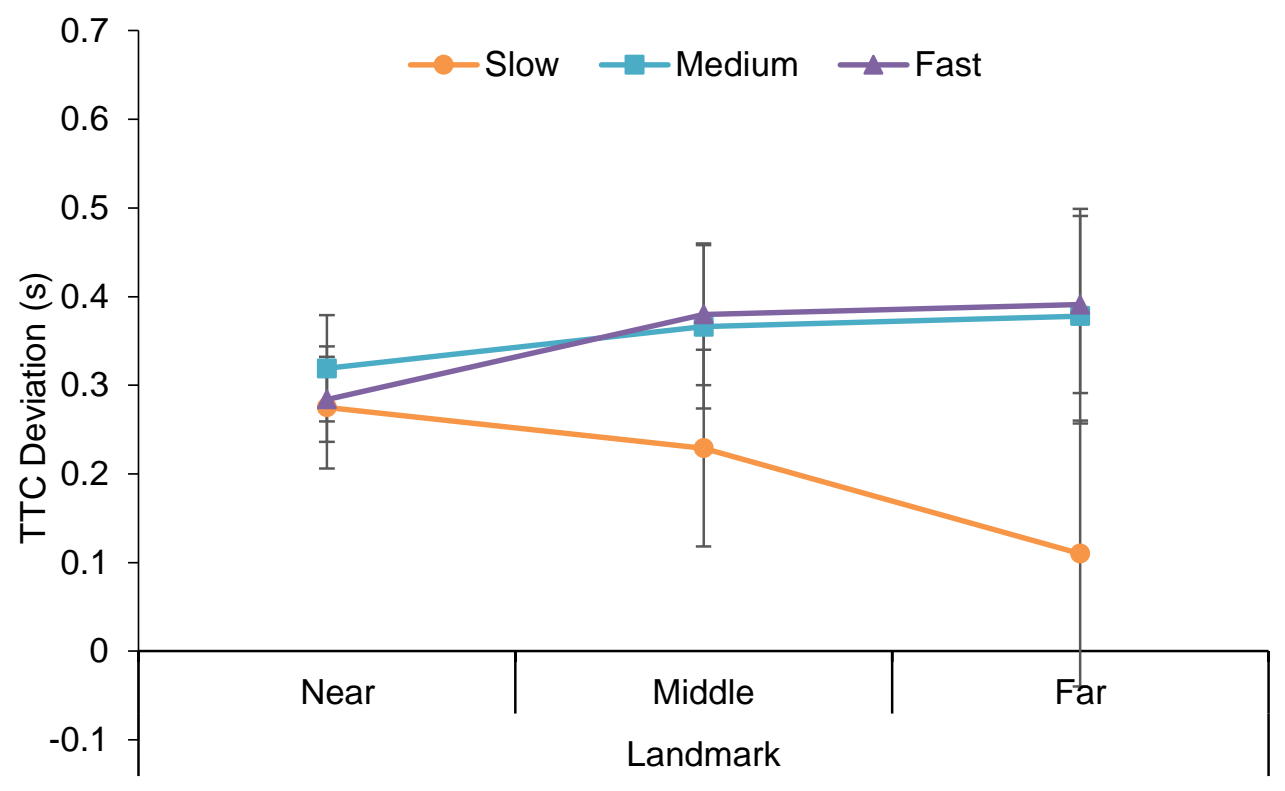

Figure 20. Deviations in TTC for visual stimuli in slow, medium and fast speed conditions at the near, middle and far landmarks in Experiment 1B for vertical trials. Error bars represent the standard error.

There was a Landmark $\times$ Speed interaction, $F(2.08,26.98)=27.47, p<.001, \eta p^{2}$ $=.68$, driven by smaller overestimations for slow stimuli at greater landmark positions. One-way ANOVAs split by landmark showed no effect of speed at the near landmark, $F(1.21,15.68)=1.72, p=.206, \eta p^{2}=.12$, but effects of speed at the middle, $F(1.12$, 
$14.37)=7.61, p=.013, \eta p^{2}=.37$, and far landmarks, $F(1.09,14.19)=20.11, p<.001$, $\eta p^{2}=.61$. As shown in Figure 20, there was no effect of speed at the near landmark. At the middle landmark, the slow speed produced significantly smaller overestimations than the medium, $t(13)=-3.92, p=.002, d=-1.05$, but not the fast speed, $t(13)=-2.57$, $p=.023, d=-0.69$, which did not differ from one another, $t(13)=-.52, p=.611, d=-$ 0.14. At the far landmark, a slow speed produced smaller overestimations in TTC compared to medium, $t(13)=-5.71, p<.001, d=-1.49$, and fast speeds, $t(13)=-4.14, p$ $=.001, d=-1.11$, which did not differ from one another, $t(13)=-.50, p=.626, d=-$ 0.13 .

TTC Performance: Relative to Silent Baseline. As with the prior analyses, mean TTC deviations were positive indicating overestimations overall. The data was transformed to produce TTC deviations relative to the silent condition. Positive values indicate relative overestimations, suggesting slowed predicted motion, and negative values indicate underestimations, suggesting speeded predicted motion.

A 2 (stimulus direction: upward, downward) $\times 3$ (landmark position: near, middle, far) $\times 3$ (stimulus speed: slow, medium, fast) $\times 3$ (pitch condition: constant, rising, falling) repeated measures ANOVA was used to determine relative TTC deviations (in seconds).

There was a main effect of pitch, $F(2,26)=3.99, p=.031, \eta p^{2}=.24$, such that the constant pitch condition produced TTC estimates equivalent to silent trials $(M=$ $0.00, S D=0.14)$; the rising pitch condition produced overestimations $(M=0.03, S D=$ $0.15)$, and the falling pitch condition even more so $(M=0.04, S D=0.16)$, indicating these conditions both slowed predicted motion. This result does not reflect the predicted pitch effect which was observed in Experiment 1A; underestimations for a rising pitch and overestimations for a falling pitch.

There was a main effect of stimulus speed, $F(2,26)=7.30, p=.003, \eta p^{2}=.36$, such that there were underestimations at the slow stimulus speed $(M=-0.01, S D=$ $0.16)$, and overestimations at the medium $(M=0.02, S D=0.15)$ and fast stimulus speeds $(M=0.06, S D=0.15)$. All other main effects and interactions were nonsignificant.

Summary. In the horizontal trials, there was no main effect of pitch in the second analysis, indicating that pitch did not affect motion prediction as expected. Instead, findings suggest that the sudden nature of the pitch change disrupted the integration of the sound and visual stimulus (Körding et al., 2007; Schmiedchen et al., 
2012). Furthermore, the pitch effects were only evident at the near landmark position, and the interaction was driven by the constant pitch condition, differing greatly from the results in Experiment 1A.

In the vertical condition, there was a main effect of pitch, in which both a falling and a rising pitch produced overestimations, indicating slowed predicted motion. Again, this result is inconsistent with predictions and findings of Experiment 1A, in which a rising pitch produced faster predicted motion. Furthermore, interactions that were found were evident only in the first analysis which included the silent condition, suggesting they affected all stimuli regardless of pitch.

Speed moderated pitch much differently compared to Experiment 1A; the greatest pitch effects were found at the fast stimulus speed, and the slow speed resulted in no main effect of pitch. As with Experiment 1A, the gravity effect was only found when all pitches (including silent) were included in the analysis, and the effect was greatest at the far landmark, as in Experiment 1A. This result indicates the gravity effect is robust and that it is entirely separate from the pitch effect, as it is not affected by pitch manipulations.

\section{Experiment 2: Influence of TMS to the STS on Predicted Motion}

Experiments $1 \mathrm{~A}$ and $1 \mathrm{~B}$ were conducted to first determine whether pitch and gravity affect TTC estimates. The second aim was to find the experimental parameters which produced the most pronounced effects. Experiment 1A used gradual descending and ascending pitch, finding they altered predicted motion as expected. However, Experiment 1B used sudden pitch changes, which did not alter motion judgements. The conflicting results for Experiments $1 \mathrm{~A}$ and $1 \mathrm{~B}$ indicate that for pitch changes to affect visual motion imagery, they must occur when the visual stimulus is under occlusion, and these changes must be gradual in order to be processed as part of the TTC experience. Based on these results, I used the gradual ascending and descending pitch in Experiment 2. Furthermore, in order to investigate a potential dissociation between pitch and gravity effects, Experiment 2 used vertical motion only.

The far landmark was chosen because both pitch and gravity effects were largest at this landmark in Experiment 1A. The investigations revealed stimulus speed was important in modulating the type of pitch-effects exhibited. In Experiment 1A, a rising pitch sped up predicted motion when the stimulus was moving at a slow pace, and a falling pitch slowed predicted motion when the stimulus was moving quickly. However gravity effects seemed to be slightly greater at the slow stimulus speed. Therefore, 
Experiment 2 examined TTC using only one speed — formerly the slow stimulus speed - to enable examination of both pitch and gravity effects. Due to choice of the slow stimulus speed, it is possible Experiment 2 will produce larger effects for a rising pitch than for a falling pitch, in comparison to a constant pitch.

Experiment 2 goes a step further than Experiment 1A; it not only seeks to replicate the results using the parameters which elicit the largest pitch effects, but also determine the cortical site for the audio-visual integration of visual motion and pitchspeed effects. Both the left and right STS were the target sites of interest, because previous studies have found these to be involved in audio-visual binding in other domains (Calvert et al., 2001; Beauchamp et al., 2010; Hocking \& Price, 2008).

Experiment 2 utilised TMS, a technique that is used to infer causality (Sandrini et al., 2011). There are multiple TMS techniques to disrupt or enhance neural activity depending on the desired outcome and task demands. Single pulse TMS is useful in determining the temporal recruitment of certain brain areas in a task (Sandrini et al., 2011). A train of consecutive single pulses is termed repetitive TMS (rTMS; Sandrini et al., 2011). This method produces an extended period of disruption, and is therefore useful for tasks in which brain areas are recruited over longer periods of time. For example, rTMS at low frequency $(\leq 1 \mathrm{~Hz})$ over the posterior region of the STS impairs the perception of biological motion (Grossman et al., 2005). Furthermore, rTMS can be delivered online or offline (Sandrini et al., 2011). Online rTMS is applied while participants complete an experimental task; however this is not always practical. When stimulation at a site causes facial twitches that interfere with task performance, rTMS is applied offline before a task (Sandrini et al., 2011). Repetitive TMS can also be delivered in different patterns of stimulation, depending on the desired outcome.

I used theta burst stimulation, a sequence in which three bursts of pulses are delivered at a $5 \mathrm{~Hz}$ interval at a repeating frequency of $50 \mathrm{~Hz}$ (TBS; Huang, Edwards, Rounis, Bhatia, \& Rothwell, 2005; Oberman, Edwards, Eldaief, \& Pascual-Leone, 2011). There are two types of TBS which alter motor evoked potentials (MEPs). In Intermittent TBS, a 2 second train of these pulses are delivered every 10 seconds for 190 seconds total; this results in behavioural facilitation for up to 15 minutes (Huang et al., 2005). This facilitation in behaviour is indicated by lower thresholds of stimulation needed to elicit a motor response (Huang et al., 2005). The alternative, continuous TBS (cTBS) consists of a train of uninterrupted pulses, given for forty seconds; this causes inhibition of MEPs for up to an hour (Huang et al., 2005). 
An advantage of TBS paradigms is that shorter periods of stimulation are required compared to rTMS; 600 pulses of TBS takes 40-190 seconds, whereas 600 pulses of rTMS takes 10 minutes to deliver (Wu, Shahana, Huddleston, \& Gilbert, 2012). Furthermore, a modified TBS paradigm can be applied using a frequency of $30 \mathrm{~Hz}$ instead of $50 \mathrm{~Hz}$, without reducing the time course, or strength of the behavioural effects (Tsang, Jacobs, Lee, Asmussen, Zapallow, \& Nelson, 2014; Wu et al., 2012). Lower frequency stimulation produces less heating of the coil-making it more comfortable for the participant — and a lower chance of inducing seizures (Wu et al., 2012).

Therefore, Experiment 2 was a pilot study which used a modified $30 \mathrm{~Hz}$ cTBS paradigm (Wu et al., 2012) to determine whether the STS is responsible for binding the visual and auditory (pitch-speed) components found to influence predicted motion in Experiment 1A. Both the left (Beauchamp et al., 2004; Beauchamp et al., 2010; James, VanDerKlok, Stevenson, \& James, 2011; Liebenthal, Desai, Humphries, Sabri, \& Desai, 2014; Werner \& Noppeney, 2009) and right (Bischoff et al., 2007; Calvert, Campbell, \& Brammer, 2000; Werner \& Noppeney, 2009) STS have been implicated in audio-visual integration. Inhibitory effects on the cortex are expected, meaning if the STS is responsible for integrating the audio-visual TTC information, stimulation of both the left and the right sites should disrupt this effect. Disruption in this area will be characterised by no change in TTC estimates with the presentation of a rising and a falling pitch compared to a constant pitch. However, the gravity effect should not be affected by TBS as such effects are postulated to have neural substrates in the vestibular network, involving regions such as the cingulate gyrus, inferior frontal gyrus and supramarginal gyrus (see Zago \& Lacquaniti, 2005b for a review).

\section{Method}

\section{Participants}

Participants were 14 (eight female; mean age 28.86 years) adults; all were current or former students or staff at Victoria University of Wellington. All had normal or corrected-to-normal vision and normal hearing. Thirteen were right handed. All participants met the inclusion criteria: no family history of epilepsy, neurological or psychiatric disorders, history of headaches or frequent fainting spells; not currently on neuron-altering medication, no previous concussions, and no metal in the head. Approval was granted by the Human Ethics Committee of the School of Psychology, Victoria University of Wellington to carry out this experiment. Participants read an 
information sheet (including information about exclusion criteria) and gave written consent before experiment participation.

\section{Stimuli and Apparatus}

As with Experiments $1 \mathrm{~A}$ and $1 \mathrm{~B}$, stimuli were presented on a Samsung $51 \mathrm{~cm} \times$ $29 \mathrm{~cm}$ monitor with a refresh rate of $120 \mathrm{~Hz}$ (run through a Dell PC). The experiment ran on a 64 bit operating system equipped with Windows 7, and was constructed using Psychopy (Peirce, 2007) version 1.80.03. Head movements were minimised by using a full-head chin-rest, positioned $57 \mathrm{~cm}$ from the screen.

As in Experiment $1 \mathrm{~A}$, dot stimuli were $1 \mathrm{~cm}$ in diameter, pertaining to $1^{\circ}$ of visual angle. These stimuli travelled at a speed of $5.5 \mathrm{~cm} / \mathrm{s}$. The visual stimuli travelled within a $27 \mathrm{~cm}$ long track, positioned at the centre of the screen, and a $17 \mathrm{~cm} \times 1 \mathrm{~cm}$ visual occluder was positioned $5 \mathrm{~cm}$ from the screen's centre. The landmark was positioned $9.25 \mathrm{~cm}$ from the centre of the screen $(12.75 \mathrm{~cm}$ along the occluder). On catch trials, landmarks were presented at 8.25 or $10.25 \mathrm{~cm}$ from the centre of the screen (11.75 and $13.75 \mathrm{~cm}$ respectively). Auditory tones were created using Audacity version 2.0.5 (Audacity, 2008). The constant sound stimulus was $500 \mathrm{~Hz}$, and $5.7 \mathrm{~s}$ in duration. On dynamic sound trials, at 1.72 seconds the sound changed from $500 \mathrm{~Hz}$ and either steadily increased in frequency (rising sound condition) or fell in frequency (falling sound condition) by $18 \mathrm{~Hz} / \mathrm{s}$. Auditory stimuli were played through Phillips over-ear circumaural headphones.

\section{Brain Site Localisation}

Prior to experiment participation, a $T_{1}$-weighted high resolution magnetic resonance imaging (MRI) brain scan was obtained for each participant at the Wellington Hospital, using a 3T scanner (Philips, Medical Systems, Bothell, USA). MRI scans were then used to create 3D reconstructions of each participant's brain using Brainsight 2, a frameless stereotaxic neuronavigation system (BrainSight 2; Rogue Research, Canada), allowing the position of the TMS coil to be tracked in real time.

Participants were fitted with an electrodeless electroencephalogram (EEG) cap, configured in the 10-20 coordinate system. Cap size was determined by a measurement around the circumference of the head, from the middle of the forehead, around past the inion, and positioned so that the midpoint $(\mathrm{Cz})$ sat between the nasion and the inion, and the preaurical notches of both ears. An elastic headband with three passive markers was attached to the participant's head, which allowed registration of the participant's head in 3-dimensional space. The tip of the nose, nasion, and left and right preauricular 
notches were used as landmarks to register the participant's head in space, by using a pointer equipped with passive markers.

The left and right STS were localised via a combination of Talairach coordinates (Talairach \& Tournoux, 1988) and anatomical location (see Figure 21 for STS location). Talairach coordinates were taken from a meta-analysis produced by Hein and Knight (2008), including five audio-visual integration studies that targeted the STS, and were converted into MNI space (Montreal Neurological Institute); see Table 2.

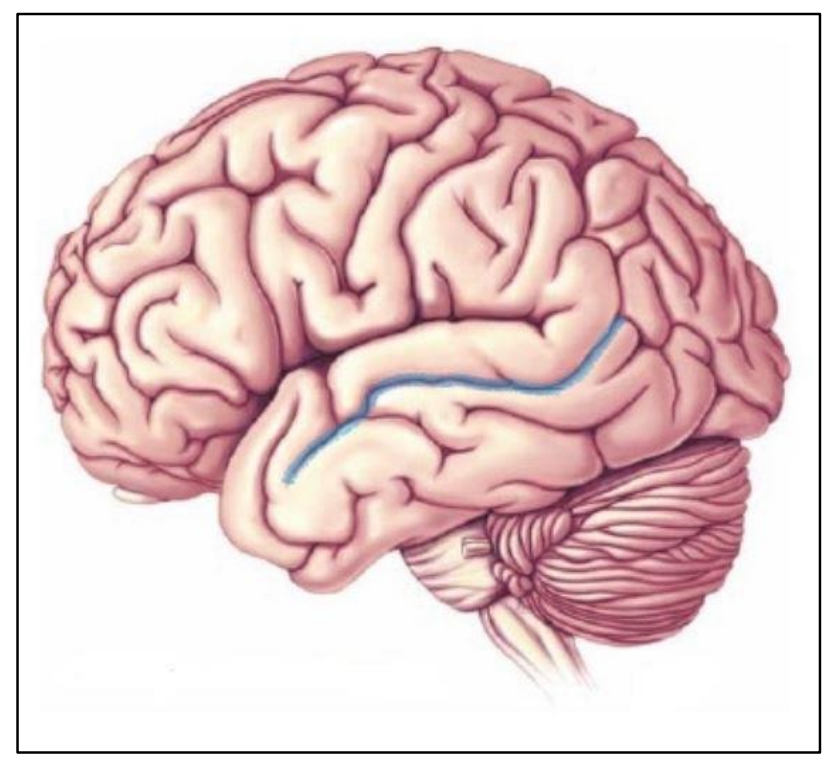

Figure 21. Lateral view of the cerebrum showing the left superior temporal sulcus (in blue). Adapted from Bear, Connors and Paradiso (2006).

Table 2

Original Talairach coordinates from Hein and Knight (2008), transformed into MNI coordinates for Experiment 2.

\begin{tabular}{lccc}
\hline \multirow{2}{*}{ Stimulation Site } & \multicolumn{3}{c}{ Talairach Coordinates } \\
\cline { 2 - 4 } Left pSTS & -49.70 & $y$ & 14.30 \\
Right pSTS & 49.90 & -55.40 & 12.80 \\
\hline & $x$ & -49.30 & $z$ \\
\cline { 2 - 4 } & \multicolumn{4}{c}{ MNI Coordinates } & 17.21 \\
\hline Left pSTS & -51.69 & -56.51 & 13.73 \\
Right pSTS & 54.70 & -49.55 & \\
\hline
\end{tabular}


Table 3

MNI coordinates by participant $(\mathrm{mm})$ for the two target sites: the left and right STS, and motor threshold (MT) and intensity of TBS stimulation in Experiment 2.

\section{MNI Coordinates}

\begin{tabular}{|c|c|c|c|c|c|c|c|c|}
\hline & & Left $\mathrm{S}$ & & & Right ST & & TBS & rameters \\
\hline Participant & $x$ & $y$ & $z$ & $x$ & $y$ & $z$ & MT & Intensity \\
\hline 1 & -52.09 & -57.71 & 15.66 & 54.70 & -49.55 & 13.73 & 58 & $44 \%$ \\
\hline 2 & -56.59 & -53.32 & 16.19 & 55.20 & -48.10 & 17.64 & 69 & $51 \%$ \\
\hline 3 & -56.91 & -58.09 & 20.06 & 61.19 & -50.57 & 17.33 & 68 & $51 \%$ \\
\hline 4 & -55.43 & -58.62 & 14.29 & 56.15 & -45.87 & 11.38 & $>70$ & $51 \%$ \\
\hline $5^{a}$ & -51.64 & -53.31 & 19.75 & 56.55 & -47.04 & 18.14 & 69 & $51 \%$ \\
\hline $6^{\mathrm{a}}$ & -52.46 & -62.03 & 6.51 & 57.46 & -48.88 & 18.93 & 69 & $51 \%$ \\
\hline 7 & -49.23 & -55.20 & 16.76 & 58.31 & -46.80 & 4.48 & 61 & $46 \%$ \\
\hline 8 & -51.39 & -57.89 & 7.22 & 57.12 & -46.74 & 13.22 & 66 & $49 \%$ \\
\hline 9 & -51.69 & -56.51 & 17.21 & 55.86 & -50.66 & 11.18 & 61 & $46 \%$ \\
\hline 10 & -52.58 & -64.74 & 10.35 & 59.19 & -53.57 & 8.81 & 65 & $49 \%$ \\
\hline 11 & -57.75 & -54.95 & 11.81 & 61.21 & -50.30 & 9.19 & 63 & $48 \%$ \\
\hline 12 & -55.65 & -57.49 & 13.07 & 56.95 & -51.22 & 5.08 & 53 & $40 \%$ \\
\hline 13 & -54.46 & -51.24 & 16.08 & 50.06 & -51.74 & 13.51 & $>70$ & $51 \%$ \\
\hline 14 & -58.24 & -53.74 & 17.55 & 60.05 & -52.12 & 9.63 & 69 & $51 \%$ \\
\hline
\end{tabular}

Note. Participants with motor thresholds of 69 or higher were stimulated at maximum stimulator output (51\%), as supported by Bertini, Avenanti, \& Ladavas (2010).

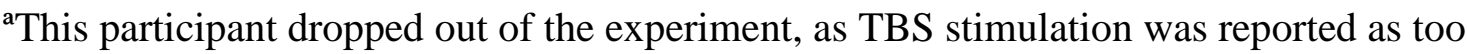
intense. 
The transformed MNI coordinates formed the basis for localisation of the left and right STS. If the coordinates did not lie on the sulcus, the visual marker was moved accordingly. See Table 3 for individual coordinates used. The vertex was used as a control site for the non-specific effects of the TBS stimulation and to ensure that any changes in behavioural results were due to the TBS stimulation and not external factors. A white sticker was placed on EEG site $\mathrm{Cz}$ on the participants head, and the neuronavigator was used to ensure stimulation was delivered to the sulcus between the left and right hemispheres.

\section{TBS Protocol}

Cortical stimulation was delivered via a $70 \mathrm{~mm}$ figure 8 coil using a biphasic stimulator, held in place with a clamp on an extendable tripod stand. Continuous thetaburst stimulation (cTBS) was applied using a modified protocol adapted from $\mathrm{Wu}$ et al. (2012). The cTBS consisted of 200 bursts of three pulses delivered at $30 \mathrm{~Hz}$, repeated at $5 \mathrm{~Hz}$, for a total of 600 pulses delivered over 40s. Stimulation intensity was set at $75 \%$ of the individual resting motor threshold. Recent cTBS studies targeting the STS have used $80 \%$ of participants' active motor threshold (Murakami, Kell, Restle, Ugawa, \& Ziemann, 2015; Tarnutzer, Lasker, \& Zee., 2013), and cTBS studies targeting other cortical areas commonly use $70 \%$ of the individual's resting motor threshold (Gentner, Wankerl, Reinsberger, Zeller, \& Classen., 2008; Goldsworthy, Pitcher, \& Ridding., 2013; Tsang et al., 2014). Therefore, $75 \%$ was chosen for the current study, as it falls midway between commonly used intensities in the cTBS literature, and allowed for stimulation of participants with a motor threshold of up to 68 (maximum stimulator output was 51). Participants with a motor threshold of 69+ were stimulated at maximum stimulator output (Bertini et al., 2010).

Motor thresholds were established by fitting an EEG cap as described earlier, and single pulses were delivered to the $\mathrm{C} 3$ site at $65 \%$ of the stimulator output. The intensity was increased or decreased by $2 \mathrm{~Hz}$ at a time to find the intensity which elicited visible twitches in the forefinger or thumb in the right hand on five out of 10 occasions (Pridmore, as cited in Westin, Bassi, Lisanby, \& Luber, 2014). Often the participant's hand area was not directly under site C3; in this case, the scalp was targeted in a grid like formation until twitches were elicited. The coil orientation was held constant for motor threshold between participants, with the handle pointing laterally and wings horizontal. 
The motor threshold was determined for each participant at the beginning of the first of their four sessions. All participants completed a TMS screening form prior to experiment participation, and TMS safety guidelines were strictly adhered to. On occasion, participants experienced minor, temporary headaches at the site of cTBS stimulation; no other side effects were reported.

\section{Procedure}

Each participant completed four experimental sessions on separate days; cTBS to the left pSTS, right pSTS, vertex, and a no TMS control condition. The coil was oriented such that it was tangential to the scalp with the handle pointing backwards towards the occiput. In some instances, the wings of the coil were touching the edges of the ear; in these cases the coil was moved until the participant reported that it was no longer touching (Cattaneo, Sandrini, \& Schwarzbach, 2010). At the start of each cTBS session, participants were fitted with a cap and seated in front of the experimental computer, within view of the Polaris (a camera used for neuronavigation). Following registration of the participant's head in 3-dimensional space, the TMS coil was positioned in the correct cortical location, guided by the neuronavigator in real time. The coil position deviated no more than $2 \mathrm{~mm}$ from the intended stimulation site. Participants were provided with earplugs and were seated with their head in a chinrest for 40 seconds of stimulation. Continuous TBS stimulation was followed by a five minute break before commencement of the experiment to allow time for the effects to reach their peak (Huang et al., 2005).

The paradigm was similar to that of Experiment 1A. Participants were seated in the same room as the prior experiments, with their heads secured in a chin rest. Stimulus motion was always vertical, and there was a single landmark position (previously the 'far' landmark location), with the exception of catch trials. Therefore, the dot moved upward or downward until it reached the landmark position, and participants responded using the spacebar when they judged the dot to have contacted the landmark, shown in Figure 22. 
As with the previous experiments, participants were informed there would sometimes be sound trials, but were instructed to ignore the sound and base their judgements on the visual stimulus alone. Unlike Experiments $1 \mathrm{~A}$ and 1B, the stimulus motion started automatically. This was signalled by the white dot briefly turning green. This manipulation held the total experiment time constant across participants; in the previous experiments participants controlled when the stimulus motion started, meaning it was possible to take unscheduled breaks between trials. Because TMS effects fade with time, it was important that all participants completed the experimental task within the same time window.

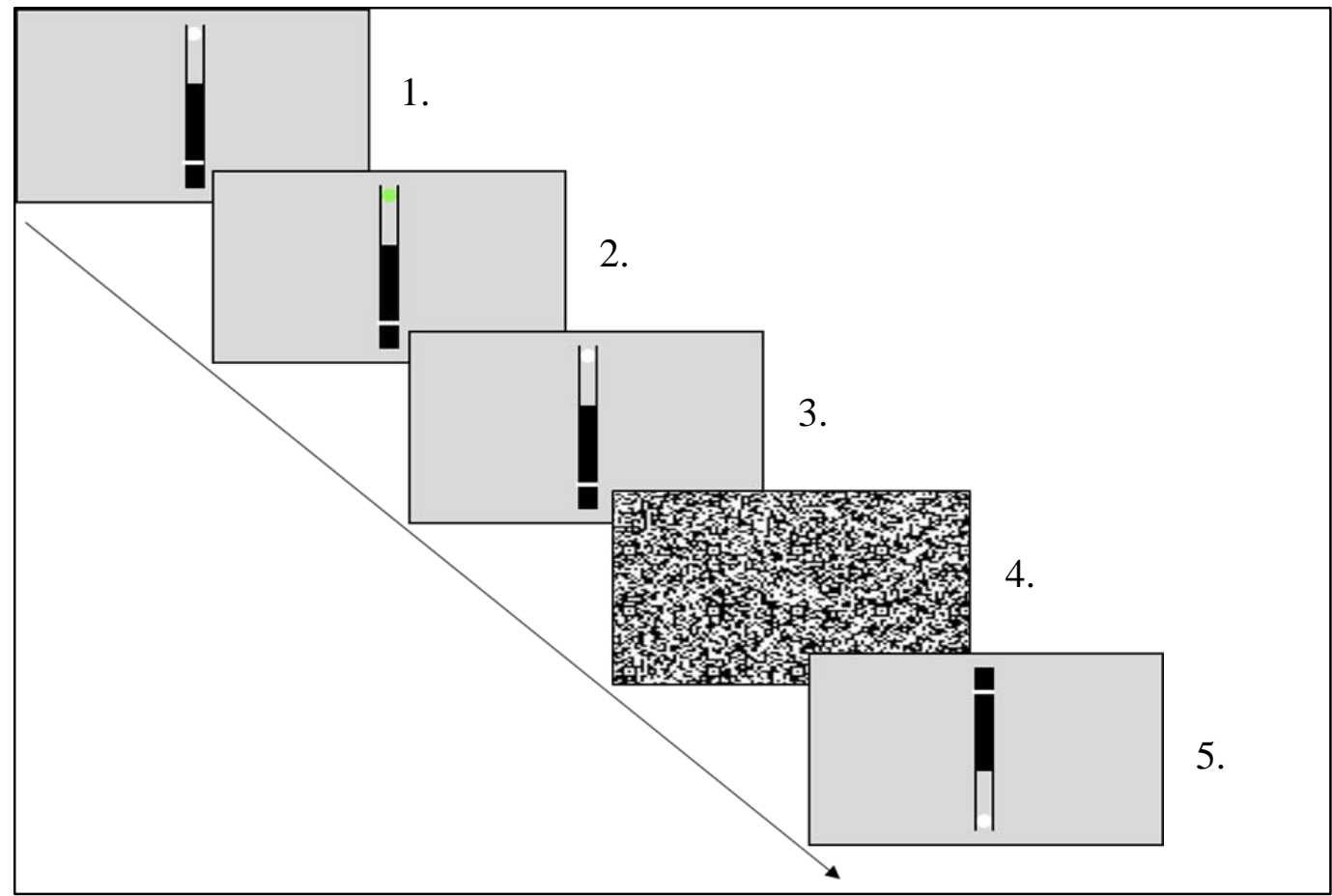

Figure 22. Trial procedure in Experiment 2. White dot flashes green briefly before moving in a straight line of motion behind the occluder. Participants press the spacebar key when they estimate the white dot has reached the landmark (white line). Stimuli are not to scale.

Sequence of events:

1. White dot waits at one end of a track for $0.5 \mathrm{~s}$.

2. Dot flashes green for $0.5 \mathrm{~s}$ to signal trial is about to start.

3. After $0.5 \mathrm{~s}$, white dot moves to opposite end of track. $500 \mathrm{~Hz}$ tone paired with dot, changes at occluder: no change/rising or falling by $18 \mathrm{~Hz} / \mathrm{s}$.

4. Visual mask presented for $0.5 \mathrm{~s}$.

5. New randomised trial appears.

Prior to the experiment, participants were presented with 10 silent practice trials which did not include feedback. The experiment included 180 trials in total, 20 of which were catch trials. In these catch trials, the landmark position was staggered forwards or backwards by $1 \mathrm{~cm}$, to check task attention. The experiment contained 2 
stimulus directions [upward, downward] $\times 4$ pitch conditions [constant, rising, falling, none]). There were 20 repetitions of each condition, and 4 breaks interspersed throughout the experiment. TMS session order was partially counterbalanced between participants, determined by a Latin square design: $50 \%$ of block orders featured the target sites in consecutive sessions (e.g. vertex, left STS, right STS, no TMS) and the remainder had stimulation of target sites separated by control sites. Experimental conditions were fully randomised within subjects.

\section{Results}

As with Experiments 1A and 1B, TTC error values (TTCe), were calculated from raw observed TTC values, in which the actual TTC (TTCa) was taken away from the observed TTC (TTCo). Positive values indicate overestimations in seconds (where the visual stimulus is estimated to be moving slower than it actually is [TTCo > TTCa]) and negative values indicate underestimations (the visual stimulus is estimated to be moving faster than it actually is [TTCo $<$ TTCa]).

Trials in which participants made a response 2 seconds before or after the actual TTC were deemed to be indicative of distraction from the task and were removed from the analysis ${ }^{2}$. Furthermore, participants whom did not show different TTC values for catch trials were removed from the analysis. All other criteria was the same as Experiments 1A and 1B. No participants were excluded on the basis of these criteria. However, two participants dropped out of the study due to finding the TBS stimulation aversive (Participants five and six in Table 3). Analyses are therefore based on the remaining 12 participants. The average rate of rejection was $2.27 \%$ of trials $(S D=$ $2.48 \%$, range $=0-15 \%)$.

\section{TTC Performance: All Pitch Conditions}

Baseline Performance. In order to determine how TTC performance in TMS conditions compared to baseline, performance was first examined in the no TMS condition, and qualitatively compared to the comparable conditions of Experiment $1 \mathrm{~A}$ (that is, judgements of the slow-moving object at the far landmark position).

\footnotetext{
${ }^{2}$ In this experiment, $2 \mathrm{~s}$ was used as participant responses deviated further from the actual TTC than for Experiments $1 \mathrm{~A}$ and $1 \mathrm{~B}$.
} 
Visual inspection of the data in Figure 23 indicates overestimations overall (See table $\mathrm{C} 1$ in Appendix $\mathrm{C}$ ); this is unsurprising given the behavioural results in Experiments $1 \mathrm{~A}$ and 1B. However, there appears to be no gravity effect, as performance remains relatively unchanged in downward versus upward trials. A rising pitch produced lower overestimations compared to the other conditions, suggesting speeded predicted motion. There appear to be no significant difference between a falling pitch and silent trials, which is expected due to the relationship between slow speed and a rising pitch but not a falling pitch.

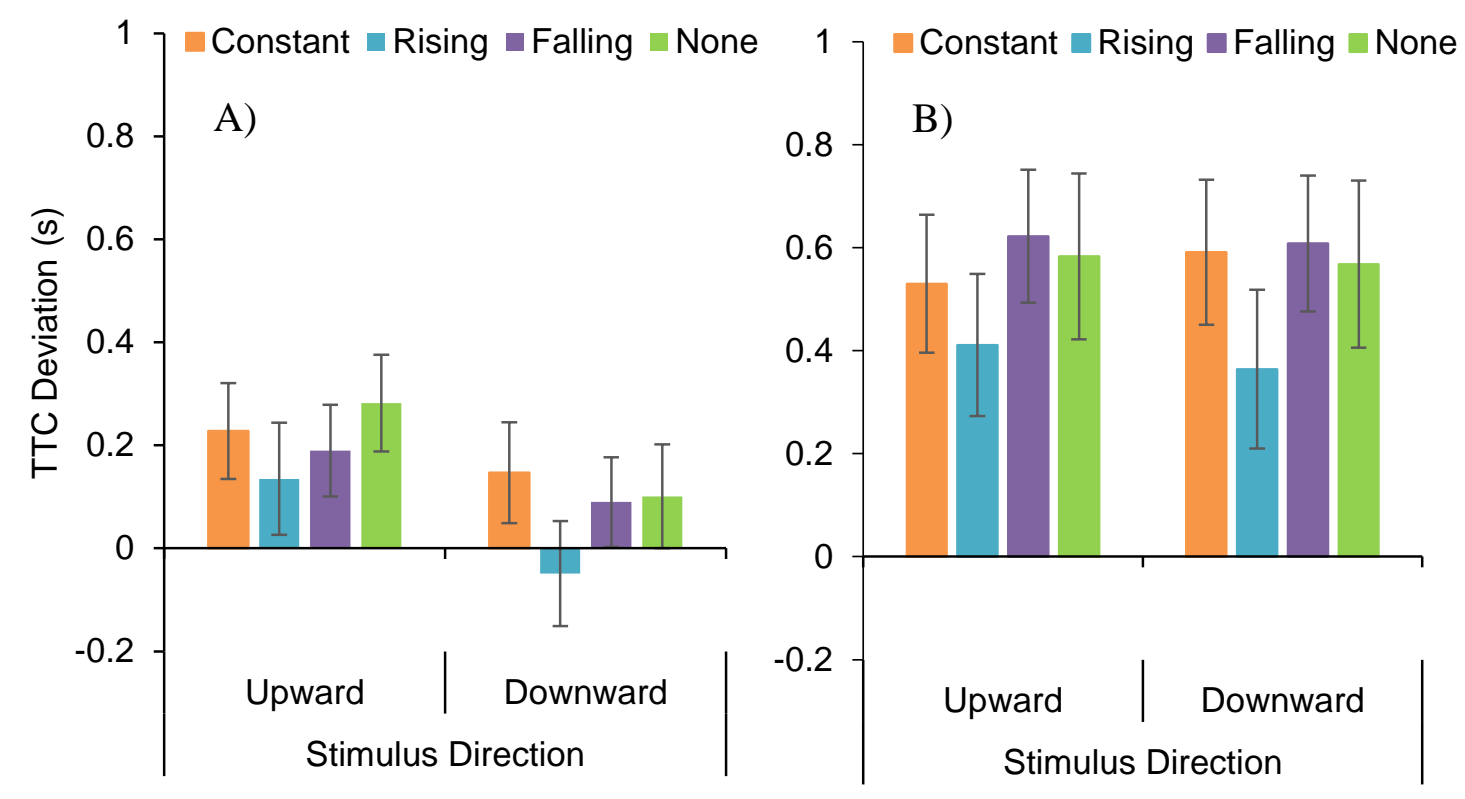

Figure 23. Mean TTC deviation for constant, rising, falling and silent trials in the no TMS condition for upward and downward stimuli for A) Experiment 1A (Values are for the slow stimulus speed and far landmark position only); and B) Experiment 2. Error bars reflect the standard error of the mean.

Experiment 2 produced greater overestimations when compared to Experiment 1A, as seen in Figure 23. The pattern of results in the upward condition seems to be consistent, with the exception of the falling pitch effect. In the downward condition, a rising pitch produced underestimations, which was not evident in Experiment 2. Another clear difference is the lack of gravity effect in Experiment 2; that is, TTC estimates are not lower for downward stimuli, as in Experiment 1A.

All TMS conditions. A 4 (TMS: left, right, vertex, no TMS) $\times 2$ (stimulus direction: upward, downward) $\times 4$ (pitch condition: constant, rising, falling, silent) repeated measures ANOVA was conducted to determine the effect of TMS on TTC deviations. 
There was no main effect of TMS, $F(3,33)=.48, p=.699, \eta p^{2}=.04$. There was a main effect of pitch, $F(3,33)=9.37, p<.001, \eta p^{2}=.46$, in which a rising pitch $(M=$ $0.42, S D=0.51)$ produced lower overestimations than a constant pitch $(M=0.54, S D=$ $0.49)$ and silent conditions $(M=0.60, S D=0.54)$; and a falling pitch produced higher overestimations $(M=0.63, S D=0.48)$, shown in Figure 24 .

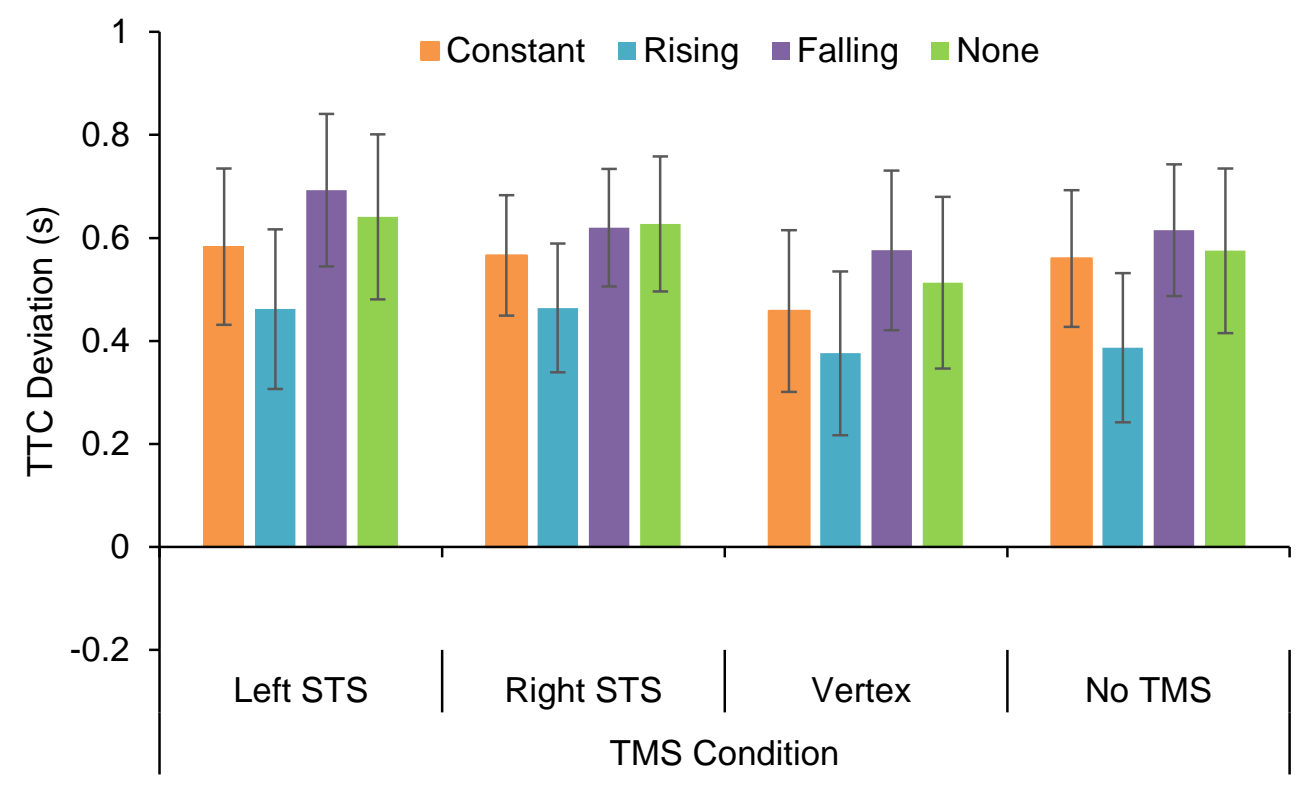

Figure 24. Deviations in TTC estimation for visual stimuli in the constant, rising, falling and silent pitch conditions by TMS condition. Error bars reflect the standard error.

The pitch effect mirrors the results of Experiment 1A, suggesting that, despite the increase in overestimations in the current experiment the pitch effects are still present.

\section{TTC Performance: Relative to Silent Baseline}

As TTC estimates were overestimations (slowed predictions overall; see Table $\mathrm{C} 1$ in the Appendix), TTC values were transformed to examine performance compared to the silent baseline, by subtracting the TTC deviation in the silent trials from deviation values for the remaining three pitch conditions (constant, rising, falling). Positive values indicate overestimations relative to the silent baseline condition (slower predicted motion) and negative values indicate underestimations relative to baseline (faster predicted motion). 
Baseline Performance. Again, TTC deviations were first examined for the no TMS condition only to gauge baseline performance. Figure $25 \mathrm{~B}$ ) shows a falling pitch produced overestimations in TTC, suggesting slowed predicted motion, and a rising pitch produced underestimations, suggesting speeded predicted motion. This reflects the pitch effects observed in Experiment 1A, and supports the hypothesis. Interestingly, a constant pitch produced overestimations for downward motion and underestimations for upward motion.

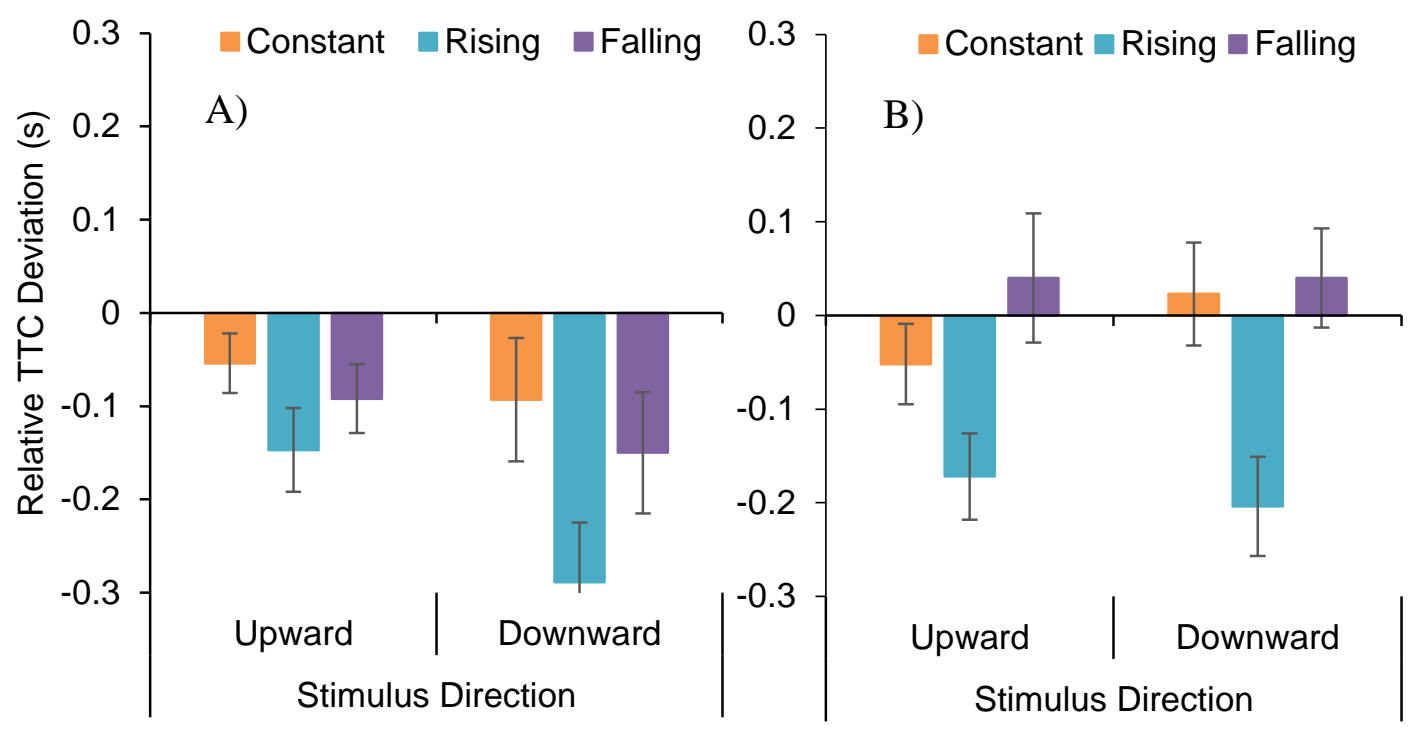

Figure 25. Relative TTC deviation for constant, rising, and falling and trials in the no TMS condition for upward and downward stimuli, relative to silent trials for A) Experiment 1A (Values are for the slow stimulus speed and far landmark position only); and B) Experiment 2. Error bars reflect the standard error of the mean.

When compared to the results of Experiment 2, Experiment 1A produced underestimations overall, however the difference between a rising and falling pitch remained.

\section{All TMS Conditions.}

A 4 (TMS: left, right, vertex, no TMS) $\times 2$ (stimulus direction: upward, downward) $\times 3$ (pitch condition: constant, rising, falling) repeated measures ANOVA was conducted to determine how TTC deviations for the rising and falling pitch conditions compared to those of the constant pitch condition.

There was no main effect of TMS, $F(3,33)=.45, p=.722, \eta p^{2}=.04$, or direction in this analysis, $F(1,11)=3.05, p=.109, \eta p^{2}=.28$. There was a main effect of pitch, $F(2,22)=18.02, p<.001, \eta p^{2}=.62$. Furthermore, there was a trending TMS $\times$ Pitch interaction, $F(6,66)=1.98, p=.081, \eta p^{2}=.15$, shown in Figure 26. Given the exploratory nature of this study, this predicted interaction was explored further. In all 
conditions, a rising pitch produced underestimations relative to a falling pitch. The interaction is driven by the fact that a rising pitch produced underestimations relative to a constant pitch in the no TMS and vertex control conditions, but not in either of the STS conditions. The interaction was further explored by examining effects in each TMS condition separately. One-way ANOVAs split by TMS condition showed a main effect of pitch for the left STS, $F(2,22)=14.32, p<.001, \eta p^{2}=.57$, right STS, $F(2,22)=$ $8.31, p=.002, \eta p^{2}=.43$, vertex, $F(2,22)=13.11, p<.001, \eta p^{2}=.54$, and no TMS conditions, $F(2,22)=16.34, p<.001, \eta p^{2}=.60$. However, follow-up t-tests showed that the differences between conditions lay in the comparison of a rising pitch to a constant pitch.

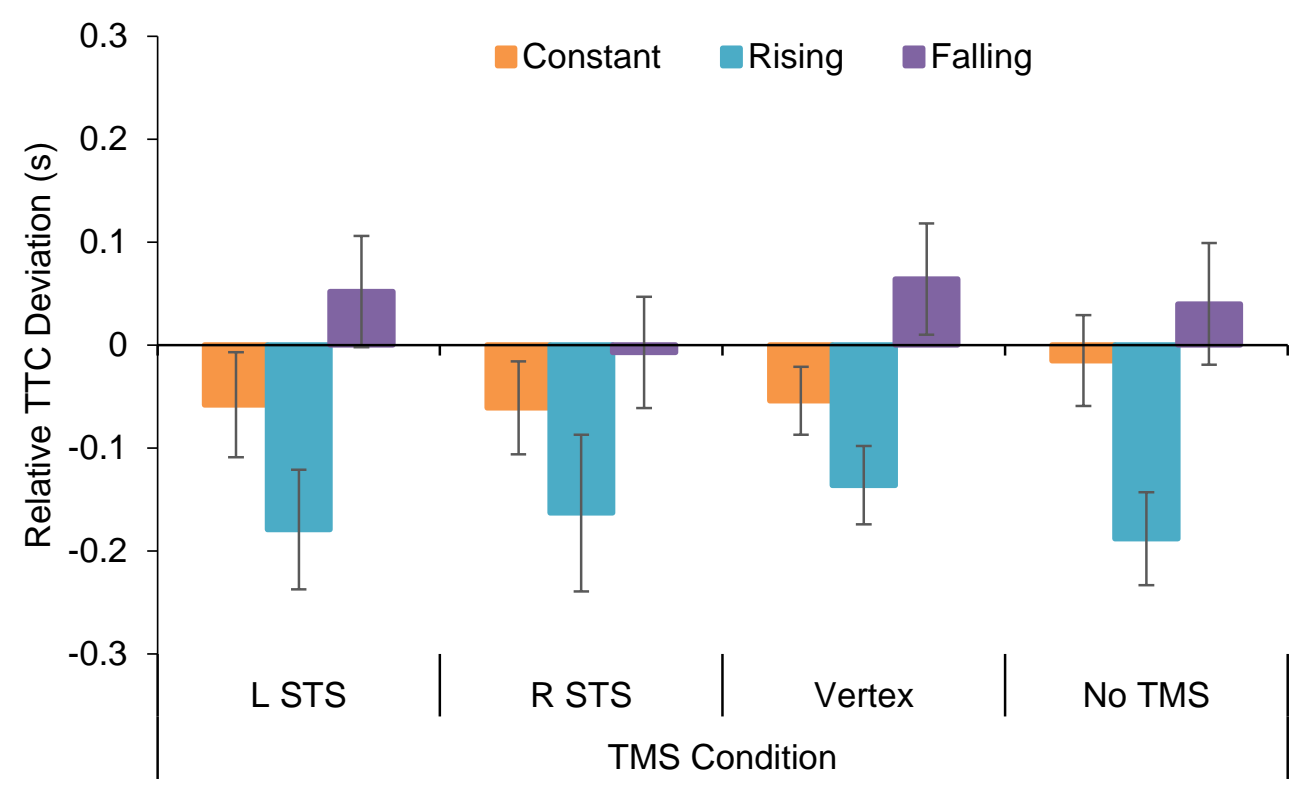

Figure 26. Relative deviations in TTC estimation for visual stimuli in the constant, rising and falling pitch conditions by TMS condition. Error bars reflect the standard error.

As shown in Figure 26, in the no TMS condition, a rising pitch produced underestimations compared to a falling, $t(11)=-4.43, p=.001, d=-1.28$, and a constant pitch, $t(11)=-4.87, p<.001, d=-1.40$, which did not differ from one another, $1.52, p=.156, d=0.44$. This result indicates an effect of pitch, in which predicted motion was sped up, driven by a rising pitch. When the vertex was stimulated, a rising pitch again produced underestimations compared to a falling, $t(11)=4.84, p=.001, d=$ -1.40 , and a constant pitch, $t(11)=-2.91, p=.014, d=-0.84$, which did not differ from each other, $t(11)=2.56, p=.027, d=0.74$. When the left STS was stimulated, a rising pitch produced significant underestimations compared to a falling pitch, $t(11)=-5.12, p$ $<.001, d=-1.48$, but not a constant pitch, $t(11)=-2.80, p=.017, d=-0.81$, which did 
not differ from each other, $t(11)=-2.68, p=.022, d=-0.77$, suggesting a general pitch effect. However this did not produce distortions in predicted motion as seen in the no TMS and vertex conditions. Similarly, when the right STS was stimulated, a rising pitch produced significant underestimations compared to a falling, $t(11)=-3.58, p=.004, d=$ -1.03, but not a constant pitch, $t(11)=-2.38, p=.037, d=-0.69$, which did not differ from each other, $t(11) 1.92, p=.081, d=0.55$, again suggesting only a general effect of pitch.

Summary. These results indicate a rising pitch effect in the no TMS condition and when stimulation was delivered to the vertex - the control site - evident by the significant difference between a rising and a constant pitch. This effect was attenuated when TMS was delivered to the left and right STS, indicating stimulation disrupted the speeding effect of the rising pitch. The specificity of TMS effects to the rising pitch is not surprising, given a rising pitch produced greater deviations in TTC compared to a falling pitch. A rising pitch was expected to exert greater effects than falling pitch in this experiment, due to the choice of the slow stimulus speed (a rising pitch exerted greater effects at the slow speed in Experiment 1A). The hypothesis was supported, as TMS to the left and right STS attenuated the effect of a rising pitch, but not a falling pitch. Therefore, it may be that a task using fast stimulus motion is needed in order to disrupt the effects of a falling pitch with TMS.

\section{General Discussion}

Visual motion judgements are essential in many everyday situations, such as gap-closure in traffic situations, and ball-catching in the sports field. Our perceptual systems are finely attuned to continue to make these judgements in occlusion situations (Battaglini et al., 2013; DeLucia et al., 2003, Peterken et al., 1991), such as when encountering an overtaking vehicle, or a blocking player. Judgements are most accurate when information is received from the visual and auditory modalities (Gordon \& Rosenblum, 2005; Hofbauer et al., 2004; Wuerger et al., 2010). However no TTC tasks have selectively manipulated the nature of an accompanying sound to determine how pitch and vision are integrated in predicted motion. This thesis examined the effect of pitch manipulations on predicted motion estimates; a valid question given the pitchspeed relationship (Collier \& Hubbard, 1998; Eitan \& Granot, 2004; McBeath \& Neuhoff, 2002).

Experiments $1 \mathrm{~A}$ and $1 \mathrm{~B}$ investigated whether visual motion imagery can be biased by manipulations in pitch, and under what circumstances the information would 
continue to be integrated. Experiment 2 examined whether the STS is the cortical locus responsible for binding the audio-visual stimuli responsible for the bias. In Experiment 1A, participants performed a horizontal and vertical TTC task with a changing pitch component. This pitch component consisted of a gradual rise or fall in pitch when the target reached the occlusion period. In both stimulus dimensions, a rising pitch speeded predicted motion, and a falling pitch slowed predicted motion relative to a constant pitch, consistent with the pitch-speed relationship (Collier \& Hubbard, 1998; Eitan \& Granot, 2004).

This bias was most pronounced at the far landmark position, which is consistent with previous literature suggesting memory of stimulus motion decay with increasing occlusion time (Gordon \& Rosenblum, 2005; Peterken et al., 1991). Given this explanation, a farther landmark position gives a longer period of time for pitch to create a distorted representation, and therefore a larger pitch bias.

\section{Interpreting the Behavioural Results}

The finding that pitch effects were affected by stimulus speed is supported by previous research that has also found a relationship between the two (Collier \& Hubbard, 1998; Eitan \& Granot, 2004; Eitan \& Tubul, 2010; McBeath \& Neuhoff, 2002; Neuhoff \& McBeath, 1996; Walker \& Ehrenstein, 2000). The relationship can be further explained by the modality appropriateness hypothesis (Welch \& Warren, 1980). This hypothesis stipulates different sensory modalities are suited to the perception of different events, and the information that is the most appropriate in a given situation is allocated the most attentional weighting. Therefore, during occlusion there is no visual input from the stimulus, only the memory of its velocity, thus the information provided by the dynamic pitch is given more weighting and affects predicted motion.

Dynamic pitch affected predicted motion when the stimulus speed was incongruent with the imagery induced by pitch. That is, slow speed and falling pitch are congruent - they both suggest a slowed speed - so there is no reason for pitch to affect predicted motion estimates. However, when the speed and pitch information were incongruent — for example slow speed and rising pitch—it makes more sense for the pitch information to be given more weighting, as the visual stimulus was occluded and the information about speed was unavailable.

In the vertical stimulus plane, the pitch effects were accompanied by a general gravity effect in the behavioural experiment, in which downward stimuli produced faster predicted motion. This finding supports the internal model view of gravity, which 
posits the laws of gravity are applied to downward movement, even when the situation does not require it (McIntyre et al., 2001; Zago et al., 2010; Zago \& Lacquaniti, 2005a; Zago \& Lacquaniti, 2005b; Zago et al., 2008). Importantly, the gravity effect was also observed in silent trials, indicating pitch is not necessary for the gravity effect to be observed. This result is consistent with that of Hubbard and Courtney (2009), who found larger forward displacement for descending visual stimuli than ascending (suggesting faster movement in the same period of time for downward moving stimuli), but they did not report whether their effect was altered by pitch.

In Experiment 1A, direction of stimulus movement interacted with pitch in the vertical plane. Downward stimuli were not always judged as moving faster than upward stimuli; fast-moving stimuli accompanied by a rising pitch were judged as faster in the upward condition. Therefore it is also possible spatial mappings-ascending pitch to high visual field and descending pitch to low visual field - interfered with the gravity effects in some circumstances, evidenced in some of the fast speed interactions (Takeshima \& Gyoba, 2013).

Factors Subserving Successful Integration. Experiment 1B examined whether a sudden change of pitch would still create effective integration of the audio-visual stimuli. No clear pitch biases were produced in this experiment. There are two possible explanations for this null result. Firstly, it could be due to the sudden nature of the pitch change; even though the pitch change was the same as the final pitch in Experiment 1A. The abrupt change in pitch could have disrupted the pairing of the sound with the visual stimulus, leading to separate processing. A second option is that it was not the sudden nature of the pitch change, but the fact the pitch did not continue to ascend or descend as in Experiment 1A. In other words, the visual stimulus was not predicted to be speeding or slowing in motion because the second pitch was not changing. These alternative explanations could easily be tested, and will be outlined later.

It is commonly viewed that two factors determine whether information from different modalities will be integrated and viewed as a unified event: spatial and temporal proximity of stimuli (Radeau, 1994; Schmiedchen, Freigang, Nitsche, \& Rübsamen, 2012). Additionally, the visual modality is thought to dominate spatial aspects of perception, whereas audition is dominant in temporal aspects of perception (Shams et al., 2002; Wada, Kitagawa, \& Noguchi, 2003; Watanabe \& Shimojo, 2001; Welch \& Warren, 1980). Spatial proximity of the sound source was not investigated in the current experiment, and therefore was held constant by equal presentation of sound 
to both ears. Temporal proximity was adhered to; pitch change always happened at the moment of visual occlusion. As such, it is unlikely the disruption to pitch-vision pairing was due to an issue with temporal correspondence.

Another, often unmentioned factor that determines separation or segregation of multimodal information is the degree of disparity between the two sources of information. If stimuli are similar enough, it is likely they will be interpreted as belonging to a single event and be co-bound. However, if stimuli are disparate, they are likely to be judged as two separate events and thus interpreted as originating from two separate sources (Körding et al., 2007; Schmiedchen et al., 2012). Indeed, the gradual change of pitch in Experiment 1A may have led to the judgement of a unified percept, likely due to the continuity of the sound. In Experiment 1B however, the pitch change was abrupt, jumping $100 \mathrm{~Hz}$ instantaneously. Therefore, the nature of this pitch change likely resulted in disruption of the speed illusion due to the sensory system perceiving the pitch change as two separate pitches when the constant pitch changed during occlusion.

The findings of Experiment 1A paved the way for an investigation into the cortical substrates of the pitch biases in Experiment 2. Experiment 2 investigated whether the left and right STS are responsible for binding the audio-visual components from Experiment 1A. The behavioural studies (1A and 1B) were constrained to only five repetitions of each experimental condition due to the number of variables explored. Experiment 2 allowed for 20 repetitions of each condition, as a reduced number of conditions were examined. Unexpectedly however, Experiment 2 yielded much higher overestimations than the same parameters when investigated in Experiment 1A. This may be explained by the difference in speed manipulations across experiments. It has been found that the speed of prior trials can influence estimates on following trials (Makin, Poliakoff, Chen, \& Stewart, 2008). Since Experiment 1A contained slow, medium and fast stimulus speeds, the presentation of a faster speed trial before a slow speed trial due to random assignment could have meant participants interpreted slowspeed trials as moving faster than they would have had speed been blocked instead of randomised in experiments. In Experiment 2, only one stimulus speed was presented, which did not allow for previous speeds to impact on predicted motion, perhaps slowing the predictions comparatively.

Another explanation is that changing the nature of stimulus onset in Experiment 2 - automatic initiation of movement compared to user-controlled movement in 
Experiment 1A - interfered with stimulus speed estimations as participants were not prepared. Experiment 1A inadvertently gave participants extra time to prepare to make distance judgements. Therefore, when the stimulus started, they were better able to match the extrapolated speed information with the stored distance information and give the correct TTC duration. Experiment 2 did not provide the possibility of an extra window of time for participants to calibrate TTC judgements. Therefore, the results obtained in this experiment may form a more indicative view of behaviour in situations involving object occlusion.

Another difference between Experiment 1A and Experiment 2 was there was no gravity effect observed in the silent trials in Experiment 2. This result conflicts with other studies which have investigated constant motion in the vertical plane without the addition of sound (Hubbard, 1997; Nagai, Kazai, \& Yagi, 2002; Senot, Zago, Lacquaniti, \& McIntyre, 2005). These studies have found faster predicted motion for downward compared to upward motion, consistent with the results of $1 \mathrm{~A}$ and $1 \mathrm{~B}$ (Hubbard, 1997; Nagai et al., 2002; Senotet al., 2005). The lack of gravity effect observed in Experiment 2 has not been observed in vertical TTC studies previously. Although different from the vertical motion effects observed in Experiments 1A and $1 \mathrm{~B}$, it must be recognised that there was no actual gravity force acting, or suggested in the current experiments. This result suggests that under the parameters used in Experiment 2, downward motion was not predicted as moving faster than upward motion.

\section{Interpreting the TMS Results}

In addition to the behavioural differences, TMS stimulation to the right and left STS did not produce a main effect of TMS. However, this was to be expected, as a main effect of TMS would have meant a disruption of general motion prediction. It was hypothesised that inhibitory TMS over the left and right STS would produce a disruption in the pitch bias, causing a rising and a falling pitch to produce the same TTC estimates as a constant pitch. This prediction was based on previous research which has found the STS to be implicated in the binding of audio-visual stimuli in multisensory tasks (Barraclough, Xiao, Baker, Oram, \& Perrett, 2005; Beauchamp et al., 2004; Hocking \& Price, 2008; Noesselt, Bergmann, Heinze, Münte, \& Spence., 2012; Marchant, Ruff, \& Driver, 2012; Noesselt et al., 2007; Stevenson \& James, 2009; Stevenson, Mullin, Wallace, \& Steeves, 2013; Werner \& Noppeney, 2009). The TMS $\times$ Pitch interaction was trending, indicating a slight disruption to the rising pitch effect at 
both the left and right STS sites. There are several reasons that could have affected the strength of this interaction.

Low participant numbers (due to time and resource constraints) could have been an issue in Experiment 2. Specifically, low participant numbers may have resulted in an underpowered study, meaning it was not possible to obtain the significant TMS $\times$ Pitch interaction, even if it was there (VanVoorhis \& Morgan, 2007). There was a pattern of results suggesting slight inhibition of rising pitch effects after stimulation of the left and right STS. Although small participant numbers are standard in studies using cTBS, with many using numbers of twelve or less (Bertini et al., 2010; van Kemenade, Muggleton, Walsh, \& Saygin, 2012; Rahnev et al., 2013; Tarnutzer et al., 2013), it still could have been an issue in the current study. Even so, the results are likely explained better by three other factors: the method used to localise the stimulation site, the orientation of the TMS coil itself, and the method used to determine the motor threshold. Optimising these factors should increase power to detect TMS effects in future studies.

Localisation of Stimulation Site. Evidence supporting the number of participants in the current study is indicated in Beauchamp et al. (2010), who investigated whether the STS is the cortical region responsible for the McGurk effect, in which auditory speech sounds are altered by incongruent visual speech (McGurk \& MacDonald, 1976). Beauchamp and colleagues had participants engage in a multisensory task consisting of McGurk, or non-McGurk stimuli, while simultaneously receiving TMS to the STS. Importantly, TMS created a significant decline in the percentage of participants that experienced the McGurk effect. Beauchamp and colleagues obtained this significant result using 12 participants. This information suggests the number of participants used in the current study did not confound the results. A difference between the current study and that of Beauchamp and colleagues lies in the method used to localise the multisensory area of the STS, and could explain the discrepancy in results.

In TMS studies, target areas can be located via five methods: (a) localisation using the 10/20 EEG system, (b) a function-guided approach, (c) localisation using Talairach coordinates derived from previous studies, (d) individual MRI data using anatomical landmarks, or (e) fMRI-guided frameless stereotaxic localisation (Sparing, Buelte, Meister, Pauš, \& Fink, 2008).

The 10/20 system relies on landmarks (the preauricular points, the nasion and the inion) and the space between them to locate target sites. It can be very precise due to 
the mathematical nature of localisation. However, this method assumes that all participants have the same head structure, and that the brain sites lie directly below the outlined coordinates (Sparing et al., 2008). It is estimated that using the 10/20 method would fail to localise the correct site in $10 \%$ of participants (Herwig, Satrapi, Schönfeldt-Lecuona, 2003).

Instead, it is better to use a function-guided approach, in which the coil location is guided by the effect elicited by stimulation to that area (Sandrini et al., 2011). For example, stimulation of the hand area in the primary motor cortex elicits visible twitches in the fingers of the hand (Julkunen et al., 2009). However, this approach is not useful for behaviourally silent areas—-such as the multisensory region in the STSwhich do not elicit visible behavioural effects (Julkunen et al., 2009).

One way to target behaviourally silent cortical regions with TMS is by using Talairach coordinates from other studies that have targeted the same region. Talairach coordinates are usually obtained by taking an average of coordinates from multiple studies targeting the area of interest (Sparing et al., 2008). A problem with this approach is in the use of studies; they have to be similar enough as to recruit the same cortical regions. In addition, using Talairach coordinates does not take into account differences in brain anatomy between participants (Sparing et al., 2008). Specifically, the coordinates for one brain region might not coincide with the same exact region between participants.

Using individual MRI scans amends this issue, as it takes into account individual variation in skull size and layout of brain structures (Sack et al., 2009). However, like the aforementioned methods, this method also has its disadvantages. This approach is only useful if the area of interest is easily located visually. If the region to be localised does not have an identifiable landmark, this method is not useful. For example, the hand area in the primary motor cortex can be identified by a structure resembling an inverted omega, which protrudes into the central sulcus (Sparing et al., 2009). However, the multisensory region in the posterior STS does not have a visually identifiable landmark.

The best method for localising a region for stimulation with TMS is the fMRIguided approach. This approach identifies the area of interest by acquiring task-related BOLD activations, controlling for individual differences in anatomy and function (Sparing, 2008). Indeed, a study which targeted the prefrontal cortex (a behaviourally silent area) found significant effects when the TMS coil was positioned via the fMRI- 
guided technique, but not when using coordinates from group averages (Feredoes, Tononi, \& Postle, 2007).

Sack et al. (2009) investigated four of these methods: fMRI-guided frameless stereotaxic localisation, individual MRI data using anatomical landmarks, localisation using Talairach coordinates derived from previous studies, and localisation using the 10/20 EEG system. They did not investigate the efficacy of a function-guided approach. The largest effect size was found using an fMRI-guided method, followed by MRIguided, localisation by Talairach coordinates, then by and anatomical structures. This is consistent with Sparing and colleagues (2008). However, Sack and colleagues (2009) went beyond a simple efficacy analysis by determining the number of participants needed to find an effect with each method. Sack and colleagues found a significant effect for fMRI-guided localisation in their study by using only five participants. They concluded that in order to find significant effects by using the remaining methods, a number of nine participants would be needed if using the individual MRI-guided procedure, 13 for the Talairach coordinates method, and 47 using the 10/20 system.

The current study used a combined method of Talairach coordinates and localisation via individual MRI scans. This method was chosen as it provided much better localisation than by using the 10/20 system or by using individual MRI scans or Talairach coordinates alone. Talairach coordinates obtained in a meta-analysis by Hein and Knight (2008) were transformed into MNI coordinates. These MNI coordinates were used to locate the multisensory area broadly for each individual, then individual MRI scans were used to ensure these coordinates lay on the sulcus of the STS. If necessary, the target point was moved to coincide with the sulcus. This combined approach took into account individual variation between individuals by use of MRI scans, while avoiding the potential problem of locating the wrong visual landmarks by using the Talairach coordinates. This method was the best approach in the current experiment, given the resources available. However, an even better approach would have been to use an fMRI-guided approach. An fMRI-guided approach would have meant the area in the STS that was targeted would be one which shows the most activation for multisensory stimuli (Calvert, 2001).

Another advantage to the fMRI-guided approach in localising the STS is realised when the spatial organisation of the STS itself is considered. It is thought that areas in the STS which respond to multisensory stimuli are clustered together in close spatial proximity (van Atteveldt, Blau, Blomert, \& Goebel, 2010; Beauchamp et al., 
2004; Calvert et al., 2001; Dahl, Logothetis, \& Kayser, 2009). However, the prevalence of neurons that respond to multisensory information is smaller than those with unimodal specific responses (Beauchamp et al., 2004; Hikosaka, Iwai, Saito, \& Tanaka, 1988). In a study determining the architecture of the STS, Beauchamp et al. (2004) found only $26 \%$ of neurons in the multisensory region of the STS responded to both auditory and visual stimuli equally, whereas $30 \%$ preferred visual, and $44 \%$ preferred auditory stimuli. Similarly, Hikosaka et al. (1988) found only $10.5 \%$ of neurons in the posterior STS of monkeys to be multisensory. These values allow for a very small spatial window when trying to target the multisensory region in the STS. Therefore, it is clear that the neurons in the STS are heterogeneous in function, which suggests the TMS stimulation may not have encompassed multisensory neurons in this study. A functional imaging approach would have shown BOLD enhancement in this multisensory region of the STS, ensuring the coil was positioned in the correct location for each individual (Sparing et al., 2008)

Coil Orientation. Another factor that may have impacted the results is the orientation of the coil. It is known that coil orientation has differential effects on the strength of the electric field and the depth at which the current reaches, therefore influencing the degree of inhibition at the neuronal level (Laakso, Hirata, \& Ugawa, 2014). Coil orientation could therefore pose a problem with consistency in level of disruption if the orientation is not fixed. Indeed, the influence of changes in coil orientation has been systematically investigated by Thomson et al. (2013), using TMS and near infra-red spectroscopy to assess changes in blood oxygenation $(\mathrm{HbO})$ in the left prefrontal cortex. Thomson et al. used both single pulse and rTMS to determine the best coil orientation of three orientations: $45^{\circ}, 135^{\circ}$, and $225^{\circ}$ in a counter-clockwise direction from the midline. For single pulse TMS, a reduction in $\mathrm{HbO}$ was observed when coil orientation was at $45^{\circ}$ which was not evident at the other two orientations. As a decrease in $\mathrm{HbO}$ levels is a marker of neural inhibition (Stefanovic, Warnking, \& Pike, 2014), this determines $45^{\circ}$ as the optimum angle to cause inhibition of the prefrontal cortex.

Similarly, studies targeting the motor cortex with TMS also commonly orient the coil so the handle is pointing back towards the occiput, at a $45^{\circ}$ angle to the midsagittal plane (Gangitano, Mottaghy, \& Pascual-Leone, 2004; Gentner et al., 2008; Goldsworthy et al., 2013; Tsang et al., 2014). However, optimal coil orientation has not been investigated in the STS. Prior studies targeting this region have used varying 
orientations: coil handle pointing backwards and parallel to the mid-sagittal plane (Dzhelyova, Ellison, \& Atkinson, 2011), handle pointing backwards at a $45^{\circ}$ angle (Candidi, Stienen, Aglioti, \& de Gelder, 2011), and handle pointing upwards (Tarnutzer et al., 2013). However, it is not uncommon for studies targeting the STS to omit coil orientation details (Beauchamp et al., 2010; Grossman et al., 2005; van Kemenade et al., 2012), posing a problem with consistency across studies.

In the current experiment, the coil was oriented at approximately $45^{\circ}$ from the mid-sagittal plane. However, a different orientation was sometimes necessary due to the close proximity of the ear to the target location, so that the wings of the coil did not encroach on the ear and cause physical discomfort. In this instance, the coil handle was always posterior facing and within the range of $0-90^{\circ}$. Furthermore, the STS was always verified by using the neuronavigator system. A changing coil orientation has been utilised in another study using TMS on the STS. Cattaneo et al. (2010) reported a vertical coil orientation with an upward pointing handle, but reported changing the coil orientation when the ear became obstructed to minimise physical discomfort. The support of a variable coil orientation in the current study by Cattaneo and colleagues, suggests at present it may be the best solution to targeting a cortical site which is sometimes obstructed. However it is clear that an optimum coil orientation needs to be determined for TMS studies targeting the STS, as there is no current protocol for experimenters to adhere to.

Motor Threshold Determination. Finally, two participants dropped out of Experiment 2 due to finding the stimulation intensity too severe; this attrition decreased the power of the study. Dropout may have been avoided by changing the method used to find the motor threshold. Experiment 2 used observed muscle movements to determine the motor threshold (Pridmore, as cited in Westin et al., 2014). However, some researchers believe this technique to be inferior to other methods (Julkunen et al., 2008; Tranulis et al., 2006; Westin et al., 2014). In particular, Westin et al. (2014) considers the observation method to cause overestimations in motor threshold estimates, and by extension, use of a stimulation intensity that is too high. A more accurate method of detecting the motor threshold is by using electromyography (EMG) to find the lowest intensity level at which a motor evoked potential (MEP) of $50 \mu \mathrm{V}$ occurs in five out of 10 consecutive trials (Westin et al., 2014). Westin et al. obtained motor thresholds of 20 participants using both MEPs and observable muscle twitches, and found that the latter technique caused overestimations in motor thresholds by an 
average of $11.3 \%$. If this overestimation percentage is applied to the current study, instead of being stimulated at 51\%, participants five and six (who dropped out of the current study) would have received stimulation at an intensity of $46 \%$. This intensity is more likely to have been tolerated.

To summarise, the results obtained in Experiment 1A were consistent with the pitch-speed relationship (Collier \& Hubbard, 1998; Eitan \& Granot, 2004; McBeath \& Neuhoff, 2002), and evidence for memory decay with increasing occlusion time (Gordon \& Rosenblum, 2005; Peterken et al., 1991). Experiment 1B did not produce results consistent with $1 \mathrm{~A}$, which could be explained by factors which determine integration, such as the disparity between the pitch stimuli (Körding et al., 2007; Schmiedchen et al., 2012). The results of Experiment 1B are also consistent with the modality appropriateness hypothesis (Welch \& Warren, 1980). Specifically, it could be that the pitch information was assigned more weighting during integration as the visual information was is no longer available. Nevertheless, both Experiments 1A and 1B produced gravity effects in the vertical plane, consistent with the internal model view of gravity ((McIntyre et al., 2001; Zago et al., 2010; Zago \& Lacquaniti, 2005a; Zago \& Lacquaniti, 2005b; Zago et al., 2008).

The behavioural results of Experiment 2 could be explained by lack of carryover speed effects which could have been present in Experiment 1A (Makin et al., 2008). Furthermore, Experiment 2 suggested that the combination of parameters used do not produce a gravity effect in small sample sizes. Four methodological considerations were discussed in terms of the TMS results observed in Experiment 2. Low participant numbers could have been an issue, despite the fact that small sample sizes are common in the cTBS literature ((Bertini et al., 2010; van Kemenade, Muggleton, Walsh, \& Saygin, 2012; Rahnev et al., 2013; Tarnutzer et al., 2013). However, this was to be expected given the pilot nature of the current study. The localisation method used in the current study was superior to methods such as the 10/20 method, and localisation via individual MRI scans or Talairach coordinates alone. However, a better approach would have been to use fMRI-guided method, as this allows areas of multisensory integration to guide localisation (Sparing et al., 2008). Another consideration was the coil orientation. Coil orientation was consistent in current experiment, apart from instances in which the ear was touching the wings of the coil. Support for this method is obtained from a study using a changing coil orientation (Cattaneo et al., 2010). Studies have suggested that changing the orientation of the coil 
can change the effects of TMS at the neuronal level (Laakso, Hirata, \& Ugawa, 2014). However, although optimum coil orientation has been investigated areas such as the primary motor cortex (Thomson et al., 2013), such an investigation is yet to happen with the STS. Finally, the method used to determine participant motor thresholds could have been improved by the use of MEPs (Westin et al., 2014).

\section{Future Directions}

Having now discussed the results of the current studies and determined factors which could have contributed to the results, I will now present avenues that should be investigated in future research. In regards to the behavioural manipulations of the current study, it would be important to determine whether the lack of pitch effect in Experiment 1B was due to the disruption of the bias by the sudden change in pitch, or whether it was the loss of the dynamic nature of the pitch. A way to investigate this would be to replicate and improve upon the main procedure of Experiment 1B. Instead of the pitch changing from $500 \mathrm{~Hz}$ to $400 \mathrm{~Hz}$ or $600 \mathrm{~Hz}$ and then staying constant at the point of occlusion, a gradual pitch change after the sudden jump would need to occur at $18 \mathrm{~Hz} / \mathrm{s}$, as in Experiment 1A. If this version does not produce a pitch bias, it is likely the disruptive nature of missing out $100 \mathrm{~Hz}$ supressed bimodal integration. However, if a pitch effect is evident, it is likely that the fact that pitch can cause biases in speed is due to the accelerating/decelerating nature, which is metaphorically construed as speeding/slowing (Collier \& Hubbard, 1998).

In Experiment 1A, it was determined that rising pitch effects are greatest at slow stimulus speeds, and falling pitch effects at fast stimulus speeds. Experiment 2 investigated only one of these speeds; the slow speed, and found inhibition in the left and right STS for a rising pitch only. An important follow up study would be to examine whether TMS to the left and right STS could produce inhibition of the falling pitch effect in a task with fast stimulus motion. If this result is observed, then this would implicate the STS in the integration of dynamic pitch with visual information in TTC tasks. Furthermore, if pitch and speed form a delicate push-pull relationship in the way the present results have suggested, it should be possible to alter the magnitude, and even the presence of pitch effects by changing the stimulus speeds.

Additionally, due to time constraints, Experiment 2 only investigated whether TMS to the STS disrupts auditory integration with vertically moving stimuli. Since vertical motion encompasses gravity effects, it would also be important to investigate 
TTC in a paradigm with horizontal motion only, allowing for a thorough investigation of pitch effects.

An important aspect to incorporate into the design of this new TMS study would be the utilisation of the fMRI-guided localisation method, which takes into account individual differences in structure-function relationships (Sparing et al., 2008). FMRI activations would be expected in regions involved in processing the visual, auditory and audio-visual aspects of the task, as well as activation in motor areas and regions involved in cognition (Beauchamp, 2005). Therefore, it would be important to include TTC trials in which estimates are based on only the sound stimuli, as well as the visualonly baseline condition. Activation can be computed by using an approach which expects superadditivity for multisensory regions, or by expecting a larger mean response to multisensory stimuli than unimodal stimuli (Calvert, 2001). By either method, subtracting cortical regions which respond only to unimodal stimuli, and regions while respond to cognitive and motor responses should leave multisensory regions of activation. Additionally, some cortical areas are active at rest (Beauchamp, 2005). As such, it would be important to take a baseline measure of activity for each participant for ten minutes before starting the trial to determine areas active at rest.

In the current TMS study, the vertex was used as a control-site for the nonspecific effects of TMS (such as the clicking of the coil and physical sensation of the stimulation). This type of control is called off-target active stimulation; participants still receive active TMS stimulation, but at a site which does not cause behavioural effects. The utilisation of off-target active stimulation did not cause issues in the current study. However, a better control would have been the use of a sham coil, which is applied to the same site as the active target stimulation. Sham coils do not exert physical effects, but are (if the sham is successful) indistinguishable from active stimulation at the target-site. Advanced sham coils have a magnetic shield, ensuring no neural inhibition occurs (Duecker \& Sack, 2015). However, this approach lacks the somatosensory effects of an active coil, and can be immediately distinguished from an active coil, particularly when using experienced TMS participants. In order to counteract this disadvantage, it is possible to use time-locked surface electrodes to stimulate the surface of the skin, creating the somatosensory effects, without affecting behaviour (Duecker \& Sack, 2015). This combination seems a promising compromise to vertex stimulation, especially when using participants familiar with TMS who may notice a somatosensory difference. 


\section{Conclusions}

This thesis examined the influence of pitch and direction on visual motion judgements in a TTC task. Since dynamic pitch was a novel addition to a TTC paradigm, Experiments 1A and 1B also examined other parameters which might affect TTC estimates: landmark position and stimulus speed. It was hypothesised that a rising pitch would elicit underestimations due to speeded predicted motion and that a falling pitch would elicit overestimations due to slowed predicted motion. Additionally, in the vertical dimension, gravity effects were postulated in which downward motion was expected to speed predicted motion compared to upward motion. Experiment 1A featured a gradual pitch change at the stimulus occlusion period. In this experiment, the predictions were supported in relation to a constant pitch, as the mere presence of a sound altered TTC estimations compared to silent trials. In Experiment 1B, the pitch change was sudden, and did not continue to change in pitch with increasing occlusion. Pitch effects were not consistently observed in Experiment 1B, which can possibly be attributed to the sudden nature of the change, resulting in a disruption of the audiovisual binding. Gravity effects were observed in both experiments even under pitch conditions, suggesting the rising-falling pitch difference was consistent for both upward and downward motion, and suggesting that pitch and gravity produce additive effects on predicted motion.

Experiment 2 was conducted to determine if the STS is responsible for the audio-visual interactions observed in Experiment 1A. It was hypothesised that TMS would inhibit the pitch-effects, such that a rising and falling pitch produced TTC estimates similar to a constant pitch, while leaving the gravity effect intact. TMS to the STS did not significantly reduce TTC estimates for falling pitch trials, although this was expected due to the speed of stimuli in Experiment 2. Therefore the first part of the hypothesis was supported. However, follow up studies are needed to determine if the STS is also involved in integrating visual motion with a falling pitch in TTC paradigms.

The different effects observed in Experiments 1A and 1B are informative about stimulus factors necessary for successful multimodal integration, contributing to existing literature on the matter (e.g. Körding et al., 2007; Schmiedchen et al., 2012; Welch \& Warren, 1980). More importantly however, these novel experiments act as a link between the literature on pitch-speed relationships (Collier \& Hubbard, 1998; Eitan \& Granot, 2004; McBeath \& Neuhoff, 2002) and the influence of sound on real or imagined visual motion (Maeda et al., 2004; Manabe \& Riquimaroux, 2000; Shams et 
al., 2002; Watanabe \& Shimojo, 2001). This thesis has determined that dynamic pitch change can indeed influence speed estimates in TTC tasks, and that the STS seems to be involved in the integration of the audio-visual components. Findings from these experiments suggest that the TTC paradigm may be useful in capturing audio-visual integration in future studies of predicted motion. 


\section{References}

Allison, T., Puce, A., \& McCarthy, G. (2000). Social perception from visual cues: role of the STS region. Trends in cognitive sciences, 4(7), 267-278. doi: 10.1016/S1364-6613(00)01501-1

Audacity (Version 2.0.5) [software]. (2008). Retrieved from http://web.audacityteam.org/

Barraclough, N. E., Xiao, D., Baker, C. I., Oram, M. W., \& Perrett, D. I. (2005). Integration of visual and auditory information by superior temporal sulcus neurons responsive to the sight of actions. Journal of Cognitive Neuroscience, 17(3), 377-391. doi: 10.1162/0898929053279586

Battaglia, P. W., Jacobs, R. A., \& Aslin, R. N. (2003). Bayesian integration of visual and auditory signals for spatial localization. JOSA A, 20(7), 1391-1397. doi: 10.1364/JOSAA.20.001391

Battaglini, L., Campana, G., \& Casco, C. (2013). Illusory speed is retained in memory during invisible motion. $i$-Perception, 4(3), 180-191. doi: 10.1068/i0562

Bear, M. F., Connors, B. W., \& Paradiso, M. A. (Eds.). (2007). Neuroscience (Vol. 2). Lippincott Williams \& Wilkins.

Bertini, C., Leo, F., Avenanti, A., \& Ladavas, E. (2010). Independent mechanisms for ventriloquism and multisensory integration as revealed by theta-burst stimulation. European Journal of Neuroscience, 31(10), 1791-1799. doi: 10.1111/j.1460-9568.2010.07200.x

Beauchamp, M. S. (2005). See me, hear me, touch me: multisensory integration in lateral occipital-temporal cortex. Current opinion in neurobiology, 15(2), 145153. doi: 10.1016/j.conb.2005.03.011

Beauchamp, M. S., Lee, K. E., Argall, B. D., \& Martin, A. (2004). Integration of auditory and visual information about objects in superior temporal sulcus. Neuron, 41(5), 809-823. doi: 10.1016/S0896-6273(04)00070-4

Beauchamp, M. S., Nath, A. R., \& Pasalar, S. (2010). fMRI-guided transcranial magnetic stimulation reveals that the superior temporal sulcus is a cortical locus of the McGurk effect. The Journal of Neuroscience, 30(7), 2414-2417. doi: 10.1523/JNEUROSCI.4865-09.2010

Bischoff, M., Walter, B., Blecker, C. R., Morgen, K., Vaitl, D., \& Sammer, G. (2007). Utilizing the ventriloquism-effect to investigate audio-visual binding. 
Neuropsychologia, 45(3), 578-586. doi:

10.1016/j.neuropsychologia.2006.03.008

Bootsma, R. J., \& Oudejans, R. R. (1993). Visual information about time-to-collision between two objects. Journal of Experimental Psychology: Human Perception and Performance, 19(5), 1041- 1052. doi: 10.1037/0096-1523.19.5.1041

Calabro, F. J., Beardsley, S. A., \& Vaina, L. M. (2011). Different motion cues are used to estimate time-to-arrival for frontoparallel and looming trajectories. Vision research, 51(23), 2378-2385. doi: 10.1016/j.visres.2011.09.016

Calvert, G. A. (2001). Crossmodal processing in the human brain: insights from functional neuroimaging studies. Cerebral cortex, 11(12), 1110-1123. doi: 10.1093/cercor/11.12.1110

Calvert, G. A., Campbell, R., \& Brammer, M. J. (2000). Evidence from functional magnetic resonance imaging of crossmodal binding in the human heteromodal cortex. Current biology, 10(11), 649-657. doi: 10.1016/S0960-9822(00)00513-3

Calvert, G. A., Hansen, P. C., Iversen, S. D., \& Brammer, M. J. (2001). Detection of audio-visual integration sites in humans by application of electrophysiological criteria to the BOLD effect. Neuroimage, 14(2), 427-438. doi:

10.1006/nimg.2001.0812

Candidi, M., Stienen, B. M., Aglioti, S. M., \& de Gelder, B. (2011). Event-related repetitive transcranial magnetic stimulation of posterior superior temporal sulcus improves the detection of threatening postural changes in human bodies. The Journal of Neuroscience, 31(48), 17547-17554. doi:

10.1523/JNEUROSCI.0697-11.2011

Cattaneo, L., Sandrini, M., \& Schwarzbach, J. (2010). State-dependent TMS reveals a hierarchical representation of observed acts in the temporal, parietal, and premotor cortices. Cerebral Cortex, 20(9), 2252-2258. doi:

10.1093/cercor/bhp291

Chikazoe, J., Konishi, S., Asari, T., Jimura, K., \& Miyashita, Y. (2007). Activation of right inferior frontal gyrus during response inhibition across response modalities. Journal of Cognitive Neuroscience, 19(1), 69-80. doi: 10.1162/jocn.2007.19.1.69

Chiou, R., \& Rich, A. N. (2012). Cross-modality correspondence between pitch and spatial location modulates attentional orienting. Perception, 41(3), 339-353. doi: $10.1068 / \mathrm{p} 7161$ 
Collier, W. G., \& Hubbard, T. L. (1998). Judgments of happiness, brightness, speed and tempo change of auditory stimuli varying in pitch and tempo. Psychomusicology: Music, Mind \& Brain, 17(1), 36-55. doi: 10.1037/h0094060

Coull, J. T., Vidal, F., Goulon, C., Nazarian, B., \& Craig, C. (2008). Using time-tocontact information to assess potential collision modulates both visual and temporal prediction networks. Frontiers in human neuroscience, 2, 1-12. doi: 10.3389/neuro.09.010.2008

Dahl, C. D., Logothetis, N. K., \& Kayser, C. (2009). Spatial organization of multisensory responses in temporal association cortex. The Journal of Neuroscience, 29(38), 11924-11932. doi: 10.1523/JNEUROSCI.3437-09.2009

DeLucia, P., Kaiser, M., Bush, J., Meyer, L., \& Sweet, B. (2003). Information integration in judgements of time to contact. The Quarterly Journal of Experimental Psychology, 56A(7), 1165-1189. doi: $10.1080 / 02724980343000053$

DeLucia, P. R., \& Liddell, G. W. (1998). Cognitive motion extrapolation and cognitive clocking in prediction motion tasks. Journal of Experimental Psychology: Human Perception and Performance, 24(3), 901-914. doi: 10.1037/00961523.24.3.901

Duecker, F., \& Sack, A. T. (2015). Rethinking the role of sham TMS. Frontiers in psychology, 6, 210-214. doi:10.3389/fpsyg.2015.00210

Dzhelyova, M. P., Ellison, A., \& Atkinson, A. P. (2011). Event-related repetitive TMS reveals distinct, critical roles for right OFA and bilateral posterior STS in judging the sex and trustworthiness of faces. Journal of cognitive neuroscience, 23(10), 2782-2796. doi: 10.1162/jocn.2011.21604

Eitan, Z., \& Granot, R. Y. (2004). Musical parameters and images of motion. In Conference on Interdisciplinary Musicology (CIM04), Graz, Austria.

Eitan, Z., \& Tubul, N. (2010). Musical parameters and children's images of motion. Musicae Scientiae, 14(2 suppl), 89-111.

Feredoes, E., Tononi, G., \& Postle, B. R. (2007). The neural bases of the short-term storage of verbal information are anatomically variable across individuals. The Journal of Neuroscience, 27(41), 11003-11008. doi: 10.1523/JNEUROSCI.1573-07.2007

Formisano, E., Linden, D. E., Di Salle, F., Trojano, L., Esposito, F., Sack, A. T., ... \& Goebel, R. (2002). Tracking the mind's image in the brain I: time-resolved fMRI 
during visuospatial mental imagery. Neuron, 35(1), 185-194. doi: 10.1016/S0896-6273(02)00747-X

Freeman, E., \& Driver, J. (2008). Direction of visual apparent motion driven solely by timing of a static sound. Current biology, 18(16), 1262-1266. doi :

10.1016/j.cub.2008.07.066

Gangitano, M., Mottaghy, F. M., \& Pascual-Leone, A. (2004). Modulation of premotor mirror neuron activity during observation of unpredictable grasping movements. European Journal of Neuroscience, 20(8), 2193-2202. doi: 10.1111/j.14609568.2004.03655.x

Gentner, R., Wankerl, K., Reinsberger, C., Zeller, D., \& Classen, J. (2008). Depression of human corticospinal excitability induced by magnetic theta-burst stimulation: evidence of rapid polarity-reversing metaplasticity. Cerebral cortex, 18(9), 2046-2053. doi: 10.1093/cercor/bhm239

Ghazanfar, A. A., \& Schroeder, C. E. (2006). Is neocortex essentially multisensory? Trends in cognitive sciences, 10(6), 278-285. doi: 10.1016/j.tics.2006.04.008

Godfrey, H. K. (2011). Conceptual metaphors of emotion in spoken language: Good is up in semantics and prosody (Unpublished master's thesis). Victoria University, Wellington, New Zealand.

Goldsworthy, M. R., Pitcher, J. B., \& Ridding, M. C. (2013). Neuroplastic modulation of inhibitory motor cortical networks by spaced theta burst stimulation protocols. Brain stimulation, 6(3), 340-345. doi: 10.1016/j.brs.2012.06.005

Gordon, M. S., \& Rosenblum, L. D. (2005). Effects of intrastimulus modality change on audiovisual time-to-arrival judgments. Perception \& Psychophysics, 67(4), 580-594. doi: 10.3758/BF03193516

Gray, R., \& Regan, D. (1998). Accuracy of estimating time to collision using binocular and monocular information. Vision Research, 38(4), 499-512. doi: 10.1016/S0042-6989(97)00230-7

Gray, R., \& Regan, D. (2004). The use of binocular time-to-contact information. Advances in psychology, 135, 303-325. doi: 10.1016/S0166-4115(04)80015-3

Gray, R., \& Thornton, I. M. (2001). Exploring the link between time to collision and representational momentum. Perception, 30(8), 1007-1022. doi: 10.1068/p3220

Grossman, E. D., Battelli, L., \& Pascual-Leone, A. (2005). Repetitive TMS over posterior STS disrupts perception of biological motion. Vision research, 45(22), 2847-2853. doi: 10.1016/j.visres.2005.05.027 
Hancock, P. A., \& Manster, M. P. (1997). Time-to-contact: More than tau alone. Ecological Psychology, 9(4), 265-297. doi: 10.1207/s15326969eco0904_2

Hein, G., \& Knight, R. T. (2008). Superior temporal sulcus —it's my area: or is it? Cognitive Neuroscience, Journal of, 20(12), 2125-2136. doi: 10.1162/jocn.2008.20148

Heron, J., Whitaker, D., \& McGraw, P. V. (2004). Sensory uncertainty governs the extent of audio-visual interaction. Vision research, 44(25), 2875-2884. doi: 10.1016/j.visres.2004.07.001

Herwig, U., Satrapi, P., \& Schönfeldt-Lecuona, C. (2003). Using the international 10-20 EEG system for positioning of transcranial magnetic stimulation. Brain Topography, 16(2), 95-99. doi:10.1023/B:BRAT.0000006333.93597.9d

Hidaka, S., Teramoto, W., Keetels, M., \& Vroomen, J. (2013). Effect of pitch-space correspondence on sound-induced visual motion perception. Experimental brain research, 231(1), 117-126. doi: 10.1007/s00221-013-3674-2

Hikosaka, K., Iwai, E., \& Saito, H. (1988) Polysensory properties of neurons in the anterior bank of the caudal superior temporal sulcus of the macaque monkey. Journal of neurophysiology, 60(5), 1615-1637.

Hocking, J., \& Price, C. J. (2008). The role of the posterior superior temporal sulcus in audiovisual processing. Cerebral Cortex, 18(10), 2439-2449. doi: 10.1093/cercor/bhn007

Hofbauer, M., Wuerger, S. M., Meyer, G. F., Roehrbein, F., Schill, K., \& Zetzsche, C. (2004). Catching audiovisual mice: predicting the arrival time of auditory-visual motion signals. Cognitive, Affective, \& Behavioral Neuroscience, 4(2), 241-250. doi: 10.3758/CABN.4.2.241

Hubbard, T. L. (1997). Target size and displacement along the axis of implied gravitational attraction: Effects of implied weight and evidence of representational gravity. Journal of Experimental Psychology: Learning, Memory, and Cognition, 23(6), 1484-1493. doi: 10.1037/0278-7393.23.6.1484

Huang, Y. Z., Edwards, M. J., Rounis, E., Bhatia, K. P., \& Rothwell, J. C. (2005). Theta burst stimulation of the human motor cortex. Neuron, 45(2), 201-206. doi: 10.1016/j.neuron.2004.12.033

Hubbard, T. L., \& Courtney, J. R. (2009). Cross-modal influences on representational momentum and representational gravity. Proceedings of Fechner Day, 25(1), 173-178. doi: 10.1068/p6538 
Jacobs, R. A. (2002). What determines visual cue reliability? Trends in cognitive sciences, 6(8), 345-350. doi: 10.1016/S1364-6613(02)01948-4

James, T. W., VanDerKlok, R. M., Stevenson, R. A., \& James, K. H. (2011). Multisensory perception of action in posterior temporal and parietal cortices. Neuropsychologia, 49(1), 108-114. doi:

10.1016/j.neuropsychologia.2010.10.030

Julkunen, P., Säisänen, L., Danner, N., Niskanen, E., Hukkanen, T., Mervaala, E., \& Könönen, M. (2009). Comparison of navigated and non-navigated transcranial magnetic stimulation for motor cortex mapping, motor threshold and motor evoked potentials. Neuroimage, 44(3), 790-795. doi:

10.1016/j.neuroimage.2008.09.040

Kaas, A., Weigelt, S., Roebroeck, A., Kohler, A., \& Muckli, L. (2010). Imagery of a moving object: the role of occipital cortex and human MT/V5+. Neuroimage, 49(1), 794-804. doi: 10.1016/j.neuroimage.2009.07.055

Körding, K. P., Beierholm, U., Ma, W. J., Quartz, S., Tenenbaum, J. B., \& Shams, L. (2007). Causal inference in multisensory perception. PLoS one, 2(9), 1-10. doi: 10.1371/journal.pone.0000943

Laakso, I., Hirata, A., \& Ugawa, Y. (2014). Effects of coil orientation on the electric field induced by TMS over the hand motor area. Physics in medicine and biology, 59(1), 203-218. doi: 10.1088/0031-9155/59/1/203

Lee, D. N. (1976). A theory of visual control of braking based on information about time to collision. Perception, 5, 437-459. doi: 10.1068/p050437

Liebenthal, E., Desai, R. H., Humphries, C., Sabri, M., \& Desai, A. (2014). The functional organization of the left STS: a large scale meta-analysis of PET and fMRI studies of healthy adults. Frontiers in neuroscience, 8, 1-10. doi: 10.3389/fnins.2014.00289

Lidji, P., Kolinsky, R., Lochy, A., \& Morais, J. (2007). Spatial associations for musical stimuli: a piano in the head? Journal of Experimental Psychology: Human Perception and Performance, 33(5), 1189-1207. doi: 10.1037/00961523.33.5.1189

Logothetis, N. K., \& Pfeuffer, J. (2004). On the nature of the BOLD fMRI contrast mechanism. Magnetic resonance imaging, 22(10), 1517-1531. doi: 10.1016/j.mri.2004.10.018 
Maeda, F., Kanai, R., \& Shimojo, S. (2004). Changing pitch induced visual motion illusion. Current Biology, 14(23), 990-991. doi: 10.1016/j.cub.2004.11.018

Makin, A. D., Poliakoff, E., Chen, J., \& Stewart, A. J. (2008). The effect of previously viewed velocities on motion extrapolation. Vision research, 48(18), 1884-1893. doi: 10.1016/j.visres.2008.05.023

Makin, A. D. J., \& Bertamini, M. (2014). Do different types of dynamic extrapolation rely on the same mechanism? Journal of Experimental Psychology: Human Perception and Performance, 40(4), 1566-1579. doi: 10.1037/a0036680

Manabe, K., \& Riquimaroux, H. (2000). Sound controls velocity perception of visual apparent motion. The Journal of the Acoustical Society of Japan (E), 21(3), 171174. doi: 10.1250/ast.21.171

McBeath, M. K., \& Neuhoff, J. G. (2002). The Doppler effect is not what you think it is: Dramatic pitch change due to dynamic intensity change. Psychonomic bulletin \& review, 9(2), 306-313. doi: 10.3758/BF03196286

McGurk, H., \& MacDonald, J. (1976). Hearing lips and seeing voices. Nature, 264, 746-748. doi: 10.1038/264746a0

McIntyre, J., Zago, M., Berthoz, A., \& Lacquaniti, F. (2001). Does the brain model Newton's laws? Nature neuroscience, 4(7), 693-694. doi: 10.1038/89477

Murakami, T., Kell, C. A., Restle, J., Ugawa, Y., \& Ziemann, U. (2015). Left dorsal speech stream components and their contribution to phonological processing. The Journal of Neuroscience, 35(4), 1411-1422. doi:

10.1523/JNEUROSCI.0246-14.2015

Nagai, M., Kazai, K., \& Yagi, A. (2002). Larger forward memory displacement in the direction of gravity. Visual Cognition, 9(1-2), 28-40. doi: $10.1080 / 13506280143000304$

Neuhoff, J. G., \& McBeath, M. K. (1996). The Doppler illusion: the influence of dynamic intensity change on perceived pitch. Journal of Experimental Psychology: Human Perception and Performance, 22(4), 970-985. doi: 10.1037/0096-1523.22.4.970

Noesselt, T., Bergmann, D., Heinze, H. J., Münte, T., \& Spence, C. (2012). Coding of multisensory temporal patterns in human superior temporal sulcus. Frontiers in integrative neuroscience, 6, 1-14. doi: 10.3389/fnint.2012.00064

Noesselt, T., Rieger, J. W., Schoenfeld, M. A., Kanowski, M., Hinrichs, H., Heinze, H. J., \& Driver, J. (2007). Audiovisual temporal correspondence modulates human 
multisensory superior temporal sulcus plus primary sensory cortices. The Journal of neuroscience, 27(42), 11431-11441. doi:

10.1523/JNEUROSCI.2252-07.2007

Oberman, L., Edwards, D., Eldaief, M., \& Pascual-Leone, A. (2011). Safety of theta burst transcranial magnetic stimulation: a systematic review of the literature. Journal of Clinical Neurophysiology, 28(1), 67-86. doi: 10.1097/WNP.0b013e318205135f

Pascual-Leone, A., Walsh, V., \& Rothwell, J. (2000). Transcranial magnetic stimulation in cognitive neuroscience-virtual lesion, chronometry, and functional connectivity. Current opinion in neurobiology, 10(2), 232-237. doi: 10.1016/S0959-4388(00)00081-7

Peirce, J. W. (2007). PsychoPy—psychophysics software in Python. Journal of neuroscience methods, 162(1), 8-13. doi: 10.1016/j.jneumeth.2006.11.017

Peterken, C., Brown, B., \& Bowman, K. (1991). Predicting the future position of a moving target. Perception, 20, 5-16. doi: 10.1068/p200005

Poldrack, R. A. (2006). Can cognitive processes be inferred from neuroimaging data? Trends in cognitive sciences, 10(2), 59-63. doi: 10.1016/j.tics.2005.12.004

Prime, S. L., \& Harris, L. R. (2010). Predicting the position of moving audiovisual stimuli. Experimental brain research, 203(2), 249-260. doi: 10.1007/s00221010-2224-4

Radeau, M. (1994). Auditory-visual spatial interaction and modularity. Cahiers de Psychologie Cognitive/Current Psychology of Cognition, 13(1), 3-51.

Rahnev, D., Kok, P., Munneke, M., Bahdo, L., de Lange, F. P., \& Lau, H. (2013). Continuous theta burst transcranial magnetic stimulation reduces resting state connectivity between visual areas. Journal of neurophysiology, 110(8), 18111821. doi: 10.1152/jn.00209.2013.

Reynolds Jr, H. N. (1968). Temporal estimation in the perception of occluded motion. Perceptual and motor skills, 26(2), 407-416. doi: 10.2466/pms.1968.26.2.407

Roach, N. W., Heron, J., \& McGraw, P. V. (2006). Resolving multisensory conflict: a strategy for balancing the costs and benefits of audio-visual integration. Proceedings of the Royal Society B: biological sciences, 273, 2159-2168. doi : 10.1098/rspb.2006.3578 
Rusconi, E., Kwan, B., Giordano, B. L., Umilta, C., \& Butterworth, B. (2006). Spatial representation of pitch height: the SMARC effect. Cognition, 99(2), 113-129. doi: 10.1016/j.cognition.2005.01.004

Sack, A. T., Kadosh, R. C., Schuhmann, T., Moerel, M., Walsh, V., \& Goebel, R. (2009). Optimizing functional accuracy of TMS in cognitive studies: a comparison of methods. Journal of Cognitive Neuroscience, 21(2), 207-221. doi: 10.1162/jocn.2009.21126

Saito, D. N., Okada, T., Morita, Y., Yonekura, Y., \& Sadato, N. (2003). Tactile-visual cross-modal shape matching: a functional MRI study. Cognitive Brain Research, 17(1), 14-25. doi: 10.1016/S0926-6410(03)00076-4

Sandrini, M., Umiltà, C., \& Rusconi, E. (2011). The use of transcranial magnetic stimulation in cognitive neuroscience: a new synthesis of methodological issues. Neuroscience \& Biobehavioral Reviews, 35(3), 516-536. doi: 10.1016/j.neubiorev.2010.06.005

Saygin, A. P. (2007). Superior temporal and premotor brain areas necessary for biological motion perception. Brain, 130(9), 2452-2461. doi: 10.1093/brain/awm162

Senot, P., Zago, M., Lacquaniti, F., \& McIntyre, J. (2005). Anticipating the effects of gravity when intercepting moving objects: differentiating up and down based on nonvisual cues. Journal of neurophysiology, 94(6), 4471-4480. doi: 10.1152/jn.00527.2005

Schiff, W., \& Oldak, R. (1990). Accuracy of judging time to arrival: effects of modality, trajectory, and gender. Journal of Experimental Psychology: Human Perception and Performance, 16(2), 303. doi: 10.1037/0096-1523.16.2.303

Schmiedchen, K., Freigang, C., Nitsche, I., \& Rübsamen, R. (2012). Crossmodal interactions and multisensory integration in the perception of audio-visual motion-A free-field study. Brain research, 1466, 99-111. doi: 10.1016/j.brainres.2012.05.015

Shams, L., Kamitani, Y., \& Shimojo, S. (2002). Visual illusion induced by sound. Cognitive Brain Research, 14(1), 147-152. doi: 10.1016/S0926-6410(02)000691

Sparing, R., Buelte, D., Meister, I. G., Pauš, T., \& Fink, G. R. (2008). Transcranial magnetic stimulation and the challenge of coil placement: a comparison of 
conventional and stereotaxic neuronavigational strategies. Human brain mapping, 29(1), 82-96. doi: 10.1002/hbm.20360

Stefanovic, B., Warnking, J. M., \& Pike, G. B. (2004). Hemodynamic and metabolic responses to neuronal inhibition. Neuroimage, 22(2), 771-778. doi: 10.1016/j.neuroimage.2004.01.036

Stevens, S. S., Volkmann, J., \& Newman, E. B. (1937). A scale for the measurement of the psychological magnitude pitch. The Journal of the Acoustical Society of America, 8(3), 185-190. doi: 10.1121/1.1915893

Stevenson, R. A., \& James, T. W. (2009). Audiovisual integration in human superior temporal sulcus: Inverse effectiveness and the neural processing of speech and object recognition. Neuroimage, 44(3), 1210-1223. doi:

10.1016/j.neuroimage.2008.09.034

Stevenson, R. A., Mullin, C. R., Wallace, M. T., \& Steeves, J. K. (2013). fMRI-guided TMS of the superior temporal sulcus impairs multisensory temporal processing. Multisensory Research, 26, 208-208. doi: 10.1163/22134808-000S0156

Takeshima, Y., \& Gyoba, J. (2013). Changing Pitch of Sounds Alters Perceived Visual Motion Trajectory. Multisensory research, 26(4), 317-332. doi: $10.1163 / 22134808-00002422$

Talairach, J., \& Tournoux, P. (1988). Co-planar stereotaxic atlas of the human brain. 3Dimensional proportional system: an approach to cerebral imaging. Georg Thieme Verlag, Stuttgart/New York.

Tarnutzer, A. A., Lasker, A. G., \& Zee, D. S. (2013). Continuous theta-burst stimulation of the right superior temporal gyrus impairs self-motion perception. Experimental brain research, 230(3), 359-370. doi: 10.1007/s00221-013-3660-8

Teramoto, W., Hidaka, S., Gyoba, J., \& Suzuki, Y. (2010). Auditory temporal cues can modulate visual representational momentum. Attention, Perception, \& Psychophysics, 72(8), 2215-2226. doi: 10.3758/BF03196696

Thomson, R. H., Cleve, T. J., Bailey, N. W., Rogasch, N. C., Maller, J. J., Daskalakis, Z. J., \& Fitzgerald, P. B. (2013). Blood oxygenation changes modulated by coil orientation during prefrontal transcranial magnetic stimulation. Brain stimulation, 6(4), 576-581. doi: 10.1016/j.brs.2012.12.001 
Tranulis, C., Gueguen, B., Pham-Scottez, A., Vacheron, M. N., Cabelguen, G., Costantini, A., ... \& Galinovski, A. (2006). Motor threshold in transcranial magnetic stimulation: comparison of three estimation methods. Neurophysiologie Clinique/Clinical Neurophysiology, 36(1), 1-7. doi: 10.1016/j.neucli.2006.01.005

Tresilian, J. R. (1995). Perceptual and cognitive processes in time-to-contact estimation: analysis of prediction-motion and relative judgment tasks. Perception \& Psychophysics, 57(2), 231-245. doi: 10.3758/BF03206510

Tsang, P., Jacobs, M. F., Lee, K. G., Asmussen, M. J., Zapallow, C. M., \& Nelson, A. J. (2014). Continuous theta-burst stimulation over primary somatosensory cortex modulates short-latency afferent inhibition. Clinical Neurophysiology, 125(11), 2253-2259. doi: 10.1016/j.clinph.2014.02.026

VanVoorhis, C. W., \& Morgan, B. L. (2007). Understanding power and rules of thumb for determining sample sizes. Tutorials in Quantitative Methods for Psychology, 3(2), 43-50. doi :

van Atteveldt, N. M., Blau, V. C., Blomert, L., \& Goebel, R. (2010). fMR-adaptation indicates selectivity to audiovisual content congruency in distributed clusters in human superior temporal cortex. BMC neuroscience, 11(1), 11-22. doi: $10.1186 / 1471-2202-11-11$

van Kemenade, B. M., Muggleton, N., Walsh, V., \& Saygin, A. P. (2012). Effects of TMS over premotor and superior temporal cortices on biological motion perception. Journal of Cognitive Neuroscience, 24(4), 896-904. doi: 10.1162/jocn_a_00194

Wada, Y., Kitagawa, N., \& Noguchi, K. (2003). Audio-visual integration in temporal perception. International journal of psychophysiology, 50(1), 117-124. doi: 10.1016/S0167-8760(03)00128-4

Walker, B. N., \& Ehrenstein, A. (2000). Pitch and pitch change interact in auditory displays. Journal of Experimental Psychology: Applied, 6(1), 15-30. doi: 10.1037/1076-898X.6.1.15

Walsh, V., \& Cowey, A. (2000). Transcranial magnetic stimulation and cognitive neuroscience. Nature Reviews Neuroscience, 1(1), 73-80. doi: $10.1038 / 35036239$ 
Watanabe, K., \& Shimojo, S. (2001). When sound affects vision: effects of auditory grouping on visual motion perception. Psychological Science, 12(2), 109-116. doi:

Watson, R., Latinus, M., Noguchi, T., Garrod, O., Crabbe, F., \& Belin, P. (2014). Crossmodal Adaptation in Right Posterior Superior Temporal Sulcus during Face-Voice Emotional Integration. The Journal of Neuroscience, 34(20), 68136821. doi: 10.1523/JNEUROSCI.4478-13.2014

Welch, R. B., \& Warren, D. H. (1980). Immediate perceptual response to intersensory discrepancy. Psychological bulletin, 88(3), 638-667. doi: 10.1037/00332909.88.3.638

Werner, S., \& Noppeney, U. (2009). Superadditive responses in superior temporal sulcus predict audiovisual benefits in object categorization. Cerebral Cortex, 1829-1842. doi: 10.1093/cercor/bhp248

Westin, G. G., Bassi, B. D., Lisanby, S. H., Luber, B., Institute, N. Y. S. P., \& NY, U. (2014). Determination of motor threshold using visual observation overestimates transcranial magnetic stimulation dosage: Safety implications. Clinical Neurophysiology, 125(1), 142-147. doi: 10.1016/j.clinph.2013.06.187

Wu, S. W., Shahana, N., Huddleston, D. A., \& Gilbert, D. L. (2012). Effects of 30Hz Theta Burst Transcranial Magnetic Stimulation on the primary motor cortex. Journal of neuroscience methods, 208(2), 161-164. doi: 10.1016/j.jneumeth.2012.05.014

Wuerger, S., Meyer, G., Hofbauer, M., Zetzsche, C., \& Schill, K. (2010). Motion extrapolation of auditory-visual targets. Information Fusion, 11(1), 45-50. doi: 10.1016/j.inffus.2009.04.005

Zago, M., Iosa, M., Maffei, V., \& Lacquaniti, F. (2010). Extrapolation of vertical target motion through a brief visual occlusion. Experimental brain research, 201(3), 365-384. doi: 10.1007/s00221-009-2041-9

Zago, M., \& Lacquaniti, F. (2005a). Internal model of gravity for hand interception: parametric adaptation to zero-gravity visual targets on Earth. Journal of neurophysiology, 94(2), 1346-1357. doi: 10.1152/jn.00215.2005

Zago, M., \& Lacquaniti, F. (2005b). Visual perception and interception of falling objects: a review of evidence for an internal model of gravity. Journal of Neural Engineering, 2(3), 198-208. doi: 10.1088/1741-2560/2/3/S04 
Zago, M., McIntyre, J., Senot, P., \& Lacquaniti, F. (2008). Internal models and prediction of visual gravitational motion. Vision research, 48(14), 1532-1538. doi: 10.1016/j.visres.2008.04.005 


\section{Appendix A: Mean (SD) TTC Deviation for Experiment 1A}

Table A1.

Mean (SD) TTC deviation (s) for visual stimuli by direction, pitch, speed and landmark ( $L M)$ for Experiment 1A: Gradual Pitch Change at Occluder iin the horizontal plane.

TTC Deviation for Leftward Moving Stimuli

\begin{tabular}{cccccccccc} 
& \multicolumn{3}{c}{ Slow Stimulus Speed } & \multicolumn{2}{c}{ Medium Stimulus Speed } & \multicolumn{2}{c}{ Fast Stimulus Speed } \\
\multirow{2}{*}{ Pitch } & Near & Mid & Far LM & Near & Mid & Far LM & Near & Mid & Far LM \\
Condition & LM & LM & $M(S D)$ & LM & LM & $M(S D)$ & LM & LM & $M(S D)$ \\
& $M(S D)$ & $M(S D)$ & & $M(S D)$ & $M(S D)$ & & $M(S D)$ & $M(S D)$ & \\
\hline Constant & 0.33 & 0.28 & 0.18 & 0.34 & 0.37 & 0.39 & 0.31 & 0.42 & 0.44 \\
& $(0.20)$ & $(0.34)$ & $(0.48)$ & $(0.16)$ & $(0.26)$ & $(0.40)$ & $(0.16)$ & $(0.24)$ & $(0.31)$ \\
Rising & 0.33 & 0.23 & 0.06 & 0.35 & 0.38 & 0.33 & 0.35 & 0.42 & 0.44 \\
& $(0.25)$ & $(0.35)$ & $(0.48)$ & $(0.21)$ & $(0.28)$ & $(0.32)$ & $(0.21)$ & $(0.27)$ & $(0.35)$ \\
Falling & 0.36 & 0.25 & 0.17 & 0.38 & 0.42 & 0.43 & 0.36 & 0.44 & 0.54 \\
& $(0.21)$ & $(0.32)$ & $(0.39)$ & $(0.18)$ & $(0.28)$ & $(0.37)$ & $(0.20)$ & $(0.29)$ & $(0.36)$ \\
Silent & 0.31 & 0.29 & 0.16 & 0.33 & 0.34 & 0.34 & 0.30 & 0.35 & 0.43 \\
& $(0.21)$ & $(0.35)$ & $(0.47)$ & $(0.19)$ & $(0.27)$ & $(0.36)$ & $(0.15)$ & $(0.23)$ & $(0.31)$
\end{tabular}

TTC Deviation for Rightward Moving Stimuli

Slow Stimulus Speed $\quad$ Medium Stimulus Speed $\quad$ Fast Stimulus Speed

\begin{tabular}{lccccccccc}
\multirow{2}{*}{$\begin{array}{c}\text { Pitch } \\
\text { Condition }\end{array}$} & Near & Mid & Far LM & Near & Mid & Far LM & Near & Mid & Far LM \\
& $M(S D)$ & $M(S D)$ & & $M(S D)$ & $M(S D)$ & & $M(S D)$ & $M(S D)$ & \\
\hline Constant & 0.32 & 0.27 & 0.18 & 0.34 & 0.39 & 0.38 & 0.32 & 0.41 & 0.38 \\
& $(0.22)$ & $(0.32)$ & $(0.49)$ & $(0.17)$ & $(0.31)$ & $(0.33)$ & $(0.18)$ & $(0.26)$ & $(0.29)$ \\
\multirow{4}{*}{ Rising } & 0.31 & 0.16 & 0.04 & 0.37 & 0.35 & 0.32 & 0.33 & 0.40 & 0.41 \\
& $(0.21)$ & $(0.31)$ & $(0.36)$ & $(0.18)$ & $(0.27)$ & $(0.31)$ & $(0.19)$ & $(0.25)$ & $(0.29)$ \\
Falling & 0.35 & 0.30 & 0.10 & 0.36 & 0.39 & 0.42 & 0.36 & 0.45 & 0.48 \\
& $(0.20)$ & $(0.31)$ & $(0.40)$ & $(0.18)$ & $(0.26)$ & $(0.35)$ & $(0.20)$ & $(0.28)$ & $(0.29)$ \\
Silent & 0.32 & 0.28 & 0.13 & 0.33 & 0.34 & 0.39 & 0.29 & 0.34 & 0.36 \\
& $(0.21)$ & $(0.33)$ & $(0.41)$ & $(0.18)$ & $(0.27)$ & $(0.34)$ & $(0.17)$ & $(0.21)$ & $(0.25)$ \\
\hline
\end{tabular}


Table A2.

Mean (SD) TTC deviation (s) for visual stimuli by direction, pitch, speed and landmark (LM) for

Experiment 1A: Gradual Pitch Change at Occluder in the vertical plane.

TTC Deviation for Upward Moving Stimuli

\begin{tabular}{|c|c|c|c|c|c|c|c|c|c|}
\hline \multirow[b]{2}{*}{ Pitch } & \multicolumn{3}{|c|}{ Slow Stimulus Speed } & \multicolumn{3}{|c|}{ Medium Stimulus Speed } & \multicolumn{3}{|c|}{ Fast Stimulus Speed } \\
\hline & Near & Mid & Far LM & Near & Mid & Far LM & Near & Mid & Far LM \\
\hline \multirow[t]{2}{*}{ Condition } & LM & LM & $M(S D)$ & LM & LM & $M(S D)$ & LM & LM & $M(S D)$ \\
\hline & $M(S D)$ & $M(S D)$ & & $M(S D)$ & $M(S D)$ & & $M(S D)$ & $M(S D)$ & \\
\hline \multirow[t]{2}{*}{$\begin{array}{l}\text { Constant } \\
\end{array}$} & 0.31 & 0.29 & 0.23 & 0.33 & 0.44 & 0.47 & 0.33 & 0.50 & 0.43 \\
\hline & $(0.23)$ & $(0.31)$ & $(0.41)$ & $(0.21)$ & $(0.27)$ & $(0.33)$ & $(0.19)$ & $(0.28)$ & $(0.33)$ \\
\hline \multirow[t]{2}{*}{ Rising } & 0.31 & 0.23 & 0.14 & 0.37 & 0.43 & 0.50 & 0.33 & 0.47 & 0.58 \\
\hline & $(0.25)$ & $(0.32)$ & $(0.49)$ & $(0.21)$ & $(0.26)$ & $(0.39)$ & $(0.17)$ & $(0.27)$ & $(0.38)$ \\
\hline \multirow[t]{2}{*}{ Falling } & 0.33 & 0.32 & 0.19 & 0.35 & 0.46 & 0.55 & 0.37 & 0.59 & 0.66 \\
\hline & $(0.22)$ & $(0.34)$ & $(0.40)$ & $(0.20)$ & $(0.24)$ & $(0.33)$ & $(0.20)$ & $(0.30)$ & $(0.36)$ \\
\hline \multirow[t]{2}{*}{ Silent } & 0.31 & 0.31 & 0.28 & 0.34 & 0.43 & 0.46 & 0.31 & 0.43 & 0.52 \\
\hline & $(0.20)$ & $(0.34)$ & $(0.42)$ & $(0.18)$ & $(0.28)$ & $(0.35)$ & $(0.16)$ & $(0.23)$ & $(0.34)$ \\
\hline
\end{tabular}

TTC Deviation for Downward Moving Stimuli

Slow Stimulus Speed $\quad$ Medium Stimulus Speed $\quad$ Fast Stimulus Speed

\begin{tabular}{lccccccccc} 
Pitch & Near & Mid & Far LM & Near & Mid & Far LM & Near & Mid & Far LM \\
Condition & LM & LM & $M(S D)$ & LM & LM & $M(S D)$ & LM & LM & $M(S D)$ \\
& $M(S D)$ & $M(S D)$ & & $M(S D)$ & $M(S D)$ & & $M(S D)$ & $M(S D)$ & \\
\hline Constant & 0.27 & 0.24 & 0.15 & 0.33 & 0.36 & 0.39 & 0.28 & 0.38 & 0.43 \\
& $(0.21)$ & $(0.38)$ & $(0.44)$ & $(0.19)$ & $(0.27)$ & $(0.35)$ & $(0.16)$ & $(0.29)$ & $(0.33)$ \\
\multirow{5}{*}{ Rising } & 0.28 & 0.13 & -0.05 & 0.32 & 0.34 & 0.32 & 0.33 & 0.42 & 0.43 \\
& $(0.22)$ & $(0.32)$ & $(0.46)$ & $(0.18)$ & $(0.26)$ & $(0.36)$ & $(0.22)$ & $(0.29)$ & $(0.34)$ \\
Falling & 0.33 & 0.30 & 0.09 & 0.35 & 0.43 & 0.44 & 0.30 & 0.43 & 0.47 \\
& $(0.22)$ & $(0.31)$ & $(0.39)$ & $(0.24)$ & $(0.29)$ & $(0.36)$ & $(0.20)$ & $(0.26)$ & $(0.29)$ \\
Silent & 0.31 & 0.24 & 0.24 & 0.29 & 0.34 & 0.37 & 0.27 & 0.30 & 0.36 \\
& $(0.33)$ & $(0.33)$ & $(0.45)$ & $(0.18)$ & $(0.26)$ & $(0.37)$ & $(0.16)$ & $(0.20)$ & $(0.29)$ \\
\hline
\end{tabular}




\section{Appendix B: Mean (SD) TTC Deviation for Experiment 1B}

Table B1.

Mean (SD) TTC deviation ( $s$ ) for visual stimuli by direction, pitch, speed and landmark (LM) for Experiment 1B: Sudden Pitch Change at Occluder in the horizontal plane.

TTC Deviation for Leftward Moving Stimuli

\begin{tabular}{cccccccccc} 
& \multicolumn{2}{c}{ Slow Stimulus Speed } & \multicolumn{2}{c}{ Medium Stimulus Speed } & \multicolumn{2}{c}{ Fast Stimulus Speed } \\
\multirow{2}{*}{ Pitch } & Near & Mid & Far LM & Near & Mid & Far LM & Near & Mid & Far LM \\
Condition & LM & LM & $M(S D)$ & LM & LM & $M(S D)$ & LM & LM & $M(S D)$ \\
& $M(S D)$ & $M(S D)$ & & $M(S D)$ & $M(S D)$ & & $M(S D)$ & $M(S D)$ & \\
\hline Constant & 0.29 & 0.28 & 0.24 & 0.36 & 0.41 & 0.38 & 0.30 & 0.41 & 0.48 \\
& $(0.27)$ & $(0.41)$ & $(0.59)$ & $(0.24)$ & $(0.33)$ & $(0.40)$ & $(0.17)$ & $(0.24)$ & $(0.34)$ \\
Rising & 0.37 & 0.28 & 0.21 & 0.40 & 0.45 & 0.41 & 0.33 & 0.45 & 0.45 \\
& $(0.26)$ & $(0.38)$ & $(0.50)$ & $(0.25)$ & $(0.33)$ & $(0.40)$ & $(0.20)$ & $(0.28)$ & $(0.34)$ \\
Falling & 0.40 & 0.36 & 0.20 & 0.37 & 0.42 & 0.44 & 0.36 & 0.48 & 0.45 \\
& $(0.26)$ & $(0.38)$ & $(0.65)$ & $(0.19)$ & $(0.35)$ & $(0.38)$ & $(0.22)$ & $(0.28)$ & $(0.38)$ \\
Silent & 0.31 & 0.29 & 0.12 & 0.31 & 0.39 & 0.40 & 0.30 & 0.36 & 0.39 \\
& $(0.25)$ & $(0.46)$ & $(0.54)$ & $(0.18)$ & $(0.30)$ & $(0.44)$ & $(0.17)$ & $(0.26)$ & $(0.34)$ \\
\hline
\end{tabular}

TTC Deviation for Rightward Moving Stimuli

\begin{tabular}{cccccccccc} 
& \multicolumn{2}{c}{ Slow Stimulus Speed } & \multicolumn{2}{c}{ Medium Stimulus Speed } & \multicolumn{2}{c}{ Fast Stimulus Speed } \\
\multirow{2}{*}{ Pitch } & Near & Mid & Far LM & Near & Mid & Far LM & Near & Mid & Far LM \\
Condition & LM & LM & $M(S D)$ & LM & LM & $M(S D)$ & LM & LM & $M(S D)$ \\
& $M(S D)$ & $M(S D)$ & & $M(S D)$ & $M(S D)$ & & $M(S D)$ & $M(S D)$ & \\
\hline Constant & 0.30 & 0.23 & 0.08 & 0.31 & 0.36 & 0.37 & 0.33 & 0.44 & 0.53 \\
& $(0.25)$ & $(0.48)$ & $(0.64)$ & $(0.17)$ & $(0.31)$ & $(0.50)$ & $(0.25)$ & $(0.31)$ & $(0.39)$ \\
\multirow{2}{*}{ Rising } & 0.37 & 0.26 & 0.07 & 0.40 & 0.37 & 0.37 & 0.33 & 0.37 & 0.39 \\
& $(0.25)$ & $(0.40)$ & $(0.62)$ & $(0.22)$ & $(0.33)$ & $(0.46)$ & $(0.18)$ & $(0.24)$ & $(0.41)$ \\
Falling & 0.32 & 0.23 & 0.12 & 0.39 & 0.38 & 0.33 & 0.37 & 0.47 & 0.41 \\
& $(0.23)$ & $(0.38)$ & $(0.58)$ & $(0.19)$ & $(0.32)$ & $(0.36)$ & $(0.28)$ & $(0.35)$ & $(0.32)$ \\
Silent & 0.27 & 0.24 & 0.14 & 0.35 & 0.31 & 0.29 & 0.31 & 0.40 & 0.43 \\
& $(0.26)$ & $(0.41)$ & $(0.57)$ & $(0.22)$ & $(0.33)$ & $(0.38)$ & $(0.23)$ & $(0.35)$ & $(0.41)$ \\
\hline
\end{tabular}


Table B2.

Mean (SD) TTC deviation (s) for visual stimuli by direction, pitch, speed and landmark (LM) for

Experiment 1B: Sudden Pitch Change at Occluder in the vertical plane.

TTC Deviation for Upward Moving Stimuli

\begin{tabular}{|c|c|c|c|c|c|c|c|c|c|}
\hline \multirow[b]{2}{*}{ Pitch } & \multicolumn{3}{|c|}{ Slow Stimulus Speed } & \multicolumn{3}{|c|}{ Medium Stimulus Speed } & \multicolumn{3}{|c|}{ Fast Stimulus Speed } \\
\hline & Near & Mid & Far LM & Near & Mid & Far LM & Near & Mid & Far LM \\
\hline \multirow[t]{2}{*}{ Condition } & $\mathrm{LM}$ & LM & $M(S D)$ & LM & LM & $M(S D)$ & $\mathrm{LM}$ & LM & $M(S D)$ \\
\hline & $M(S D)$ & $M(S D)$ & & $M(S D)$ & $M(S D)$ & & $M(S D)$ & $M(S D)$ & \\
\hline \multirow[t]{2}{*}{$\overline{\text { Constant }}$} & 0.26 & 0.22 & 0.16 & 0.29 & 0.37 & 0.44 & 0.33 & 0.44 & 0.40 \\
\hline & $(0.25)$ & $(0.42)$ & $(0.54)$ & $(0.24)$ & $(0.37)$ & $(0.56)$ & $(0.19)$ & $(0.33)$ & $(0.45)$ \\
\hline \multirow[t]{2}{*}{ Rising } & 0.30 & 0.24 & 0.13 & 0.36 & 0.42 & 0.45 & 0.31 & 0.45 & 0.46 \\
\hline & $(0.24)$ & $(0.43)$ & $(0.62)$ & $(0.21)$ & $(0.37)$ & $(0.43)$ & $(0.22)$ & $(0.34)$ & $(0.34)$ \\
\hline \multirow[t]{2}{*}{ Falling } & 0.31 & 0.29 & 0.11 & 0.39 & 0.42 & 0.43 & 0.36 & 0.42 & 0.50 \\
\hline & $(0.30)$ & $(0.39)$ & $(0.51)$ & $(0.26)$ & $(0.36)$ & $(0.51)$ & $(0.22)$ & $(0.31)$ & $(0.39)$ \\
\hline \multirow[t]{2}{*}{ Silent } & 0.28 & 0.31 & 0.17 & 0.30 & 0.40 & 0.43 & 0.27 & 0.38 & 0.42 \\
\hline & $(0.26)$ & $(0.42)$ & $(0.62)$ & $(0.22)$ & $(0.32)$ & $(0.40)$ & $(0.17)$ & $(0.30)$ & $(0.35)$ \\
\hline
\end{tabular}

TTC Deviation for Downward Moving Stimuli

Slow Stimulus Speed $\quad$ Medium Stimulus Speed $\quad$ Fast Stimulus Speed

\begin{tabular}{lccccccccc}
\multirow{1}{*}{$\begin{array}{c}\text { Pitch } \\
\text { Condition }\end{array}$} & Near & Mid & Far LM & Near & Mid & Far LM & Near & Mid & Far LM \\
& $M(S D)$ & $M(S D)$ & & $M(S D)$ & $M(S D)$ & & $M(S D)$ & $M(S D)$ & \\
\hline Constant & 0.24 & 0.13 & 0.08 & 0.24 & 0.30 & 0.31 & 0.25 & 0.30 & 0.36 \\
& $(0.27)$ & $(0.45)$ & $(0.63)$ & $(0.21)$ & $(0.38)$ & $(0.46)$ & $(0.18)$ & $(0.30)$ & $(0.46)$ \\
\multirow{4}{*}{ Rising } & 0.28 & 0.21 & 0.05 & 0.34 & 0.38 & 0.30 & 0.29 & 0.37 & 0.34 \\
& $(0.24)$ & $(0.40)$ & $(0.56)$ & $(0.28)$ & $(0.36)$ & $(0.44)$ & $(0.21)$ & $(0.33)$ & $(0.38)$ \\
Falling & 0.28 & 0.26 & 0.09 & 0.34 & 0.34 & 0.34 & 0.26 & 0.40 & 0.36 \\
& $(0.29)$ & $(0.49)$ & $(0.58)$ & $(0.25)$ & $(0.35)$ & $(0.46)$ & $(0.18)$ & $(0.34)$ & $(0.39)$ \\
Silent & 0.26 & 0.18 & 0.09 & 0.28 & 0.29 & 0.32 & 0.20 & 0.20 & 0.29 \\
& $(0.29)$ & $(0.43)$ & $(0.60)$ & $(0.24)$ & $(0.33)$ & $(0.51)$ & $(0.17)$ & $(0.26)$ & $(0.40)$ \\
\hline
\end{tabular}




\section{Appendix C: Mean (SD) TTC Deviation for Experiment 2}

Table C1

Mean (SD) TTC deviation (s) for visual stimuli by direction, pitch, and TMS condition for Experiment 2 : Influence of TMS to the STS on Predicted Motion.

\section{TTC Deviation for Upward Moving Stimuli}

\begin{tabular}{lcccc} 
Pitch & Left STS & Right STS & Vertex & No TMS \\
Condition & $M(S D)$ & $M(S D)$ & $M(S D)$ & $M(S D)$ \\
\hline Constant & $0.61(0.56)$ & $0.59(0.42)$ & $0.50(0.57)$ & $0.53(0.46)$ \\
Rising & $0.49(0.54)$ & $0.51(0.43)$ & $0.42(0.54)$ & $0.41(0.48)$ \\
Falling & $0.74(0.52)$ & $0.63(0.38)$ & $0.63(0.54)$ & $0.62(0.45)$ \\
Silent & $0.71(0.58)$ & $0.68(0.48)$ & $0.57(0.58)$ & $0.58(0.56)$ \\
& & & & \\
\hline
\end{tabular}

TTC Deviation for Downward Moving Stimuli

\begin{tabular}{lcccc} 
Pitch & Left STS & Right STS & Vertex & No TMS \\
Condition & $M(S D)$ & $M(S D)$ & $M(S D)$ & $M(S D)$ \\
\hline Constant & $0.55(0.52)$ & $0.54(0.41)$ & $0.42(0.53)$ & $0.59(0.49)$ \\
Rising & $0.44(0.54)$ & $0.42(0.44)$ & $0.33(0.57)$ & $0.36(0.53)$ \\
Falling & $0.65(0.51)$ & $0.61(0.43)$ & $0.52(0.55)$ & $0.61(0.46)$ \\
Silent & $0.57(0.54)$ & $0.57(0.47)$ & $0.46(0.59)$ & $0.57(0.56)$ \\
& & & & \\
\hline
\end{tabular}

